$L A--9408-T$

DE82 019717

\title{
Multiplicity and Correlated Energy of Gamma Rays Emitted in the Spontaneous Fission of Californium-252
}

Glenn Samuel Brunson, Jr.*

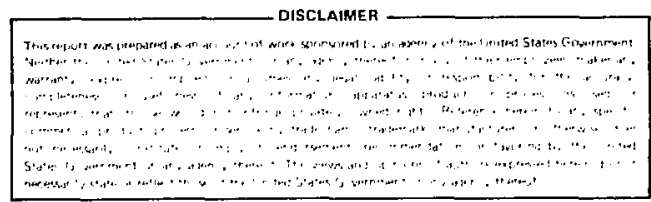

-Visiting Staff Member. 616 Bajada, Los Alamos, NM 67544.

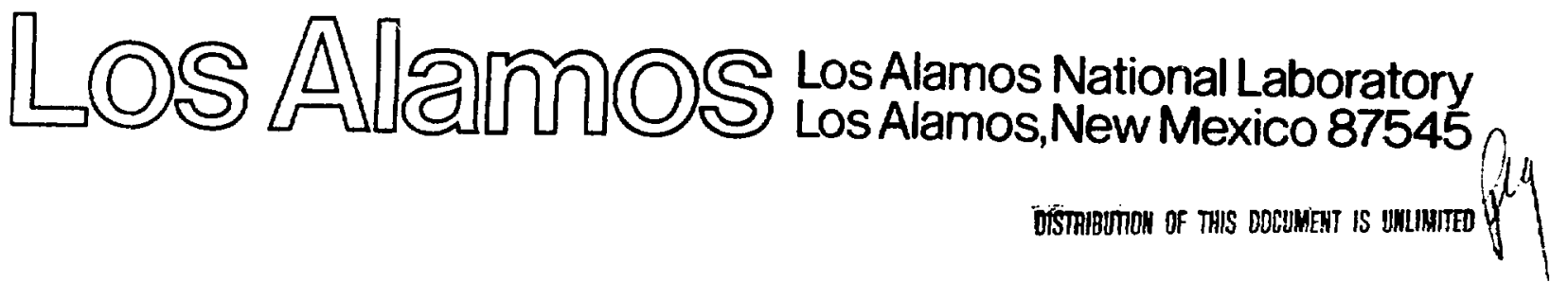




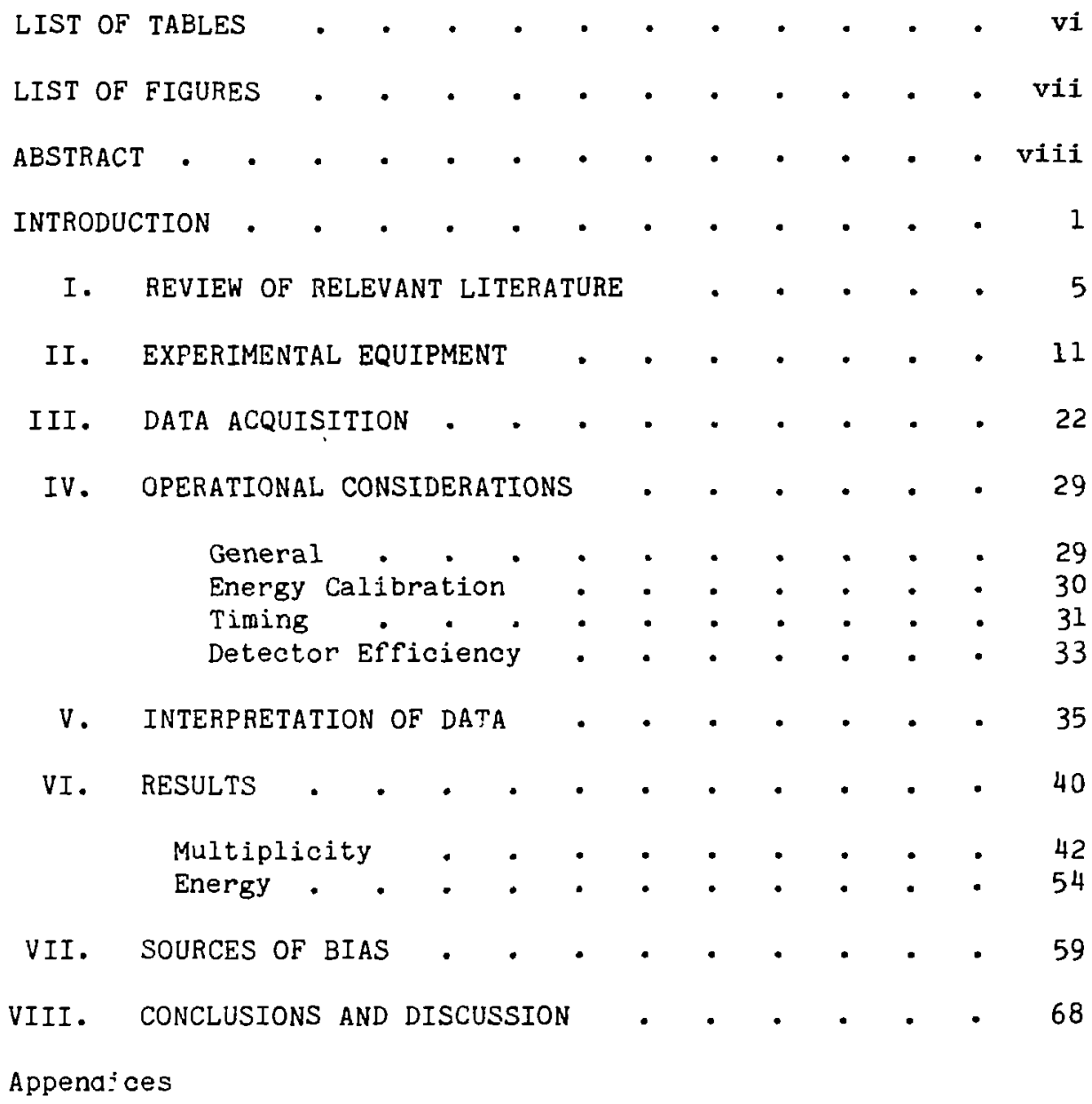

A. POSSIBLE MODELS FOR GAMMA EMISSION DISTRIBUTION $\bullet \quad \cdot \quad \bullet \quad \bullet \quad \bullet \quad \cdot \quad \cdot 1$

B. VAN DER WERF'S EQUATION • • • • • • • 75

C. FLOW CHARTS AND PROGRAM LISTINGS FOR THE

DATA ACQUISITION PROGRAM . . . . . . • . 78

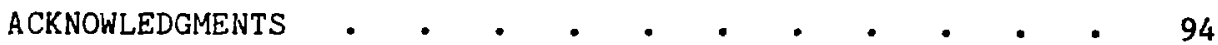
LIST OF REFERENCES • • • • • • • • • • • • 95 


\section{LIST OF TABLES}

1. Summary of available data on average number of gammas emitted in the spontaneous fission of ${ }^{252} \mathrm{Cf}$. . . . . 6

2. Raw data shown with correction for false counts and resulting corrected data . . . . . . . . . 41

3. Results obtained by fitting the double poisson model to corrected data (from Table 2) . . . . . . 48

4. Determination of average gamma energy associated with most prcbable emission number $\bar{G}(M)$. . . . . . . 55

5. Determination of false count probability . . . • • • 65 
1. Graphical summary of available data on the average number of gammas emitted in the spontaneous fission of ${ }^{252} \mathrm{Cf}$.

2. Perspective of the gamma detector array . . - . . 12

3. Spectrum of pulse heights from the fission detector . $\quad 17$

4. Time distribution of pulses in a typical gamma detector . 17

5. Schematic of the experimental set-up . . . . . . 19

6. Typica! output from data acquisition program . . . . 28

7. Comparison of stored spectra for various multiplicities - 28

8. Flow chart for the data analysis program . . . . . 38

9. Typical computer output from the data analysis program - 44

10. Experimental results for measurements at $140 \mathrm{keV}$ gamma threshold . . . . . . . . . . . . 45

11. Experimental results for measurements at $85 \mathrm{keV}$ gamma

12. Emission distribution inferred from the data in Figure 10 (140 keV threshold)

13. Emission distribution inferred from the data in Figure 11 (85 keV threshold) . . . . . . . . . 50

14. Comparison of emission distributions for Run $\$ 3$ as inferred from the double Poisson and skewed pseudo-Gaussian models . . . . . . . . .

15. Average energy of fission gammas as function of the most probable emission number . . . . . . . 56

16. Flow chart for the main data acquisition program • • . 79

17. Flow chart for the assembly language subroutine called by the main program to acquire data . . . . . . 80 


\section{ABSTRACT}

An array of eight high-speed plastic scintillation detectors has been used to infer a mathematical model for the emission multiplicity of prompt gammas in the spontaneous fission of ${ }^{252} \mathrm{Cf}$. Exceptional time resolution and coincidence capability permitted the separation of gammas from fast neutrons over a flight path of approximately $10 \mathrm{~cm}$. About 20 different distribution models were tested; the best (termed "double Poisson") was

$$
\left[\begin{array}{l}
\text { Prob of emitting } \\
\text { exactly } n \text { gammas }
\end{array}\right]=\frac{A(B)^{n} e^{-B}}{n !}+\frac{(1-A)(C)^{n} e^{-C}}{n !}, A<1 \text {. }
$$

For a gamma threshold of $140 \mathrm{kev}$, the model with parameters evaluated reads

$$
\pi(n)=0.675 \frac{(6.78)^{n}}{n !} e^{-6.78}+0.325 \frac{(9.92)^{n}}{n !} e^{-9.92} .
$$

The average energy of the prompt gammas is inversely related to the number emitted; however, this inverse relationship is not strong and the total gamma energy does increase with increasing gamma number.

An extension of the experiment incorporated a lithium-drifted germanium gamma spectrometer that resolved nearly 100 discrete gammas associated with fission. Of these gammas, some were preferentially associated with fission in which few gammas were emitted. Certain others were more frequent when many gammas were emitted. 
MULTIPLICITY AND CORRELATED ENERGY OF GAMMA RAYS EMITTED IN THE SPONTANEOUS FISSION OF CALIFORNIUM-252

by

Glenn Samuel Brunson, Jr.

INTRODUCTION

The general objective in this study of gama-ray multiplicity was to add to knowledge of a relatively unexplored aspect of the fission phenomenon. As will appear later, almost no work has been done previously toward determining the probability distribution of the number of gammas emitted in the spontaneous fission of ${ }^{252} \mathrm{Cf}$.

Measurement of gamma multiplicity is more complicated tnan tne measurement of reutron multiplicity although the two are similar. Neutron multiplicity measurements are customarily made at the center of a single large, hollow scintillation detector containing of the order of 500 liters of liquid scintillator tnat is viewed by a number of pnotomultipliers operated in parallel.[1] The scintillator is loaded witn a strong neutron absorber such as gadolinium. Prompt (fast) neutrons given of in fission are down-scattered in energy by the hydrogenous liquid until tney are lost from the system or captured by the gadolinium. The nign energy gammas from neutron capture are detected in the scintillator and recorded as neutron events.

The detector is gated off to ignore the "gamma flash" that occurs when the prompt fission gammas simultaneously strike the scintillator within about one nanosecond after fission. By contrast, the neutrons require several microseconds for thermalization and capture. This slowing down time effectively separates the neutron capture events from each otner. Thus, the neutron counter, with suitable gating, can 
ignore the prompt gammas and then count several (possibly all) neutrons from a given fission; the dispersion in capture times is large enough that deadtime corrections are small. A typical neutron counter for this purpose may achieve an efficiency of 0.80 or higher.

Counting fission gamas is quite different. Here there can be no time separation because all the prompt gammas arrive at the detector at virtually the same instant. Because there is no time separation to permit the counting of individual gammas, the gamma multiplicity experiment must depend on spatial separation. This means having a substantial number of independent gamma detectors. Geometrically we

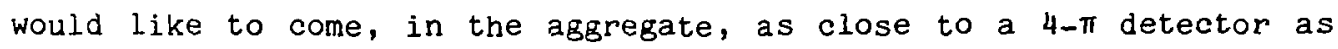
possible. Detecting an n-fold coincidence depends, in the first approximation, on the total counter sensitivity to the nth power.

In the case of the single neutron counter discussed above, it is actually possible to count all the neutrons at the highest credible multiplicity, say nine. It is not possible, however, to count all the gammas from a single fission without a quite large number of detectors. If two or more gammas from a fission strike the same detector, we will count them as a single gamma event.

Various values for the average number of gammas emitted in fission of ${ }^{252} \mathrm{Cf}$ have been reported in the literature.[2-6] The number ranges from approximately 7 to 10 . The literature on this point is reviewed in detail in a separate section. Here is an indication of the wide range of this work beyond the measurements of average neutron and gamma yields: 
1. Rumpold et al.[8] have been able to associate specific gamma energies with fission fragment energy and with estimated half-lives for the decay in question.

2. Val'skii et al.[4] in Russia have investigated the gammaray yield as a function of total fragment kinetic energy.

3. Lajtai et al.[9] in Hungary and Skarsvag[10] in Norway have studied the angular distribution of gammas from ${ }^{252} \mathrm{Cf}$ fission relative to the axis of separation of the two fission fragments.

4. Nifenecker et al.[11] in France have found a positive correlation between the number of neutrons emitted and the tolal gamma energy.

5. Ramamurthy et al.[12] in India have studied the multiplicity of prompt fission gammas from ${ }^{252} \mathrm{Cf}$. Only the Ramamurthy group seems to have studied the gamma multiplicity explicitly; their experiment is reviewed in the next section.

We have used eight high speed plastic scintillators and a coincidence criterion of about four nanoseconds. Because eight detectors cannot detect the maximum number of gammas that may be emitted in a fission, sur approach is simply to construct a mathematical model of the emission multiplicity distribution that is most consistent with the detection multiplicities actually recorded.

We suppose the model to have the following characteristics:

$$
\begin{gathered}
\text { 1. } \sum_{n=0}^{\infty} \pi(n)=1, \text { where } \pi(n)=\text { probability that a fission emits } \\
\text { exactly } n \text { gammas. }
\end{gathered}
$$


2. $\sum_{n=0}^{\infty} n \pi(n)=\bar{n}$, where $\bar{n}=$ average number of gammas emitted.

3. The distribution is nonnegative.

4. The distribution is in some sense smooth.

We seek an analytical expression for $\pi(n)$, the emission probability distribution, which can be reconciled most closely with $P(M)$, the detected multiplicity distribution. The criterion for judging the best model is a weighted least squares comparison with experimental data.

Once the counter array was constructed and operating, we were able to extend the investigation to the relationship between the number of gammas emitted and the average energy of those gammas.

The experiment depends critically on timing. Practical considerations led to an apparatus with eight gamma scintillation detectors $(127 \times 127 \mathrm{~mm})$ clustered as tightiy as possible around the source. This resulted in a source-to-detector distance of only $10 \mathrm{~cm}$. Over this flight path, gammas are separated from the most energetic fission neutrons by about three nanoseconds, hence experimental timing must achieve time definition that is a fraction of this minimum separation. 
REVIEW OF RELEVANT LITERATURE

There are five available measurements of $\bar{n}$, the average number of gammas emitted in the spontaneous fission of ${ }^{252} \mathrm{Cf}[2-6]$ and only one reference dealing with the characteristics of the emission probability distribution.[12] The measurements of $\bar{n}$ are summarized in Table 1 insofar as they can be determined from the references.

It has been customary to treat the average gamma yield $\bar{n}$ in the same fashion as $\bar{v}$, the average prompt neutron yield. That is, it is tacitly assumed that all gammas are, in principle, detectable just as all neutrons are detectable. In fact, $\bar{n}$ is clearly a function of the gamma detector threshold, although only verbinski et al.[5] explicitly recognized that fact. As an example of the usual treatment, Hoffman and Hoffman[7] make a statistical combination of the last three values in the table without noting that they refer to different thresholds. The dependence on threshold is strong, as seen in Table 1. Entries in the table have been drawn by inference where specific information was not available. For example, the threshold for the first entry (Smith)[2] was inferred approximately from the remark that a small photopeak was detected near $60 \mathrm{keV}$. The threshold for the second entry was inferred from the left-hand-most point in Figure 6 of Reference 3. Thresholds are given in References 4 and 5 . The threshold 
TABLE 1

SUMMARY OF AVAILABLE DATA ON AVERAGE NUMBER OF GAMMAS $(\bar{n})$ EMITTED IN SPONTANEOUS FISSION OF ${ }^{252} \mathrm{Cf}$

\begin{tabular}{|c|c|c|c|c|c|c|}
\hline Date & $\bar{n}$ & $\boldsymbol{\sigma}$ & $\begin{array}{l}\text { Threshold } \\
\text { (keV) }\end{array}$ & $\begin{array}{c}\overline{E^{a}} \\
(\mathrm{MeV})\end{array}$ & First Author & Ref. \\
\hline 1956 & 10.3 & $?$ & $\approx 40 ?$ & 0.80 & A. B. Smith & 2 \\
\hline 1958 & 10 & $?$ & 240? & 0.9 & $H \cdot R$. Bowman & 3 \\
\hline 1969 & 7.5 & 1.5 & 100 & & G. V. Val'skii & 4 \\
\hline 1973 & 7.8 & 0.3 & 140 & .0 .88 & Verbinsk: & 5 \\
\hline 1974 & 8.32 & 0.4 & 85 & 0.85 & F. Pleasonton & 6 \\
\hline
\end{tabular}

A Average energy per gamma. 
for the unpublished experiment by Pleasanton was obtained from the experimenter. It is important to note that all of these results were obtained using sodium iodide detectors.

Despite the fact that the first two measurements in Table 1 were mide well more than 20 years ago with relatively primitive equipment, they are in better agreement with later measurements than it at first appears. In Figure $1, \bar{n}$ is shown as a function of discriminator threshold. The values of $\bar{n}$ for some higher thresholds have been taken from Table I of the Verbinski paper and included in Figure 1 . There is an upward trend with decreasing threshold; this is to be expected as more gammas become "eligible" for cietection. Moreover, there is another phenomenon that becomes of interest in the region of $40 \mathrm{keV}$ and below. Glendenin et al.[13] have found $x$ rays in the region of $40 \mathrm{keV}$ that are associated with the heavier of the two fission fragments. Thus, when the threshold is lowered to $40 \mathrm{keV}$, some $x$ rays will be counted indistinguishably with low energy gammas; this phenomenon may have contributed to the larger values obtained for $\bar{n}$ in the earlier measurements.

As another point of comparison, Smilh et al.[2] estimated that there were 5.0 gammas per fission between 0.5 and $2.3 \mathrm{MeV}$. We can estimate from Table I of Reference 5 that Verbinski et al. found 4.1 gammas in the same energy interval. Also, we note the qualitative agreement between References 5 and 6 with respect to the average energy per fission gamma. Reference 6, with a lower threshold, reports a slightly lower average gamma energy, as it should. 


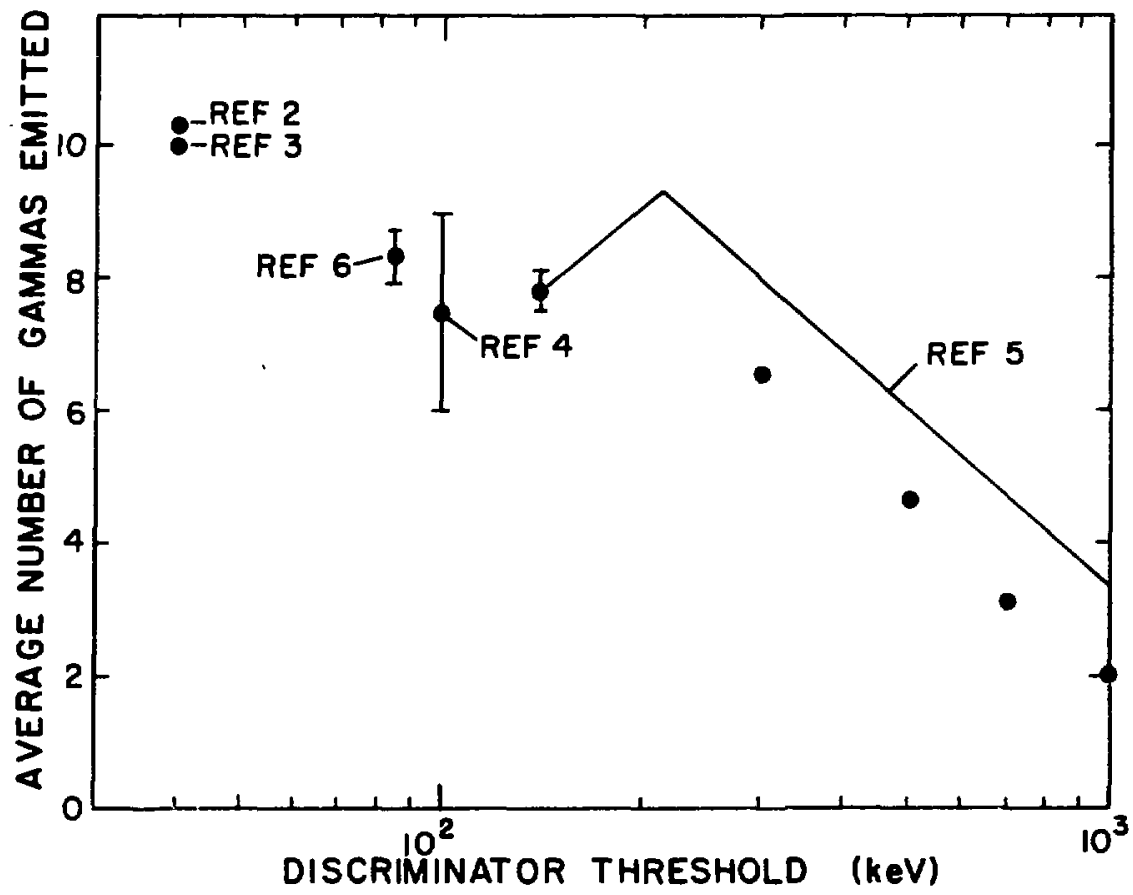

Fig. 1. Graphical summary of available data on the average number of prompt gammas emitted in the spontaneous fission of ${ }^{252} \mathrm{Cf}$. 
We turn now to the work of Ramamurthy et al.,[12] which appears to be the only previous attempt to characterize the emission distribution of gammas from the spontaneous fission of ${ }^{252} \mathrm{Cf}$. There are several problems with this experiment. The experimenters (in 1977) assumed $\bar{n}=10.3$, attributing this number to Johansson and Kleinheinz [14] when, in fact, these authors seem merely to have been citing the 1956 results of Smith et al.[2] This overlooks the last three entries in Table 1, all of which were available in 1977. They worked with only three gamma detectors, too few to obtain meaningful statistics when emission multiplicities may easily be 15 or greater.

No mention is made of the gamma threshold nor of the time criterion for coincidence. Mo mention is made of the threshold level of the inltiating fission detector. In the present work, this has been found to be a serious source of bias if not properly adjusted.

In analyzing the results, the Ramamurthy group did not take into account the complexity of the probabilities involved. For example, they write for the singles detection rate per fission in a fiven counter i

$$
c_{i}=\bar{n} \cdot \Omega_{i}
$$

where $\Omega_{i}$ is the detection efficiency and $\bar{n}$ is the average number of gammas emitted. This does not take into account tne fact that the probability of a single detection is actually a compound probability that includes the "miss" probabilities for the other detectors. These "miss" probabilities are not, in general, negligibly close to unity. Moreover, they are nonlinear $\left[\right.$ of the form $\left.\left(1-\Omega_{i}\right)^{n}\right]$ and, therefore, must be evaluated for the enission probability distribution. 
Ramamurthy and associates concluded that their data were consistent with a Gaussian gamma emission distribution having $\bar{n}=10.3$ and a standard deviation of $4.2 \pm 0.4$. 
EXPERIMENTAL EQUIPIIENT

The experimental gamma counter array consisled of eight large cylindrical scintillation detectors clustered symmetrically around the californium fission source (see Figure 2). The fission source was electro-deposited on a stainless steel disc and placed in intimate contact with a thin scintillator on a small photomultiplier. This fission detector provided a timing signal on the occurrence of a fission. Each such signal initiated (if the system was in the quiescent state) an analysis cycle that counted the coincident gammas in the surrounding eight detectors.

The scintillator on the fission detector was of Pilot U.[15] It was $0.25 \mathrm{~mm}$ thick by about $20 \mathrm{~mm}$ in diameter, and in intimate contact with the source foil that carries the ${ }^{252} \mathrm{Cf}$ deposit as a $6 \mathrm{~mm}$ circular spot at its center. The source strength was about 800 fissions per second. The other side of the scintillator was coupled to an RCA 4886 photomultiplier[16] (3/4 inch nominal diameter) with silicone optical grease. This tube has a photocathode about $13 \mathrm{~mm}$ in diameter. The fission fragments had flight paths on the order of microns and impinged on the scintillatcr ir a spot comparable in size to that of the active deposit. This means that most photoelectrons originated in a similarly sized spot on the photocathode, and the envelope of their trajectories 


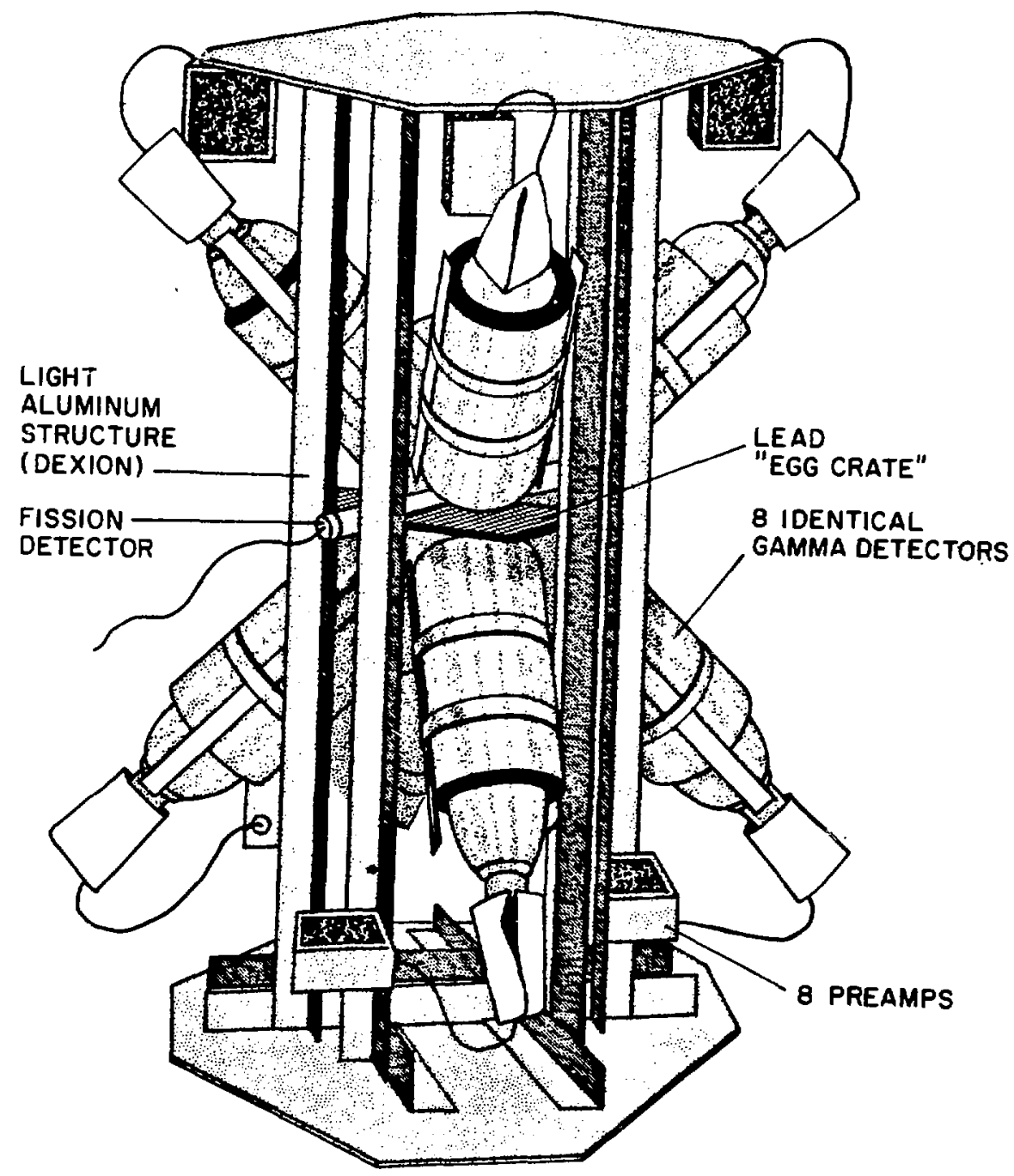

Fig. 2. Perspective of the gamna detector array. The small fission detector assembly extends so that the fissionable material is at the center of the counting geometry. 
to the first dynode was a narrow cone in which transit times did not differ much. The result was small time dispersion for the trigger pulses originating in the fission detector. To reduce the amount of scattering materi 1 near the source, the fission detector was operated without a mu-metal shield. For this reason, much care was required to assure that the fission detector was returned to the same rotational orientation each time it was removed. Otherwise, the varying effects of the earth's magnetic field could cause perceptible changes in the amplitude of the output. The fission detector was operated at -700 volts bias and the signal did not require amplification before input to the discriminator.

In ${ }^{252} \mathrm{Cf}$, the principal mode decay is by alpha enission ( $\left.97 \%\right)$. The other $3 \%$ is by spontaneous fission.[17] Taking into account two fission fragments per fission, the emission rate of alphas exceeds that of fission fragments by a factor of about 16. Both were detected by the scintillator. The alpha particles from ${ }^{252}$ Cf are emitted with energies up to $6.1 \mathrm{MeV}$; the fission fragments have energies in the range $70-100 \mathrm{MeV}$. Given the relatively poor energy resolution of plastic scintillators, care was necessary to set the discriminator so as to trigger on an average distribution of fission events. Figure 3 shows the spectrum of pulses occurring in the fission detector. The highest peak represents aipha events and the two lower humps correspond to the bimodal distribution of fission fragment energies. The right (high energy) hump corresponds to the lighter fission fragments because they receive the greater energy in the fission process. 
It is surprising to find the approximately $6 \mathrm{MeV}$ alpha particles so close to the approximately $70 \mathrm{MeV}$ heavy fission fragments; this is attributable to the differing response of the scintillator to the two distinct particle types. However, with the possible exception of the rare symmetric fission, every fission event must be represented by one fragment in each of the humps of the energy distribution. Thus, if the discriminator threshold is set exactly between the two humps, the experiment will be triggered on a represenative distribution of all fission events.

Suppose, on the other hand, the threshold is set relatively high so as to accept only the upper $10 \%$ of the pulses in the upper hump of the fission fragment energy distribution. This will bias the triggering events in two ways:

1. It will tend to select fissions in which the mass partition is highly asymmetric because these are the events that produce the most energetic light fission fragments.

2. And of these fissions, the selected events are further biased toward relatively lower excitation energies because low excitation leaves more kinetic energy available to interact with the scintillator.

The lower excitation energy is evidenced by reduced yield of prompt neutrons and total gamma energy. This effect has been measured by Nifenecker[11] and our results are in qualitative agreement with respect to the gammas.

The eight gamma detectors, $127 \times 127-\mathrm{mm}$ cylinders of the plastic scintillator NE102,[15] were mounted on Amperex XP2040[18] photomultipliers with silicone optical grease. The scintillators were wrapped 
dry with reflective aluminum foil. The photomultipliers were fitted with mu-metal shields. This photomultiplier has 14 stages and, when operated at -2250 volts bias voltage, did not require amplification before the signal was fed to the discriminator (Phillips 715).[19]

The gamma detectors were symmetrically supported around the fission source on light aluminum structural members (Dexion). Pairs of detectors faced each other along, and were coaxial with, each of the four diagonals of a cube. All were brought toward the center of the cube until their edges touched. The resulting enclosed space was conceptually a regular octahedron with about 200 mm between the faces of opposing scinillators. Fach of the scintillators was wrapped on its curved surface with 1.6 mo of lead sheet. In addition, there was an "egg crate" of 3.2-mm lead plates that formed a separate cell for each detector. This egg crate was cut away at the center of the counting geometry to provide space for the fissiun counter assembly. The purpose of the lead snielding was to minimize "cross talk" between detectors. It was anticipated (and observed) that, without shielding, a single gamma could, by a compton or other event, give a spurious coincidence between adjacent detectors. Subsequent measurements indicated that the lead described above substantially reduced the effect in adjacent detectors.

Clearly, if we were to discriminate between gamas and fast neutrons on a flight path of only 10 centimeters, timing was critical everywhere in the experiment. The fission counter as mentioned above contributed a relatively small amount to dispersion in timing. The gamma detectors did exhibit some dispersion from various causes. 
The photocathode of the XP2040 is about $110 \mathrm{~mm}$ in diameter and is spherical in form to equalize electron flight times to the first dynode. Nevertheless, there is a transit time difference of about one nanosecond between the center and the edge of the photocathode. Even though a given gamma event will create photoelectrons from many points of the photocathode, this transit time difference tends to vary the pulse shape and hence timing, depending on where in the scintillator the event occurred:

Another factor contributing to time dispersion in the gamma detectors is the velocity difference for visible light and gama rays in the scintillator material. A gamma ray can traverse the thickness of the $127 \mathrm{~mm}$ scintillator in about 0.42 nanoseconds while visible light required about 0.67 nanoseconds to travel the same distance. (The index of refraction is 1.58.)[15] Thus, everythini else remaining the same, there can be as much as $1 / 4$ nanosecond difference in the time of creation of the first photoelectron depending onl; on whether the gamma interaction with the scintillator occurred near the front face or near the photocathode. In addition, the shape of the signal pulse and hence the reaction time of the discriminator will also vary with the location of the scintillation event because of the varying paths the photons can follow before interacting with the photocathode.

The composite time resolution as measured with a time to pulse height converter from a fission counter trigger tc an associated fission gamma event in one of the gamma detectors was about 1.1 nanoseconds full width at half maximum (see Figure 4). The time scale was 


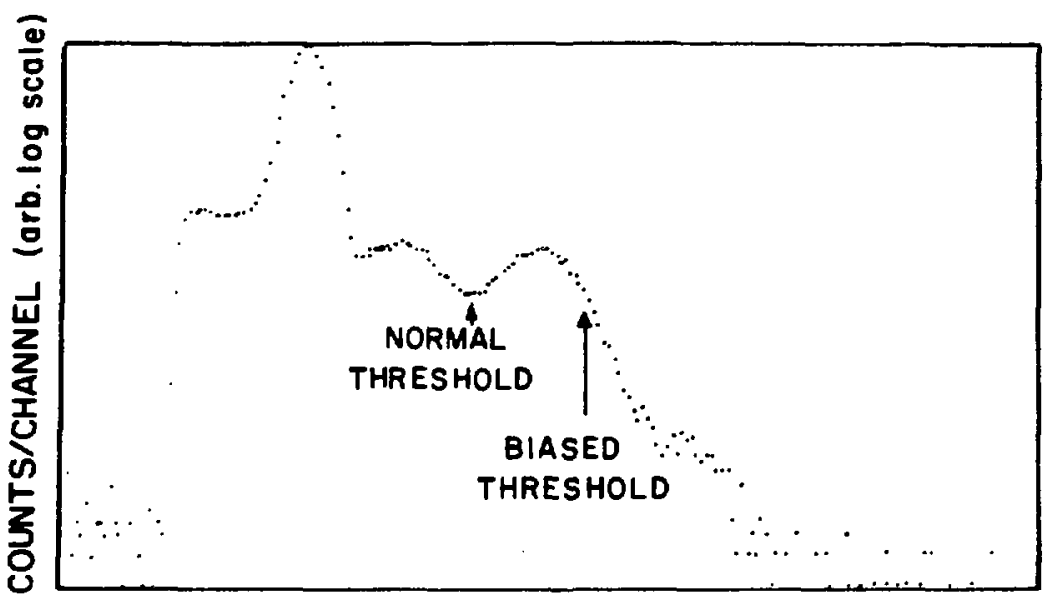

ENERGY (arb. scale)

Fig. 3. Spectrum of pulse heights from the fission detector. The arrows indicate the location of the two threshold pulse heights referred to in the text.

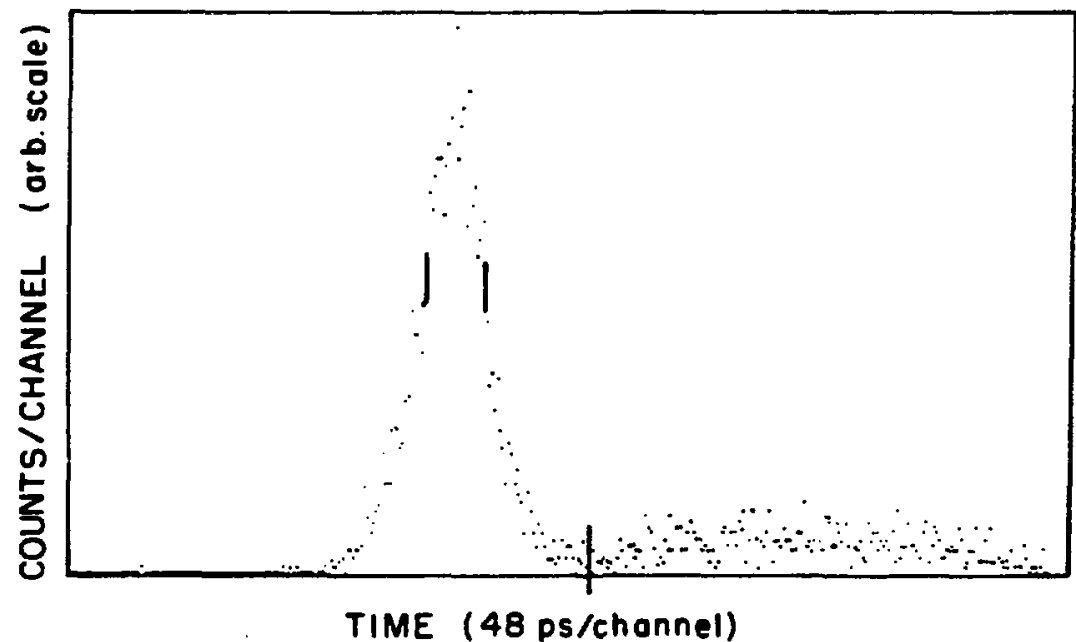

Fig. 4. Time distribution of pulses in a typical gamma detector. This was obtained by triggering a time-to-pulse height converter with a fission detector pulse and observing the arrival of gammas and fast neutrons at one of the eight gamma detectors. The upper two markers indicate a resolution of $1 \mathrm{nsec}$ at half maximum. The lower marker indicates the approximate point of partition between gammas and fast neutrons. 
approximately 48 picoseconds per channel and the markers on the peak are separated by approximately 1 nanosecond. The marker on the baseline indicates the approximate boundary between jaminas and fast neutrons.

The experiment depended critically on the characteristics of the discriminators. The Phillips Model 715 constant fraction discriminator contains five independent chamels in a single NIM module. The threshold has a range from -25 to $-1000 \mathrm{mV}$. The module also has a VETO input that is cummon to all five channels; this permits an external signal to turn off the channels while analysis of the last event is going on. These discriminators, due to their constant fraction characteristic, have exceptional timing performance. They are said to have less than 150 piccseconds "walk" (time shift) for inputs from $-25 \mathrm{mV}$ to $-2.5 \mathrm{~V}$. This is predicated on constant pulse shape, which as mentioned above, cannot be assumed under our operating conditions. The minimum width of the discriminator output is 5 nanoseconds and the rise and fall times are approximately 1 nanosecond. The minimum pulse pair separation is 9.5 nanoseconds if the output is at minimum width. There is also a 9.5-nanosecond delay between input and output. The output is $-800 \mathrm{mV}$ and compatible with system requirements for counting and gating.

Aside from the detectors and associated power supplied, the experimental equipment (schematic in Figure 5) consisted of standard nuclear electronic modules of two general types, NIM and CAMAC. NIM modules are plugged into a NIM "bin" and partake of a common power source, but all signal and data handling is accomplished externally by 


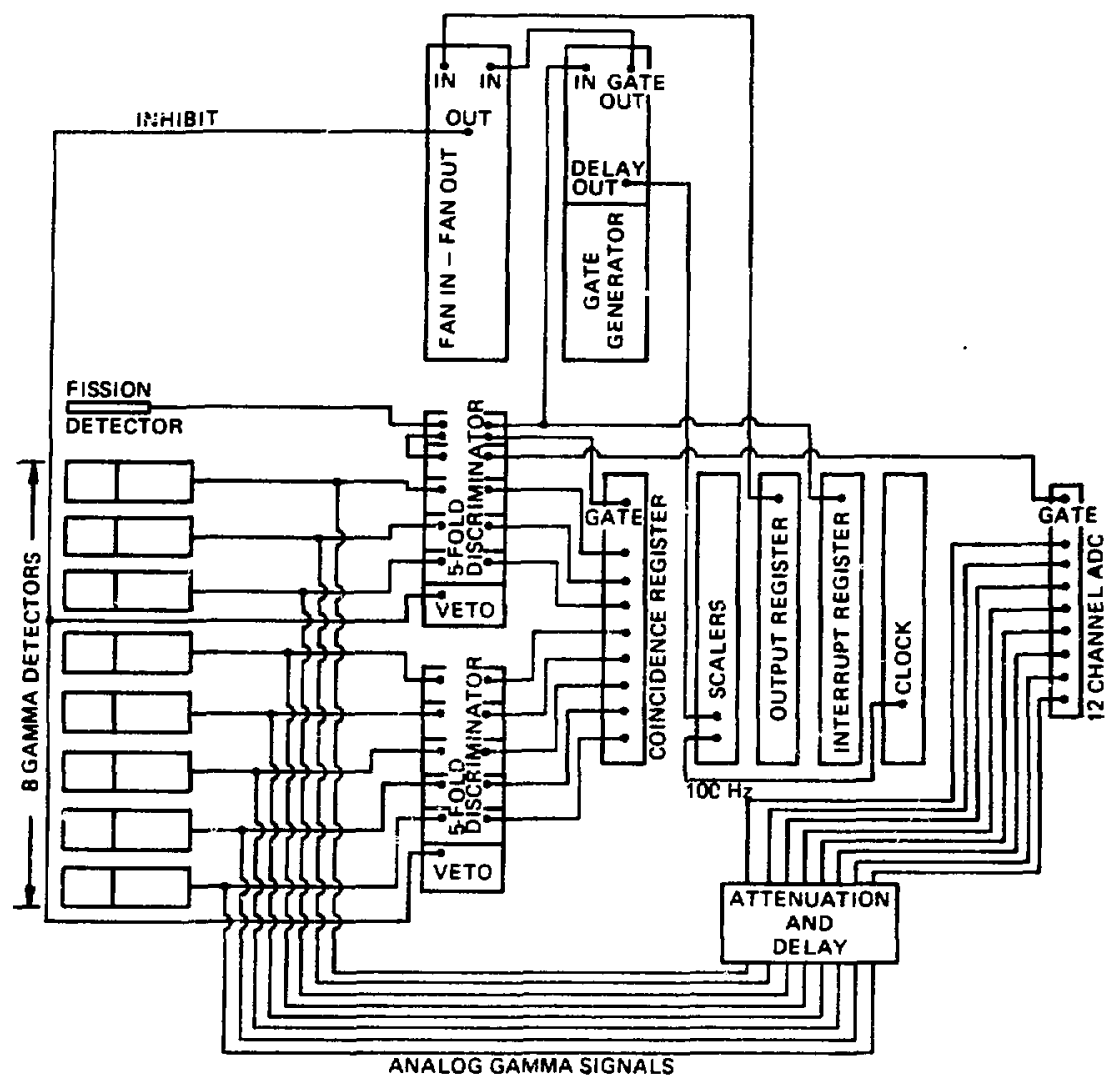

Fig. 5. Schematic of the experimental set-up. Identification of the instrument modules:

\begin{tabular}{|c|c|c|c|}
\hline \multirow{3}{*}{$\begin{array}{l}\text { NIM type } \\
\text { (wide boxes) }\end{array}$} & Function & \multirow{2}{*}{$\frac{\text { Maker }}{\text { Phillips }}$} & \multirow{2}{*}{$\frac{\text { Model No. }}{715}$} \\
\hline & $\begin{array}{l}\text { 5-fold Constant } \\
\text { Fraction Discriminator }\end{array}$ & & \\
\hline & $\begin{array}{l}\text { 2-fold Gate } \\
\text { Generator }\end{array}$ & Lecroy & 222 \\
\hline & Fan-in fan-out & Lecroy & 429 \\
\hline \multirow[t]{6}{*}{$\begin{array}{l}\text { CAMAC type } \\
\text { (narrow bo: }: s \text { ) }\end{array}$} & $\begin{array}{c}\text { 16-fold Coincidence } \\
\text { Register }\end{array}$ & Lecroy & $2 ? 41 \mathrm{~A}$ \\
\hline & 6-fold Scaler & Kinetics & 3610 \\
\hline & Output Register & Jorway & 41 \\
\hline & Interrupt Register & Ortec & IR026 \\
\hline & Clock & Jorway & 217 \\
\hline & $\begin{array}{l}\text { 12-fold Analog-ti- } \\
\text { Digital Converter }\end{array}$ & Lecroy & $2249 \mathrm{~A}$ \\
\hline
\end{tabular}


cables to and from each module. CAMAC modules are plugged inio a CAMAC "crate," which likewise provides a common power source for the modules. In addition, the crate provides common data paths ("data ways") tor reading and controlling such modules by means of a microprocessor. NIM modules are shown as wide rectangles; CAMAC as narrow ones.

The CAMAC modules were operated in a LeCroy 3500[20] data acquisition system that provides a small CAMAC crate. This system is very versatile and provides many options and features not pertinent i.o this experiment. It can accommodate up to eight cAMAC modules under control of its microprocessor (INTEL 8085). In addition, there is accessible internally an arithmetic processing unit (Advariced Micro Devices APU9511). Besides program memory, there are 16K 3-byte words of data memory.

We will now describe the data acquisition system with reference to Figure 5 .

The eight gamma channels were connected to eight of the ten discriminators available in the two Phillips Model 715 discriminators. The analog gamma signals (with attenuation and delay) were also connected to eight inputs of the LeCroy Model 2249A 12-channel integrating analog-to-digital converter (ADC). The eight gamma discriminato outputs were led to eight contiguous inputs (upper byte or lower byte) of the LeCroy Model 2341A 16-channel coincidence register.[20]

The fission counter was connected to one of the two remaining discriminators. That output goes to gate the coincidence register. Another output goes to trigger the one remaining discriminaur. The reason for this will be explained later. 
Signal delays in the system are adjusted simply by changing cable length. Only cable RGl74 was used for signal handling. This cable has a signal propagation velocity of approximately $20 \mathrm{~cm} /$ nanosecond. For comparison, the velocity of light is $30.0 \mathrm{~cm} /$ nanosecond in free space, and $19 \mathrm{~cm} /$ nanosecond in the plastic scintillator.

On the time scale of interest, there were measurable di:ferences in the eight detectors. The lengths of the cables from the gamma discriminators to the coincidence register were adjusted to bring gamma pulses into simultaneity within $\pm 1 / 10$ nanosecond (or, equivalently, \pm 2 cm of cable length). 


\section{DATA ACQUISITION}

With the LeCroy 3500 , data acquisition procedures can be programmed either in FORTRAN or in assembly language. In the interest of reducing deadtime, all of these experiments acquired data through assembly language subroutines. For convenience, the overall data acquisition was managed by a FORTRAN program that read operator input (for example, count time) from the keyboard, cleared data memory, and then called the subroutine for actual data acquisition. At the end of the specified count time, control was returned to the FORTRAN calling program. This then performed preliminary analysis as may have been programmed and wrote out the results on the printer. At program end, the data as well as some interim results remained protected in data memory for the use of a subsequent program. The data acquisition software is presented in detail in Appendix $C$.

We now describe data acquisition with respect to a single fission event. (Refer to Figure 5.) The data cycle starts from the quiescent condition when a fission occurs in the fission counter and causes a signal pulse greater than the threshold of the associated discriminator. The discriminator has multiple outputs, one of which goes to the interrupt register and signals the waiting program that an analyzable event has occurred. The acquisition program then, by means of the output register, turns on an inhibit signal that shuts off 
further input until the inhibit is revoked at the end of data processing. The output of the fission discriminator also triggers a gate that acts as another inhibit. The two inhibit signals are combined by a logical $O R$ (in the fan-in/fan-out module). The reason for the apparent duplication is that the hard wired INHIBIT (through the gate generator) can be applied much more quickly than the software inhibit; on the other hand, it is necessary to have software control of the INHIBIT during initialization and output.

The output of the fission discriminator (a logic pulse of approximately 8 ranoseconds duration) also goes through a delay line tu gate the coincidence register. The width and arrival time of the gate signal were adjusted to accept any pulse arriving within 3 nanoseconds after the peak.

All, some, or none of the eight gamma scintillation detectors may have detected a gamma at the instant of fission. After the gate has closed, we are left with a byte of data in the coincidence register. We will refer to this byte as a "bit pattern". This bit pattern contains a "l" corresponding to each channel that had a signal pulse in coincidence with the gate and a "0" for each of the other channels. For example, if only detectors 1,3 , and 4 had coincident gamma signals, the bit pattern would be 0000 1101. This has the numerical value of $13_{10}$ and the data acquisition program would accordingly increment by 1 data memory 13, indicating one more occurrence of an event having this bit pattern. It requires 256 memory locations (0-255) to accent all the possible values of the bit pattern. 
Earlier we alluded to a sc sond discriminator in the fission detector channel. Its purpose is to provide a separately adjustable gate to control the LeCroy 2249A ADC.[20] The gate is about 15 nanoseconds wide to permit proper integration of the analog pulses from the detectors.

At the instant the gates close, we have in the coincidence register a bit pattern as described above. Every "l" in that bit pattern indicates the occurrence of a gamma pulse that met:

1) the energy criterion of being greater in amplitude than the gamma discriminator threshold; and

2) the time criterion by arriving while the gate was open at the coincidence register.

We have in each of the 8 ADC channels in use a 10-bit number corresponding to the integrated amplitude of the signal that arrived while the ADC was gated on. Because the ADC gate is wide enough to permit integration of the photomultiplier pulses, there is the possibility that a neutron signal will be accepted. Even if there is no signal at all, there is an unavoidable "pedestal" due to the integrating characteristic of the ADC. The pedestals and neutron pulses have small effect, because the data acquisition software reads only those ADC channels corresponding to bona fide gamma events as identified by the above two criteria. The evaluation of these biases will be discussed later.

The program sums (in our hypothetical fission event) the ADC values in channels 1,3 , and 4, (these are 10-bit numbers) and divides by 3 to obtain the average amplitude for the three gammas recorded. 
This is also divided by 4 to scale the number down to one byte ( 8 bits) for economy of handing time and data memory. Suppose further, that the above steps result in the number 48 . This scaled average amplitude is stored in the data memory in a block reserved for multiplicity 3 . That is, data memory location 48 in block 3 is incremented by 1 .

This involved storage operation takes place after each event and the input is protected from additional signals as long as necessary by the inhibit control signal as described earlier. Conc urrently, the program counts the number of analysis cycles for later comparison with the scaler count of initiating signals. At the end of data storage, the necessary clear signals are generated and the INHIBIT is removed to wait for the next fission event.

At the end of the specified count time, control returns to the FORTRAN calling program. This then prints out preliminary results and allows the operator to (1) quit, (2) repeat, (3) change count time and repeat, or (4) output a statistical summary of the energy data. This summary includes for each block (multiplicity) a total count, maximum ordinate, average energy (first moment), and the sigma of the distribution. After the option 4 printout, the operator is offered the choice of the first three above options or calling by block number for plotting any of the eight spectra.

Figure 6 is an example of the output obtained from each data run. The options mentioned in the last paragraph appear only on the video display and not on the printout. We will explain the printed data in detail because there are a large number of internal checks to detect inconsistencies. Line 1 gives the number of cycles as 
determined by a running tally of the number of times the analysis program was initiated. Also in the first line is the counting time. In this case, the time is to be adjusted by a factor of 10 (it should be 21,627 ) because a ten times lower than normal clock rate was selected. For comparison with the number of analysis cycles, Line 2 gives the number of gates as counted by a scaler external to the analysis program.

Line 3 gives the number of gamma events counted by each of the eight detectors as determined from the stored bit patterns; (failure of a channel is obvious); and the sum of these eight numbers (total gamma events) is shown in Line 9. Line 4 is the probability of an event per cycle for each detector (Line 3 divided by the number of cycles). Line 5 gives the relative efficiencies of the eight detectors as estimated from the "miss" probabilities (to be explained later). Line 6 gives the product of all eight "miss" probabilities.

Line 7 gives the number of times each multiplicity ( 0 to 8 ) was observed. These nine numbers were obtained from the bit patterns; the sum of Line 7 entries is given in Line 11 as a third check on the number of cycles (against Lines 1 and 2). Also, the entries in Line 7 are each multiplied by their respective multiplicities and summed to obtain the total number of gammas (Line 10) for comparison with Line 3. Line 8 gives the respective probabilities of the multiplicities (0 to 8 ) obtained by dividing Line 7 by Line 2. These are the results carried over to the program that infers the emission multiplicity.

Line 12 is a meaningless combination of all those cycles in which at least one gamma was detected. However, it will be seen that 
the first data entry in Line 12 plus the first entry in Line 7 equals Line 2 as it should number of cycles with gammas plus the number of cycles without gammas equals the total number of cycles). The entries in the COUNTS column (in Lines 13 through 20) correspond respectively to the last eight entries in Line 7 . As mentioned above, Line 7 was obtained from the stored bit pattern; the data under COUNTS was obtained by summing in each of the spectral blocks.

"CMAX" is the maximum ordinate in each of the spectral blocks; the prugram uses this number to establish the vertical scale on output plots. "EBAR" is the first moment (average energy) of a given block in arbitrary units. "SIGMA" is the root-mean-square deviation about "EBAR". Repeating in other words what was described earlier, each datum stored in spectral block $M$ is actually the average energy of $M$ different signals coming from $M$ different detectors. Thus, in block $M$, the dispersion about the mean should go about as $1 / \sqrt{M}$. Looking at the last column we see that $\sigma$ for $M=4$ is about half that for $M=1$, and that $\sigma$ for $M=8$ is about half that for $M=2$. The difference between blocks is illustrated in Figure 7 . 


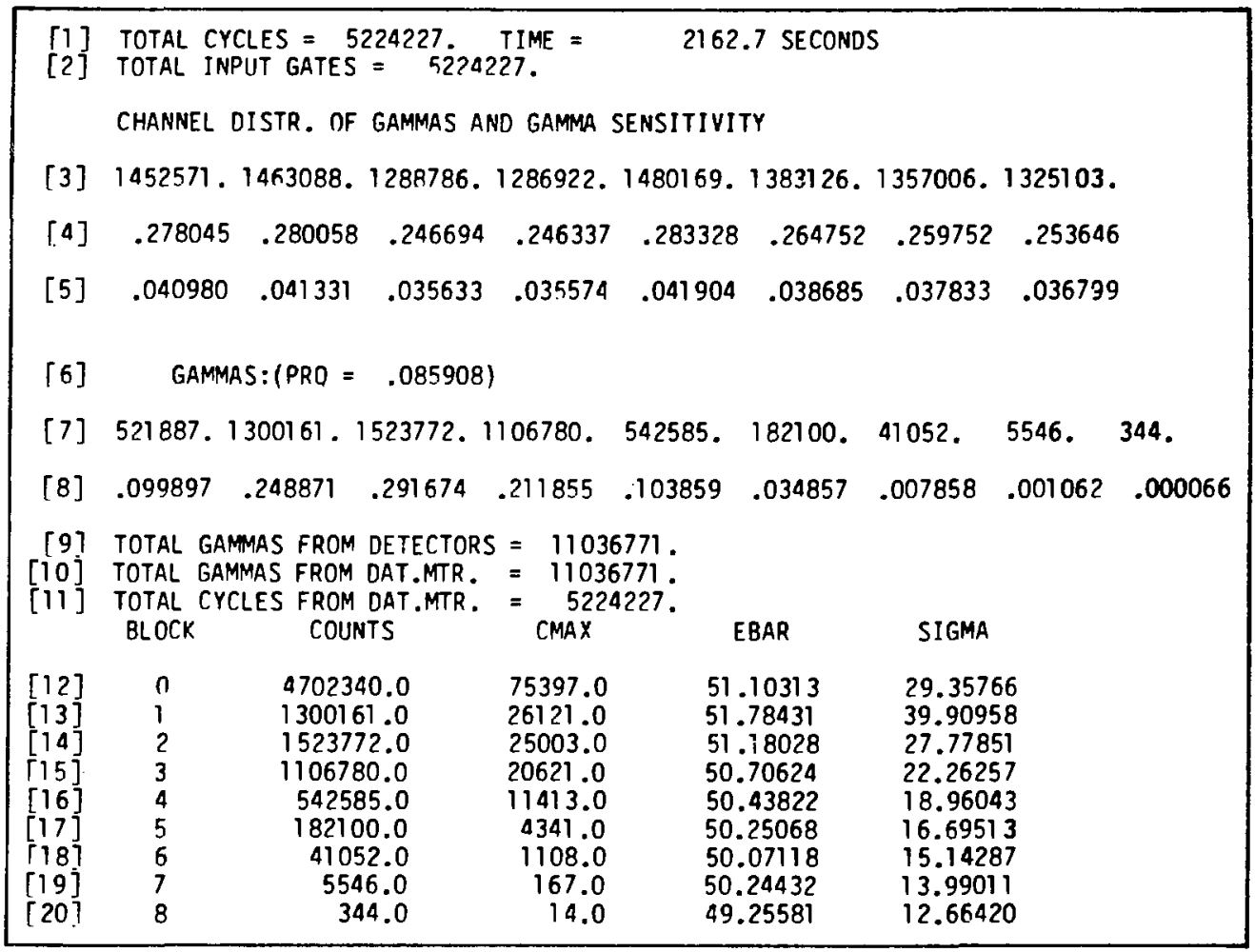

Fig. 6. Typical output from data acquisition program.

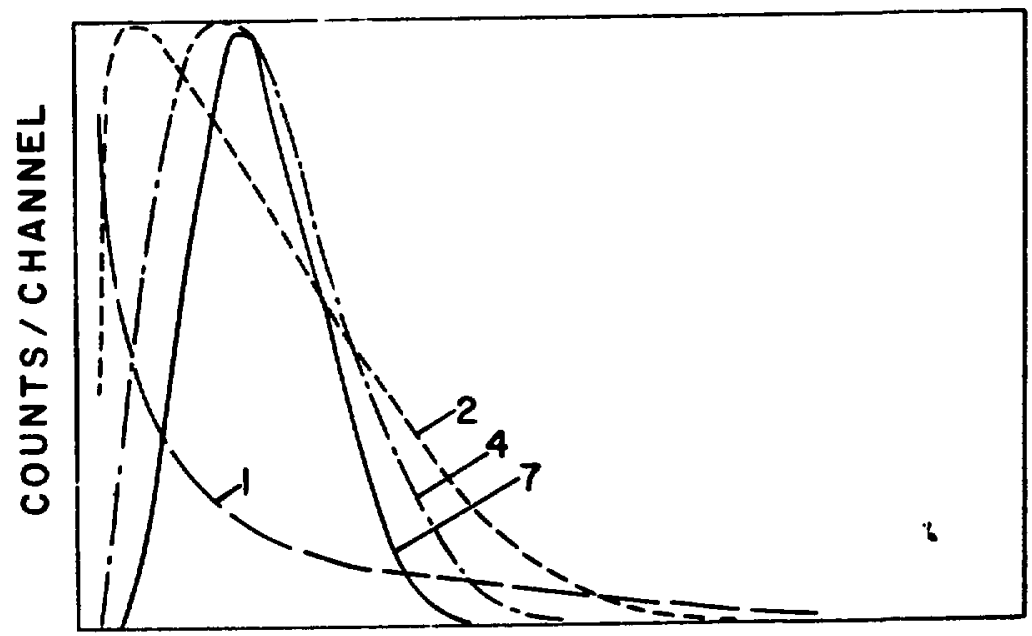

ENERGY (arb. scale)

Fig. 7. Comparison of stored spectra for various multiplicities. 
OPERATIONAL CONSIDERATIONS

\section{General}

We wish to detect "fission" gamas with a high efficiency. Some consideration needs to be given to what is meant by "fission" gammas. The idea of absolute simultameity falters when we consider the fission phenomenon on the time scale of interest here. For example, Rumpold et al.[8] have been able to classify several specific fission gammas as to whether they are emitted less than 1 picosecond after fission, between 1 picosecond and 2 nanoseconds, or after 2 nanoseconds. Bowman et al.[3] have done a similar experiment and most of the lines they studied, in general not the same as those of Rumpold[4] are assigned half lives less than $1 / 2$ nanosecond. In general, our results accord qualitatively with those of Bowman. It appears that for the purposes of our experiment, effectively all of the fission gammas appear in less than half a nanosecond. This is based on the observation that our time resolution curves taken between the fission counter and one of the gamma detectors was not substantially wider than the time resolution between two of the gamma detectors when the source was ${ }^{60}$ Co. This source emits two gamma rays that are reported to be connected by a half life of approximately 1 picosecond. 


\section{Energy Calibration}

Establishing an energy scale for the discriminators on the eight main counting channels is complicated by the poor energy resolution of plastic scintillators. As Nardi[21] points out, the principle gamma interaction with these materials is by compton scattering. For a monoenergetic source such as ${ }^{137} \mathrm{Cs}$, the resulting spectrum is a poorly resolved peak corresponding to the energy of the Compton electrons. The indistinct "Compton edge" on the right hand snoulder of this peak corresponds to the maximum energy transfer to an electron in the scintillator. For the $662 \mathrm{keV}$ gamma from ${ }^{137} \mathrm{Cs}$, this maximum energy is about $480 \mathrm{keV}$. The ${ }^{137} \mathrm{Cs}$ line was used to define the energy scale in the main scintillator channels. Because Compton scattering[22] occurs with a range of energy transfers from the maximum down to zero, it is possible for any given discriminator setting to miss even high energy gammas. The relationship between the shoulder (Compton edge) of the spectrum is not Iinear with the peak gamma energy.

The primary emphasis in this experiment is on high efficiency in the gamma detectors. For this reason, as well as the general characteristics of plastic scintillators, we have depended on others' results for values of $\bar{n}$, the average number of gammas emitted in fission of ${ }^{252} \mathrm{Cf}$. These are the last two entries in Table 1.

Energy

Threshold

(keV)

140

85
$7.80 \pm 0.3$

$8.32 \pm 0.4$ $\bar{n}$

Source

Verbinski [5]

Pleasanton[6]
Corresponding

compton edge (kev) 
The measurements reported here were all made at a gamma threshold corresponding to one or the other of the two above Compton edge energies.

\section{Timing}

The LeCroy Model 2341 A coincidence register provides 16 inputs. We used only eight at a time, either the top or bottom half. The gate must precede data pulses by at least 3 nanoseconds. The exact opening time of the gate is not critical but the closing time is. on the time scale of interest here, the transition time of the discriminator output (approximately $I$ nanosecond) and response time (turn-off) of the coincidence register are quite significant when we undertake to set the gate so that the effective "turn-off time" is fixed to about $1 / 2$ nanosecond.

All set-up operations involving timing required the use of auxiliary equipment not used in the experiment proper. This consisted of a time to pulse height converter (TPC) that was used in conjunction with the normal pulse height analysis function of the Lecroy system. The fission counter signal (after discrimination) started the time interval that was terminated by the discriminator signal from one of the gamma channels. The result of this analysis is shown in Figure 4. The peak on the left represents the distribution of gamma events in time while the hump on the right similarly represents the fast neutron events. The time scale is approxmately 48 picoseconds per channel. By adjusting cable lengths, we brought all eignt channels into simultaneity within approximately two channels. (One centimeter of cable corresponds to about one channel.) 
Without changing the zero time reference in the TPC, we then brought the gate signal from another output of the fission counter discriminator to the "stop" input of the TPC. By adjusting this cable length, we were able to set the leading edge of the gate at a point some 6 nanoseconds ahead of the gamma peak. The exact position is not critical.

What is critical is the end of the gate because it must be positioned to exclude fast neutrons while losing as few gammas as possiole. This sensitive timing depends on the interaction between the fall time of the gate signal and the turn-off characteristic of the coincidence register.

The first step was to bring a copy of the fission counter discriminator pulse from a separate simultaneous output to the "stop" input of the TPC (always mainudining the same zero time reference). By adjusting the cable length, the leadine edge of this "decoy" signal was placed exactly at the point on the time scale at which we wanted to close the gate against neutron pulses. With all cable lengtins fixed, this decoy signal was led to the coincidence register in place of one or another of the gamma input signals. The regular data acquisition program was run. In the channel with the substitute signal, it was immediately obvious if the gate was missing all the decoy pulses (gate too short) or counting a decoy pulse at every cycle (gate too long.) By trial and error, the duration of the (discriminator output) signal was adjusted to count about half of the decoy pulses. This corresponded to having the trailing edge of the gate at the chosen cut-off position. This method gives a proper cut-off time 
taking into account the real, combined characteristics of the various components.

\section{Detector Efficiency}

It would be highly misleading to attempi to measure counter efficiency by the usual technique of counting gammas first with the source present and then with it absent. We need the detection efficiency for prompt fission gammas. The straightforward technique of subtracting gross background from gross count would include gammas from two other origins extraneous to our purpose. First, it would include decay gammas from long-lived fission products accumulating since the fabrication of the ${ }^{252} \mathrm{Cf}$ source. Second, it would include the numerous gammas associated with alpha decay of ${ }^{252} \mathrm{Cf}$. (This is. very substantial source because there are about 30 times as many alpha decays as spontaneous fissions.)

It might be supposed that the gamma counter could be gated by fissions and that could lead to a reasonable measurement of the counter efficiency. One would simply divide the coincident count rate by the trigger rate getting the probability of a count for each fission, then divide that by $\bar{n}$ to get the efficiency. However, this raises another problem. At the efficiencies involved in this experinent (approximately 0.035), it is easily possible for two or more gammas from the same fission to strike the same detector resulting in a lower estimate of the efficiency than the true value.

There is, however, a relatively clean way to estimate the efficiency of the detectors under the actual conditions of the experiment. 
We accept from the literature that ${ }^{252}$ Cf emits 7.80 [5] or $8.32[6]$ gammas per fission, depending on the threshold, and we assume for this purpose that this is the average of a Poisson distribution.

When a fission occurs and emits $n$ gammas, the probability of not registering a count is the probability that all gammas miss the detector (or traverse it without registering.) This miss probability is:

$$
P_{\text {miss }}=(1-\varepsilon)^{n}
$$

where $\varepsilon$ is the efficiency for detecting a single fission gammat the current discriminator setting. Summing up the $P_{\text {miss }}$ for all values of $n$ under the Poisson distribution we have:

$$
\begin{aligned}
& P_{\text {miss }}=\sum_{n=0}^{\infty} \frac{(\bar{n})^{n} e^{-\bar{n}}(1-\varepsilon)^{n}}{n !}=e^{-\bar{n}} \sum_{n=0}^{\infty} \frac{[\bar{n}(l-\varepsilon)]^{n}}{n !}=e^{-\bar{n}}\left(e^{\bar{n}} e^{-\bar{n} \varepsilon}\right)=e^{-\bar{n} \varepsilon} \\
& \text { so } \ln \left(P_{\text {miss }}\right)=-\bar{n} \varepsilon \\
& \text { and } \varepsilon=\frac{-\ln \left(P_{\text {miss }}\right)}{\bar{n}}
\end{aligned}
$$

The choice of $\bar{n}$ is not critical because these results have been used only to obtain the relative efficiencies of the eigint gama detectors. 
INTERPRETATION OF DATA

The interpretation of the multiplicity data is "based on the following equation derived by Van der Werf[23]. Refer to Appendix $B$. Some symbrls have been substituted for convenience. Assuming an array of $\mathrm{N}$ identical detectors of average efficiency $\varepsilon$ counts a shower of $G$ gammas, we have the following probability of triggering exactly i detectors (that is, the emission multiplicity is $G$ and the detection multiplicity is $M$ ):*

$$
P_{N}(G, M)=\left(\begin{array}{l}
N \\
M
\end{array}\right) \sum_{\ell=0}^{M}(-1)^{M-\ell}\left(\begin{array}{c}
M \\
\ell
\end{array}\right)[1-(N-\ell) E]^{G} .
$$

* For historic reasons, we have to this point used the symbol $\bar{n}$ to represent the average number of gammas emitted in fission. Henceforth, for reasons of clarity and mnemonic convenience, we will use the following symbols:

$\bar{G} \quad$ average number of gammas emitted

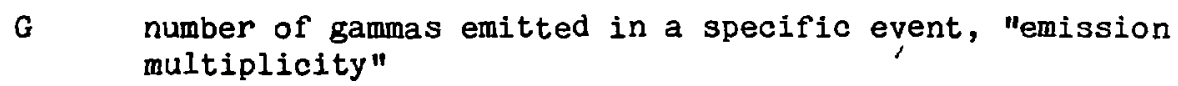

$\pi(G)$ probability that exactly $G$ gammas are emitted

$N$ number of gamma detectors in the array

M number of detectors triggered in a given event, "detection multiplicity"

$P(M)$ probability that exactly $M$ detectors are triggered

$M$ number of gammas actually striking the $M$ triggered :

detectors, "impact multiplicity" 
A careful distinction must be made between $M$ the number of detectors triggered and $M^{\prime}$ the actual number of gammas striking those $M$ detectors; (obviously $G \geq M^{\prime} \geq M$ ).

We assume a hypothetical exission distribution (a model) and some starting values for the parameters of the distribution $\left(C_{1}\right.$, $\left.C_{2}, \ldots\right)$

$$
\pi(G)=\pi\left(G, C_{1}, C_{2}, \ldots\right)
$$

and calculate the expected probability of triggering exactly $M$ detectors

$$
P_{N}(M)=\sum_{G=M}^{\infty} \pi(G) P_{N}(G, M)
$$

Written explicitly:

$$
P_{N}(M)=\sum_{G=M}^{\infty} \pi(G)\left(\begin{array}{l}
N \\
M
\end{array}\right) \sum_{\ell=0}^{M}(-1)^{M-\ell}\left(\begin{array}{c}
M \\
l
\end{array}\right)[\ell-(N-\ell) \varepsilon]^{G} .
$$

We have from the experiment a set of observed probabiljties for the distribution of $M$ corrected for false counts caused by scattering.

$$
P_{N}^{O}(M) \quad, \quad(0 \subseteq M \Xi N) \quad
$$

$\bar{G}(M)$ average emission multiplicity for a $g^{i v e n} M$

$\bar{M}^{\prime}(M)$ average impact multiplicity for a given $M$

$\bar{E}(M)$ average gamma energy per triggered detector for a given $M$

$\bar{E}^{\prime}(M)$ average energy per gamma for a given $M$

c effective average detector efficiency for fission gammas 
The numerical procedure is shown in the flow chart, Figure 8 . The program adjusts the parameters of the chosen model to obtain 3 "best fit" by the criterion of minimum weighted least squares. The minimization of the weighted squares is performed by a comprehensive routine written by J. P. Chandler of Indiana University in 1966. The brevity of the flowchart may be misleading. The data analysis program includes many options and the FORTRAN listing requires some 20 pages single spaced. For that reason, it has not been included.

The output from this computation is extensive and includes the experimental data $P_{N}^{O}(M)$, the "best fit" results from the model $P(M)$, the absolute difference between the two, and the relative deviation, as well as the converged value of the summed square deviations.

After the problem has converged, the program computes the matrix of the two dimensional probabilities:

$$
P_{N}(G, M),
$$

the probability that in a given fission exactly $G$ gammas are emitted and exactly $M$ detectors are triggered. Finally, the program calculates an answer to the Bayesian question:

If $M$ detectors were triggered, what was the average number of gammas emitted in the fission event? The calculation is:

$$
\bar{G}(M)=\frac{\sum_{G=M}^{\infty} G \cdot P(G, M)}{\sum_{G=M}^{\infty} P(G, M)}
$$




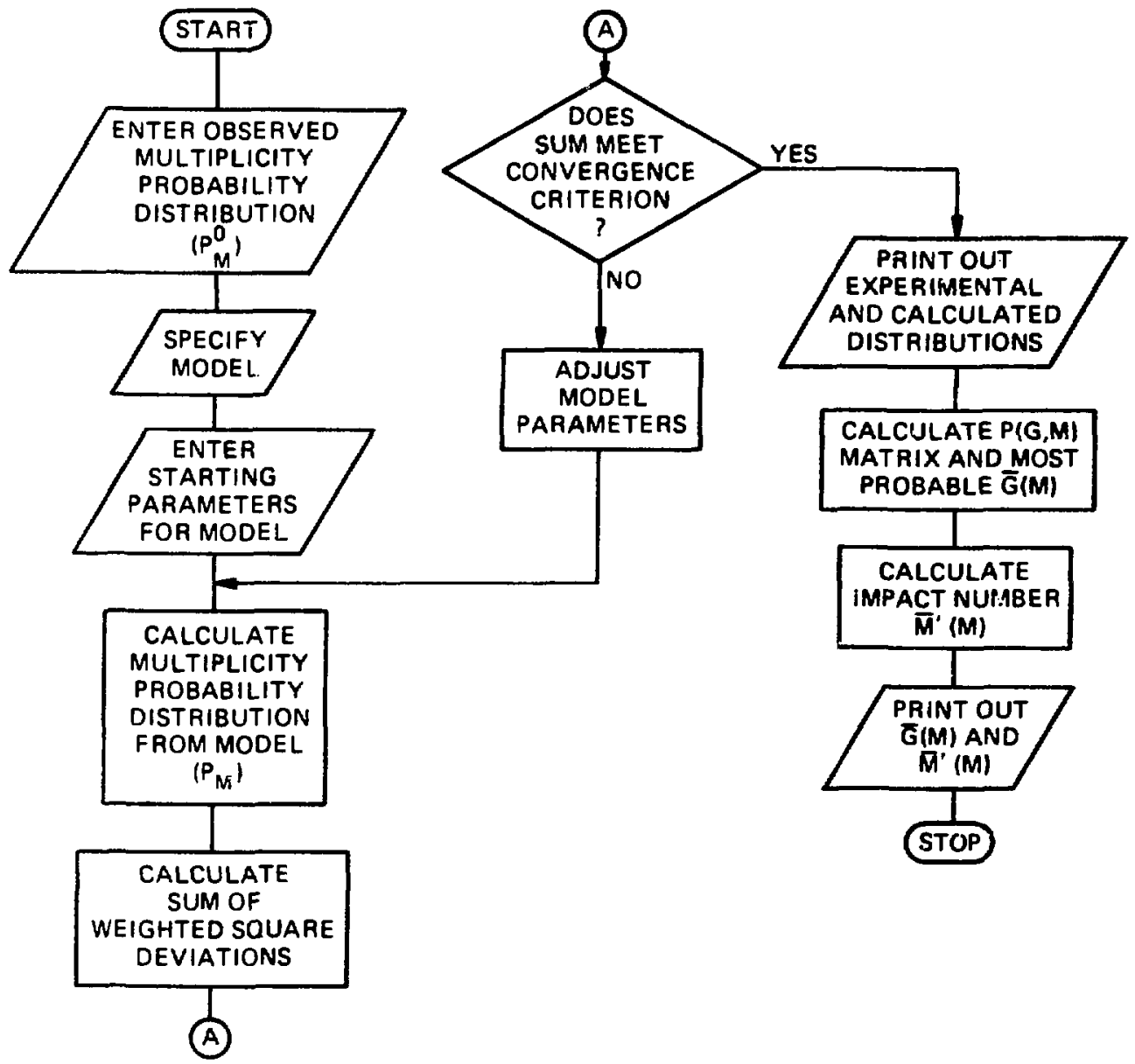

Fig. 8. Flow chart for the data analysis program. 
The program also computes the average impact multiplicity, $\bar{M} \cdot(M)$, as a function of detection multiplicity. As shown in Equation 38 in Appendix B, we have

$$
P_{N}\left(G, M^{\prime}, M\right)=\left(\begin{array}{c}
N \\
M
\end{array}\right)\left(\begin{array}{c}
G \\
M^{\prime}
\end{array}\right)(1-N E)^{G-M^{\prime}} \sum_{\ell=0}^{M}(-1)^{M-l}\left(\begin{array}{c}
M \\
l
\end{array}\right)(l \in)^{M^{\prime}},
$$

which gives the probability that out of a shower of $G$ gammas on $N$ detectors, exactly $M^{\prime}$ of the gammas trigger $M$ detectors. Using the inferred emission distribution $\pi(G)$ we can calculate $\bar{M} '(M)$.

$$
\bar{M}^{\prime}(M)=\frac{\sum_{M^{\prime}=M}^{\infty} \sum_{G=M}^{\infty} M^{\prime} \cdot \pi(G) \cdot P_{N^{\prime}}\left(G, M^{\prime}, M\right)}{\sum_{M^{\prime}=M}^{\infty} \sum_{G=M}^{\infty} \pi(G) \cdot P_{N}\left(G, M^{\prime}, M\right)} .
$$

The purpose is to achieve a better correlation of energy with multiplicity. As previously described, the data acquisition program yields $\bar{E}(M)$, the total gamma energy of the $M$-fold event divided by $M$. However, on the average, this total energy is the response to $\bar{M}$ ' gammas that takes into account the fact that detectors frequently experience multiple gamma impacts from the same fission. Hence $\bar{E}$ from the data acquisition program must be corrected to

$$
E^{\prime}(M)=\bar{E}(M) \frac{M}{\bar{M} \cdot(M)}
$$


RESULTS

In Table 2 we present six sets of data enumerated below:

\begin{tabular}{|c|c|c|c|}
\hline $\begin{array}{l}\text { Run } \\
\text { Number }\end{array}$ & $\begin{array}{c}\text { Gamma Discriminator } \\
\text { Threshold (keV) }\end{array}$ & $\begin{array}{l}\text { Average No. } \\
\text { of Gammas }\end{array}$ & $\begin{array}{c}\text { Fission Discriminator } \\
\text { Setting }\end{array}$ \\
\hline 52 & 140 & 7.80 & Optimum \\
\hline 53 & 140 & 7.80 & Optimum \\
\hline$\$ 4$ & 140 & $7.80 ?$ & Biased \\
\hline 36 & 85 & 8.32 & Optimum \\
\hline S7 & 85 & 8.32 & Optimum \\
\hline S8 & 85 & $8.32 ?$ & Biased \\
\hline
\end{tabular}

In Table 2 there are typically four entries for each run under each multiplicity:

l. the number of times that multiplicity was observed (run data);

2. the estimated number of events that are "demoted" to the next lower multiplicity;

\footnotetext{
* "Optimum" refers to a setiing between the humps of the fission fragment energy spectrum that is considered to be the best availale for triggering on a representative distribution of fission events. "Biased" means a discriminator setting that accepts approximately the upper 10\% of all pulses in the nigh energy hump of the fission fragment spectrum.
} 
TABLE 2

RAW DATA SHOWN WITH CORRECTION FOR FALSE COUNTS AND RESULTING CORRECTED DATA

\begin{tabular}{|c|c|c|c|c|c|c|c|c|c|c|}
\hline & $\begin{array}{l}\text { Total } \\
\text { Events }\end{array}$ & 0 & 1 & 2 & $\begin{array}{c}\text { ction Mu } \\
3\end{array}$ & $\begin{array}{l}\text { licity } \\
4\end{array}$ & 5 & 6 & 7 & 8 \\
\hline $\begin{array}{l}\frac{\text { Run } 52 \text { at } 140 \mathrm{keV}}{\text { raw data }} \\
\text { correction to } M-1 \\
\text { correction from } M+1 \\
\text { corrected data }\end{array}$ & $9,415,495$ & $\begin{array}{l}1,173,314 \\
1,173,314\end{array}$ & $\begin{array}{r}2,647,957 \\
54,867 \\
2,702,824\end{array}$ & $\begin{array}{r}2,782,169 \\
-54,867 \\
98,332 \\
2,825,634\end{array}$ & $\begin{array}{r}1,781,587 \\
-98,332 \\
76,552 \\
1,759,807\end{array}$ & $\begin{array}{r}761,227 \\
-76,552 \\
33,314 \\
717,989\end{array}$ & $\begin{array}{r}221,502 \\
-33,314 \\
8,558 \\
196,746\end{array}$ & $\begin{array}{r}42,617 \\
-8,558 \\
1,228 \\
35,287\end{array}$ & $\begin{array}{r}4,873 \\
-1,228 \\
76 \\
3,721\end{array}$ & $\begin{array}{l}248 \\
-76 \\
172\end{array}$ \\
\hline $\begin{array}{l}\frac{\text { Run } 53 \text { at } 140 \mathrm{keV}}{\text { raw data }} \\
\text { correction to } M-1 \\
\text { correction from } M+1 \\
\text { corrected data }\end{array}$ & $12,314,425$ & $\begin{array}{l}1,478,350 \\
1,478,350\end{array}$ & $\begin{array}{r}3,417,192 \\
70,806 \\
3,487,998\end{array}$ & $\begin{array}{r}3,651,645 \\
-70,806 \\
129,106 \\
3,709,945\end{array}$ & $\begin{array}{r}2,373,541 \\
-129,106 \\
102,073 \\
2,346,508\end{array}$ & $\begin{array}{r}1,027,015 \\
-102,073 \\
45,005 \\
969,947\end{array}$ & $\begin{array}{r}301,176 \\
-45,005 \\
11,650 \\
267,821\end{array}$ & $\begin{array}{r}58,349 \\
11,650 \\
1,684 \\
48,383\end{array}$ & $\begin{array}{r}6,775 \\
-1,684 \\
105 \\
5,196\end{array}$ & $\begin{array}{r}382 \\
-105 \\
277\end{array}$ \\
\hline $\begin{array}{l}\frac{\text { Run S4 at } 140 \mathrm{keV}}{\text { raw date }} \\
\text { correction to } M-1 \\
\text { correction from } M+1 \\
\text { corrected data }\end{array}$ & $3,117,564$ & $\begin{array}{l}429,021 \\
429,021\end{array}$ & $\begin{array}{r}919,589 \\
19,054 \\
938,643\end{array}$ & $\begin{array}{r}912,595 \\
-19,216 \\
32,216 \\
925,757\end{array}$ & $\begin{array}{r}554,510 \\
-32,216 \\
23,753 \\
546,047\end{array}$ & $\begin{array}{r}225,474 \\
-23,753 \\
9,815 \\
211,536\end{array}$ & $\begin{array}{r}62,980 \\
-9,815 \\
2,418 \\
55,583\end{array}$ & $\begin{array}{r}11,981 \\
-2,418 \\
345 \\
9,908\end{array}$ & $\begin{array}{r}1,344 \\
-345 \\
21 \\
1,020\end{array}$ & $\begin{array}{r}70 \\
-21 \\
59\end{array}$ \\
\hline $\begin{array}{l}\frac{\text { Run } 56 \text { at } 140 \mathrm{keV}}{\text { rew data }} \\
\text { correction to } \mathrm{M}-1 \\
\text { correction from M+1 } \\
\text { corrected data }\end{array}$ & $16,341,425$ & $\begin{array}{l}1,628,434 \\
1,628,434\end{array}$ & $\begin{array}{r}4,060,708 \\
135,105 \\
4,195,813\end{array}$ & $\begin{array}{r}4,772,499 \\
-135,105 \\
270,940 \\
4,908,334\end{array}$ & $\begin{array}{r}3,465,441 \\
-270,940 \\
236,761 \\
3,431,262\end{array}$ & $\begin{array}{r}1,695,870 \\
-236,761 \\
115,917 \\
1,575,026\end{array}$ & $\begin{array}{r}572,080 \\
115,917 \\
33,805 \\
489,968\end{array}$ & $\begin{array}{r}127,924 \\
-33,805 \\
5,501 \\
99,620\end{array}$ & $\begin{array}{r}17,384 \\
-5,501 \\
396 \\
12,279\end{array}$ & $\begin{array}{r}1,089 \\
-396 \\
693\end{array}$ \\
\hline $\begin{array}{l}\text { Run } 57 \text { at } 140 \mathrm{keV} \\
\text { raw data } \\
\text { correction to } M-1 \\
\text { correction from M+1 } \\
\text { corrected data }\end{array}$ & $5,224,227$ & $\begin{array}{l}521,887 \\
521,887\end{array}$ & $\begin{array}{r}1,300,161 \\
43,258 \\
1,343,419\end{array}$ & $\begin{array}{r}1,523,772 \\
-48,258 \\
86,499 \\
1,567,013\end{array}$ & $\begin{array}{r}1,106,780 \\
-86,499 \\
75,618 \\
1,095,899\end{array}$ & $\begin{array}{r}542,585 \\
-75,618 \\
37,098 \\
504,065\end{array}$ & $\begin{array}{r}182,100 \\
-37,098 \\
10,746 \\
155,708\end{array}$ & $\begin{array}{r}41,052 \\
-10,746 \\
1,771 \\
32,077\end{array}$ & $\begin{array}{r}5,546 \\
-1,771 \\
126 \\
3,901\end{array}$ & $\begin{array}{r}344 \\
-126 \\
218\end{array}$ \\
\hline $\begin{array}{l}\text { Run S8 at } 140 \text { keV } \\
\begin{array}{l}\text { rarrection to } M-1 \\
\text { correction from } M+1 \\
\text { correctiod data }\end{array}\end{array}$ & $2,769,793$ & 294,488 & $\begin{array}{r}713,202 \\
23,729 \\
736,931\end{array}$ & $\begin{array}{r}809,830 \\
-23,729 \\
45,928 \\
832,029\end{array}$ & $\begin{array}{r}568,964 \\
-45,928 \\
38,765 \\
561,801\end{array}$ & $\begin{array}{r}271,289 \\
-38,765 \\
18,472 \\
250,996\end{array}$ & $\begin{array}{r}89,365 \\
-18,472 \\
5,254 \\
76,147\end{array}$ & $\begin{array}{r}19,818 \\
-5,254 \\
851 \\
15,415\end{array}$ & $\begin{array}{r}2,6^{\prime} 0 \\
-851 \\
60 \\
1,869\end{array}$ & $\begin{array}{l}177 \\
-60 \\
117\end{array}$ \\
\hline
\end{tabular}


3. the estimated number of exints that are "retrieved" from the next higner multiplicity; and

4. the corrected number of times the multiplicity occurred.

Items 2 and 3 above are corrections for gammas that, througr a Compton scattering, happen to trigger two detectors, thereby promoting an $M-f o l d$ event to $M+1$. The details of this correction are given in Section VII. The last item is entered for each multiplicity in the interpretative computation described earlier.

\section{Multiplicity}

We have summarized in Appendix $A$ the many different models tested against the experimental data. The criterion for fitting was the weighted least square deviation between the detection multiplicity data recorded and the detectior multiplicity data calculated from a given emission model. of the many models tried, the following "double Poisson" was the best:

$\pi(G)=C_{2} \frac{\left(C_{3}\right)^{G} e^{-C_{3}}}{G !}+\left(1-C_{2}\right) \frac{\left(C_{4}\right)^{G} e^{-C_{4}}}{G !}$

Next best was the skewed pseudo-Gaussian:

$$
\begin{aligned}
& \pi(G)=C_{1}(G)^{C_{2}} e^{-C_{3}\left(G-C_{4}\right)^{2}} \quad G \geq 1 \\
& \pi(0)=C_{5} e^{-C_{3}\left(-C_{4}\right)}
\end{aligned}
$$


All other models were vastly inferior. They resulted in values for $x^{2}$ two and three orders of magnitude greater than did the two above models.

The double Poisson was used to obtain all the subsequent reported results except in one case specifically noted. Figure 9 is an example of the output of the program CGAOl that was used to infer the emission gamma distribution. Referring to Figure 9, we see "FINAL VALUES OF $X(1)$. . ." These are respectively, the efficiency, C2, C3, and C4.

The block marked (I) contains the $\pi(G)$. The columns in block (2) are, respectively:

a. ("FOLD") - detection multiplicity.

b. ("EXP") - experimental probability (with previously described correction) of observing that multiplicity.

c. ("MODEL") - calculated probability (from model) of observing that multiplicity.

d. ("DEV") - difference between preceding two columns.

e. ("RDEV") - relative difference.

f. ("CHI") - Jifference between model and experiment expressed in sigmas (RMS deviations) on the original experimental data.

"CHISQ" is absolute $X^{2}$; "BIG CHI" is $X^{2}$ normalized to the number of events in the run. This is the value to be used for determini:goodness of fit. The final two lines of numerical output are, respectively, the average emission number $(\bar{G}(M), M=1$ to 8 , reading left to right) and the average inpact number $\left(\bar{M}^{\prime}(M), M=1\right.$ to 8$)$.

The results with the $140 \mathrm{keV}$ discriminator setting are given in Figure 10. It was not possible on this vertical scale to distinguish between the experimental results and the calculated results 


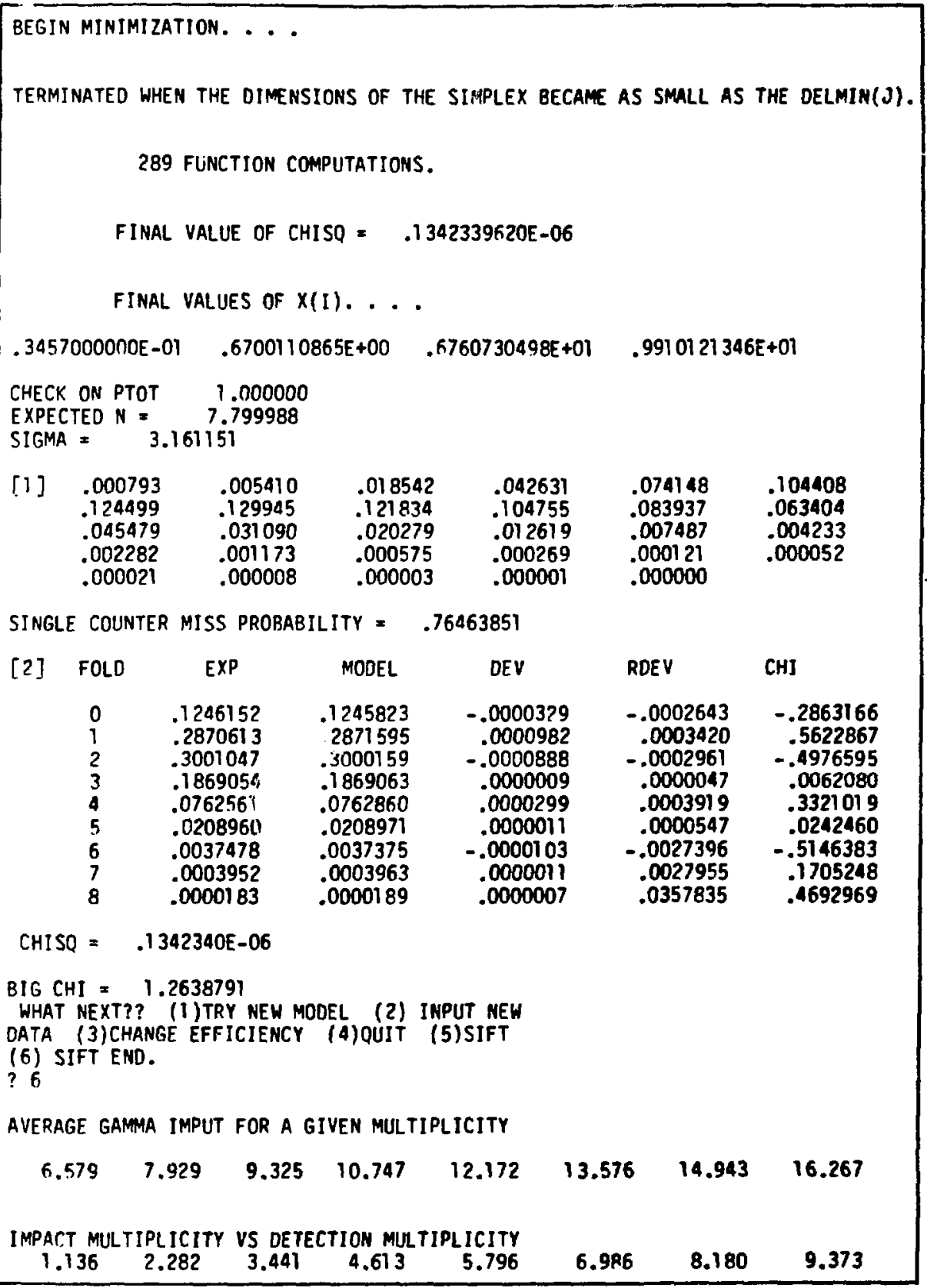

Fig. 9. Typical computer output from the data analysis program. 


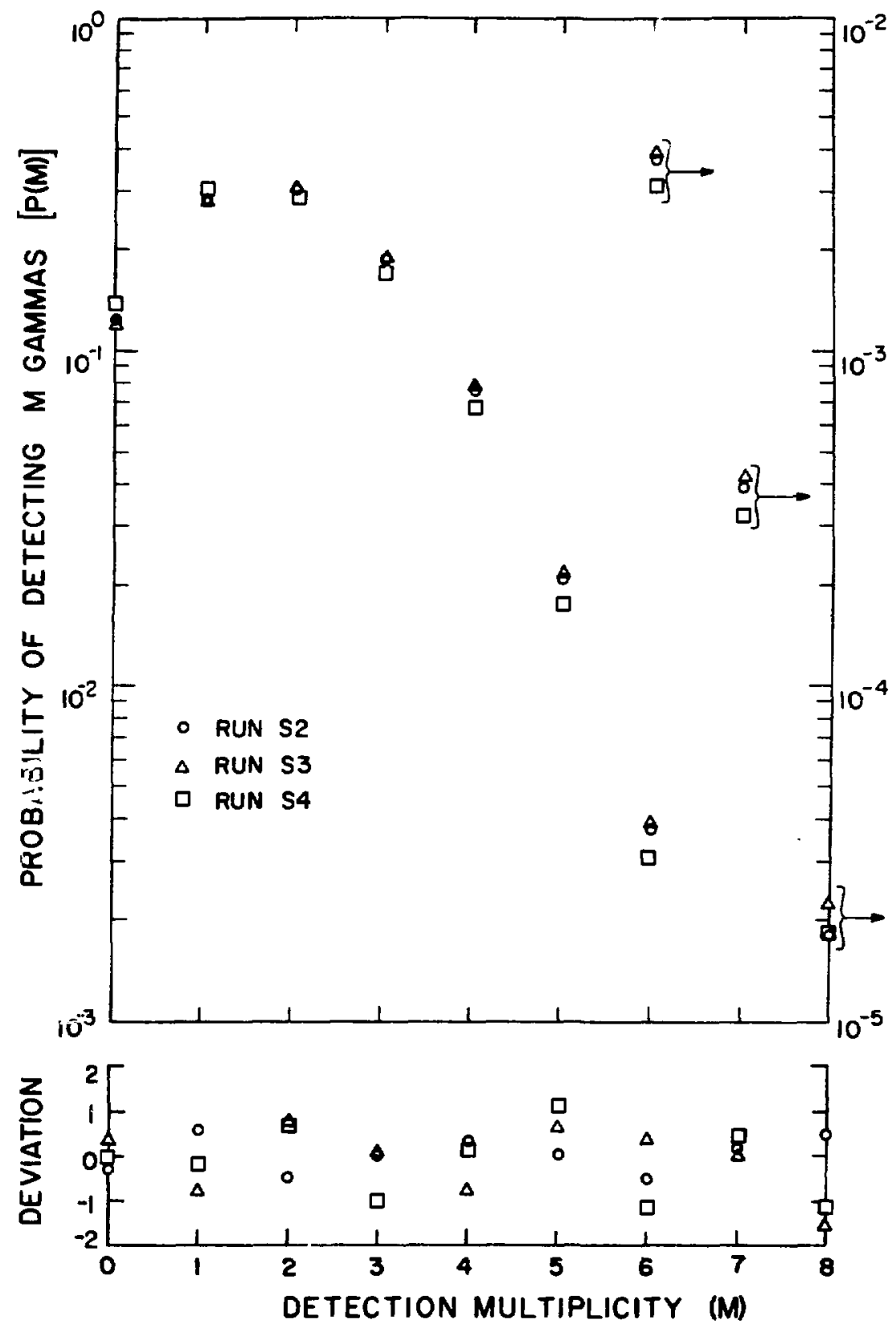

Fig. 10. Experimental results for measurements at $140 \mathrm{keV}$ threshold. The points in the upper graph represent both experiment and model since they differ by too small an amount to show on this scale. The lower graph shows the difference between experiment and model in units of standard deviation. 
obtained with the model. Instead, the corresponding deviations (ModelExperiment) are plotted in units of standard deviation in tne lower graph.

Figure 11 is the corresponding graph for the data obtained witn the $85 \mathrm{keV}$ threshold. The runs with the fission detector at a higher threshold, 54 (Figure 10) and S8 (Figure 11), snow relatively lower probabilities at higher multiplicities. This can bis attributed to fewer gammas or lower efficiency. Table 3 summarizes the results of fitting the model to the six sets of data.

We start with the nine probabilities for detection nultiplicities 0-8 inclusive. This represents only eight degrees of freedom because the probabilities are normalized to unity. Because three parameters are used in the model, the resulting values for $\chi^{2}$ are considered on the basis of 5 degrees of freedom. From a standard statistical table, we see that $\chi^{2}$ for 5 degrees of freedom should exceed 6.63 only $25 \%$ of the time. In Table $3, x^{2}$ exceeds 6.63 in only one of the six cases (S6). For $56, \chi^{2}$ is 7.49, which by interpolation snould be exceeded only $20 \%$ of the time; this still indicates a very reasonable fit. Hence, it is unlikely that we can improve the results obtained from this data by choosing a better model. However, it is entirely possible that another experiment using more detectors could improve the model because the greater number of detectors would afford more data points, but not proportionately so.

Figure 12 shows the emission distribution inferred from data sets S2, S3, and S4 (at $140 \mathrm{keV}$ threshold.) Figure 13 gives the same results for $S 6, S 7$, and $S 8$ ( $85 \mathrm{keV}$ threshold). 


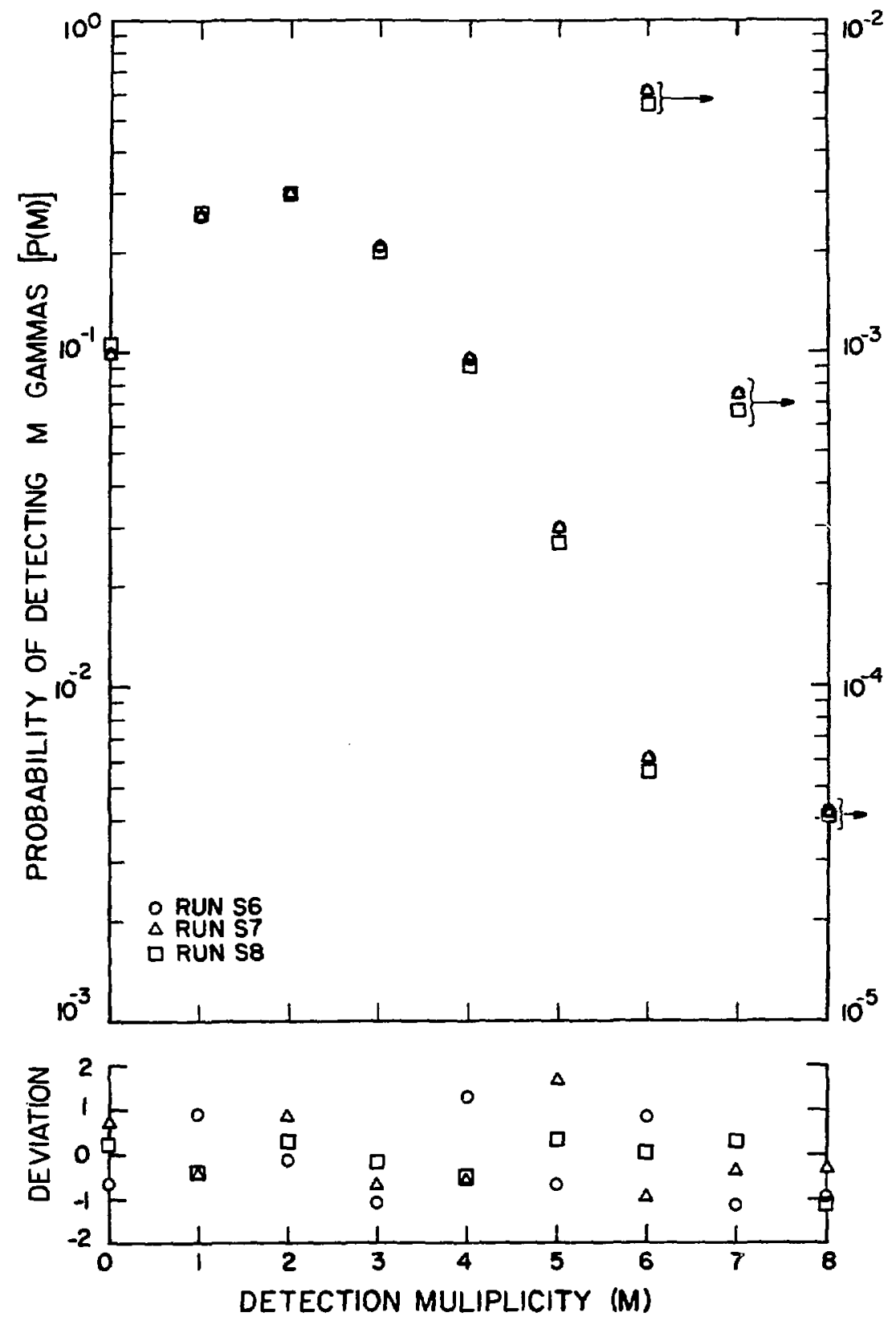

Fig. 11. Experimental results for measurements at $85 \mathrm{key}$ threshold. The points in the upper graph represent both experiment and model since they differ by too small an amount to show on this scale. The lower graph shows the difference between experiment and model in units of standard deviation. 
TABLE 3

RESULTS OBTAINED BY FITTING THE DOUBLE POISSON MODELa TO CORRECTED DATA (from Table 2)

\begin{tabular}{ccccccccc}
\hline $\begin{array}{c}\text { Gun } \\
\text { Number } \\
\text { Threshald } \\
(\mathrm{keV})\end{array}$ & $\varepsilon$ & $c_{2}$ & $c_{3}$ & $c_{4}$ & $\bar{G}$ & $\sigma$ & $x^{2}$ \\
\hline 52 & 140 & 0.0346 & 0.6700 & 6.761 & 9.910 & 7.80 & 3.161 & 1.26 \\
53 & 140 & 0.0351 & 0.6979 & 6.862 & 9.965 & 7.80 & 3.135 & 5.20 \\
54 & 140 & 0.0351 & 0.7831 & 6.540 & 10.099 & 7.31 & 3.076 & 6.07 \\
56 & 85 & 0.0361 & 0.6874 & 7.217 & 10.748 & 8.32 & 3.316 & 7.49 \\
57 & 85 & 0.0361 & 0.6781 & 7.186 & 10.710 & 8.32 & 3.321 & 5.63 \\
58 & 85 & 0.0361 & 0.7578 & 7.157 & 11.038 & 8.10 & 3.296 & 2.21 \\
\hline
\end{tabular}

$\operatorname{aI}_{G}=c_{2} \frac{C_{3}{ }^{G}}{G !} e^{-c_{3}}+\left(1-c_{2}\right) \frac{c_{4}^{G}}{G !} e^{-C_{4}}$ 


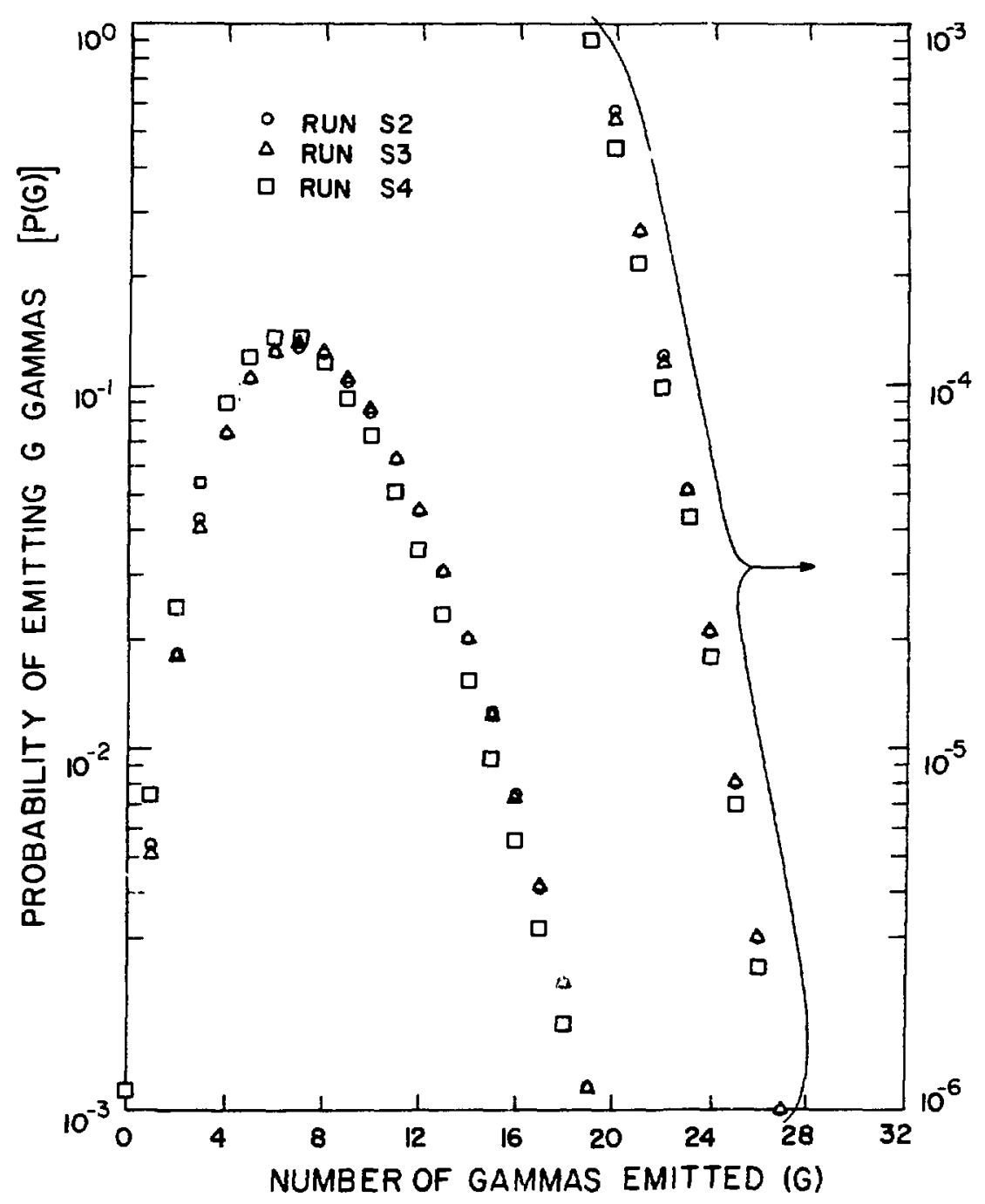

Fig. 12. Emission distribution inferred

from the data in Fig. 10 (140 keV threshold). 


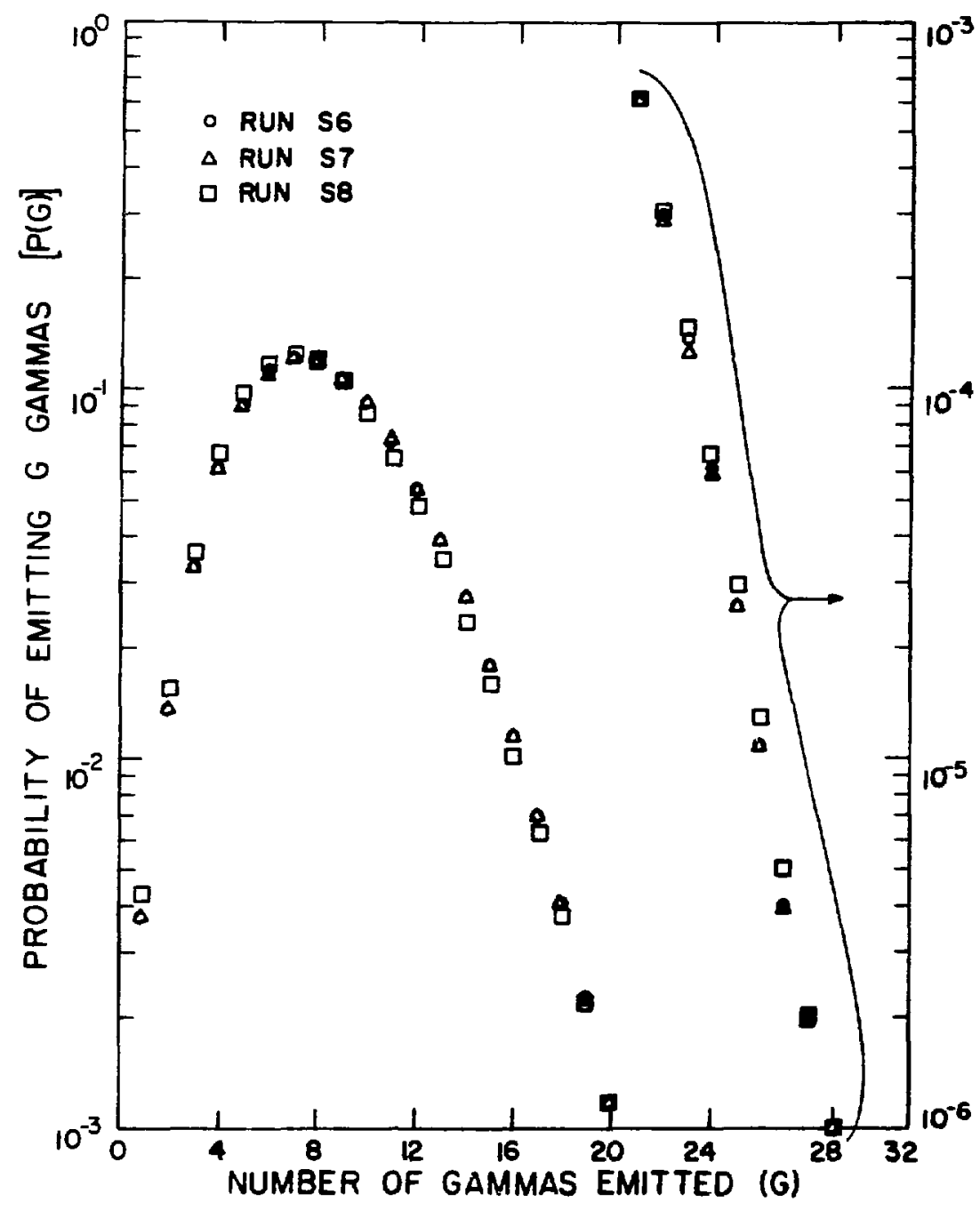

Fig. 13. Enission distribution inferred from the data in Fig. 11(85 keV threshold). 
Not only is the double Poisson the best model statistically, it is much easier to handle mathematically. Assume the model

$$
\pi(G)=C_{2} \frac{\left(C_{3}\right)^{G}}{G !} e^{-C_{3}}+\left(1-C_{2}\right) \frac{\left(C_{4}\right)^{G}}{G !} e^{-C_{4}}
$$

and combine with $P(G, M)$ to calculate $P(M)$, the probability of detecting an M-fold coincidence.

$$
\begin{aligned}
P(M) & =\sum_{G=M}^{\infty} \pi(G) P(G, M) \\
& =\sum_{G=M}^{\infty} C_{2} \frac{\left(C_{3}\right)^{G} e^{-C_{3}}}{G !} P(G, M)+ \\
& \sum_{G=M}^{\infty}\left(1-C_{2}\right) \frac{\left(C_{4}\right)^{G}}{G !} e^{-C_{4}} P(G, M)
\end{aligned}
$$

The two above summations are identical except for constants, so we will evaluate only the first one and insert different constants afterward to obtain the second term.

$$
P(M)=\sum_{G=0}^{\infty} C_{2} \frac{\left(C_{3}\right)^{G}}{G !} e^{-C_{3}} 3\left(\begin{array}{l}
N \\
M
\end{array}\right) \sum_{\ell=0}^{M}(-1)^{M-\ell}\left(\begin{array}{c}
M \\
\ell
\end{array}\right)[1-(N-l) E]^{G}+\cdot(i j)
$$

We have inserted the Van der Werf expression for $P(G, M)$ and set the lower limit on $G$ to 0 because $P(G, M)=0$ for $G<M$. Changing the order of summation and rearranging gives:

$$
P(M)=C_{2} e^{-C_{3}}\left(\begin{array}{l}
N \\
M
\end{array}\right)(-1)^{M} \sum_{\ell=0}^{M}(-1)^{\ell}\left(\begin{array}{l}
M \\
\ell
\end{array}\right) \sum_{G=0}^{\infty} \frac{C_{3}[1-(N-\ell) E]^{G}}{G !}
$$


The second summation is simply $e^{C_{3}[1-(N-l) \varepsilon]}$, which, when inserted yields:

$$
P(M)=C_{2}\left(\begin{array}{l}
N \\
M
\end{array}\right) e^{-C_{3} N}(-1)^{M} \sum_{l=0}^{M}(-1)^{l}\left(\begin{array}{c}
M \\
l
\end{array}\right) e^{C_{3} \varepsilon l}+\cdots
$$

The summation is just the binomial expansion of $\left(1-e^{C_{3} E}, M\right.$; taking into account the term $(-1)^{M}$, we have

$$
P(M)=\left(\begin{array}{l}
N \\
M
\end{array}\right) C_{2} e^{-C_{3} N \varepsilon}\left(e^{C_{3} \varepsilon}-1\right)^{M}+\left(1-C_{2}\right) e^{-C_{4} N \varepsilon}\left(e^{C_{4} \varepsilon}-1\right)^{M}
$$

where the second term has been obtained oy parallelism with the first. Because $C_{3}$ and $C_{4}$ are respectively the means of the two distributions

$$
\bar{G}=C_{2} C_{3}+\left(1-C_{2}\right) C_{4} \text {. }
$$

Figure 14 snows the agreement between inferred distributions for run S3 as obtained from two models, double Poisson and skewed pseudoGaussian. The agreement is very good between these two models.

At this point, we digress to describe some collateral tests made to verify the efficacy of the computer program. If we set $c_{2}=1$ in the above equation, we can calculate the distribution of detection multiplicities for a hypothetical poisson emission distribution of mean $\mathrm{C}_{3}$. This was done to obtain

$$
P(M) \text { for } 0 \leq M \leq 8 \text {. }
$$

These nine probabilities were then randomized to simulate the scatter that would be expected in a representative run of approximately $10^{7}$ events. We fed these artificial data into the fitting program. 


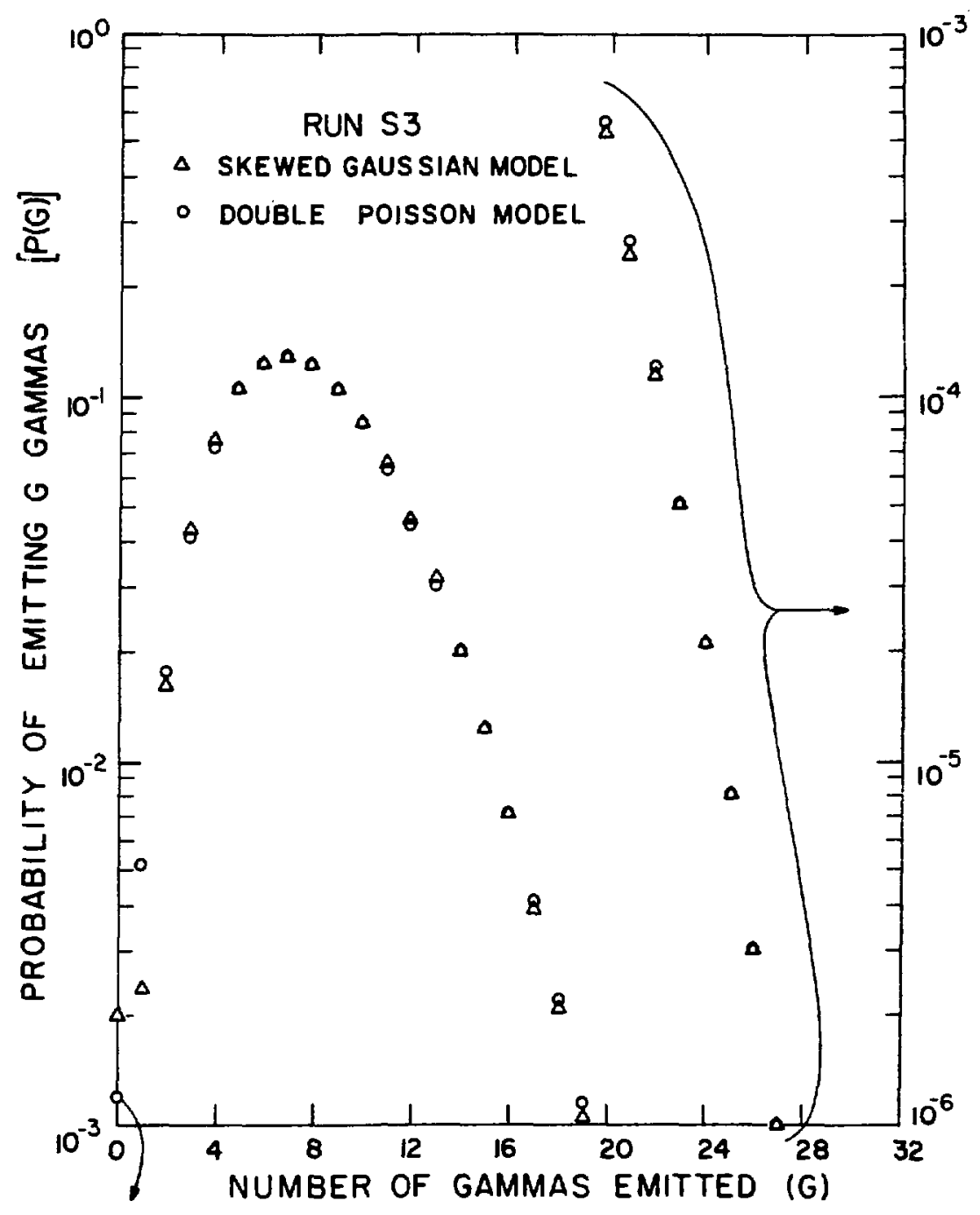

Fig. 14. Comparison of emission distributions for Run $\mathrm{S} 3$ as inferred from the double Poisson and skewed pseudo-Gaussian models. 
Fitting with the Poisson did, in fact, give us back the original emission distribution within reasonable statisties. More important, when the artificial data was fitted with the skewed pseudo-Gaussian model, the inferred emission distribution was about as close to the input distribution as was the result of the previous fit with the Poisson model.

\section{Energy}

It will be recalled from the discussion of the model fitting program that after the computation has converged the program calculates the following:

$\bar{H} \cdot(M)$ the average gamma impact multiplicity as a function of the detected multiplicity $M$.

$\bar{G}(M)$ the average emission multiplicity as a function of the detected multiplicity $M$.

The data collection program provides $\bar{E}$, which is the sum energy of $M$ triggered detectors divided by $M$. We then calculate $\bar{E} \cdot=\bar{E} \cdot \frac{M}{\bar{M}}$ to compensate for the fact that on the average some of the $M$ detectors will have experienced multiple impacts. Table 4 shows these results for the six sets of data. As seen in Figure 15, the average gama energy (in arbitrary units) decreases for increasing average emission multiplicity. In the cases of runs 54 and 58 in which the fission detector was biased toward fissions of high kinetic energy (that is, low excitation][1]], the effect is more pronounced. The dependence of individual gamma energy on gamma multiplicity is a very interesting phenomenon and we wish to examine the reliability of this observation with particular care. 
TABLE 4

DETERMINATION OF AVERAGE GAMMA ENERGY ASSOCIATED WITH MOST PROBABLE EMISSION NUMBER G( $\bar{M})$

\begin{tabular}{|c|c|c|c|c|c|c|c|c|c|c|c|c|c|}
\hline $\begin{array}{l}\text { Run } \\
\text { Number }\end{array}$ & $\begin{array}{c}\text { Threshold } \\
\text { keV }\end{array}$ & $\mathbf{M}^{\mathbf{A}}$ & $\bar{G}(M)^{b}$ & $\bar{M}^{\prime}(M)^{c}$ & $\tilde{E}(i d)^{d}$ & $\bar{E}^{i e}$ & $\underset{\text { Number }}{\text { Run }}$ & $\begin{array}{c}\text { Threshold } \\
\text { kev }\end{array}$ & $\boldsymbol{M}$ & $\bar{G}(M)^{b}$ & $\bar{M}^{\prime}(M)^{C}$ & $E(M)^{d}$ & $E^{\prime e}$ \\
\hline \$2 & 140 & $\begin{array}{l}1 \\
2 \\
3 \\
4 \\
5 \\
6 \\
7 \\
8\end{array}$ & $\begin{array}{r}6.58 \\
7.93 \\
9.32 \\
10.75 \\
12.17 \\
13.58 \\
14.94 \\
16.27\end{array}$ & $\begin{array}{l}1.136 \\
2.282 \\
3.441 \\
4.613 \\
5.796 \\
6.986 \\
8.180 \\
9.373\end{array}$ & $\begin{array}{l}56.64 \\
56.01 \\
55.56 \\
55.27 \\
55.09 \\
54.91 \\
55.62 \\
54.25\end{array}$ & $\begin{array}{l}49.86 \\
49.09 \\
48.44 \\
47.92 \\
47.52 \\
47.16 \\
47.60 \\
46.30\end{array}$ & S6 & 85 & $\begin{array}{l}1 \\
2 \\
3 \\
4 \\
5 \\
6 \\
7 \\
8\end{array}$ & $\begin{array}{r}6.80 \\
8.18 \\
9.63 \\
11.11 \\
12.61 \\
14.08 \\
15.52 \\
16.90\end{array}$ & $\begin{array}{l}1.150 \\
2.312 \\
3.490 \\
4.683 \\
5.893 \\
7.110 \\
8.333 \\
9.956\end{array}$ & $\begin{array}{l}51.93 \\
51.30 \\
50.83 \\
50.49 \\
50.35 \\
50.23 \\
50.27 \\
50.57\end{array}$ & $\begin{array}{l}45.16 \\
44.38 \\
43.69 \\
43.13 \\
42.72 \\
42.39 \\
42.23 \\
42.33\end{array}$ \\
\hline S3 & 140 & $\begin{array}{l}1 \\
2 \\
3 \\
4 \\
5 \\
6 \\
7 \\
8\end{array}$ & $\begin{array}{r}6.56 \\
7.89 \\
9.27 \\
10.68 \\
12.10 \\
13.51 \\
14.01 \\
16.24\end{array}$ & $\begin{array}{l}1.138 \\
2.286 \\
3.446 \\
4.618 \\
5.804 \\
6.996 \\
8.194 \\
9.393\end{array}$ & $\begin{array}{l}56.41 \\
55.79 \\
55.34 \\
55.04 \\
54.93 \\
54.88 \\
54.85 \\
55.40\end{array}$ & $\begin{array}{l}49.57 \\
48.81 \\
48.18 \\
47.67 \\
47.32 \\
47.87 \\
46.86 \\
47.18\end{array}$ & 57 & 85 & $\begin{array}{l}1 \\
2 \\
3 \\
4 \\
5 \\
6 \\
7 \\
8\end{array}$ & $\begin{array}{r}6.80 \\
8.19 \\
9.53 \\
11.12 \\
12.61 \\
14.08 \\
15.51 \\
16.88\end{array}$ & $\begin{array}{l}1.150 \\
2.312 \\
3.490 \\
4.684 \\
5.893 \\
7.109 \\
8.331 \\
9.553\end{array}$ & $\begin{array}{l}51.78 \\
51.18 \\
50.71 \\
50.44 \\
50.25 \\
50.07 \\
50.24 \\
49.26\end{array}$ & $\begin{array}{l}45.02 \\
44.27 \\
43.59 \\
43.82 \\
42.63 \\
42.26 \\
42.21 \\
41.25\end{array}$ \\
\hline $\begin{array}{l}54 \\
\text { As sume } \\
\varepsilon \text { constant }\end{array}$ & 140 & $\begin{array}{l}1 \\
2 \\
3 \\
4 \\
5 \\
6 \\
7 \\
8\end{array}$ & $\begin{array}{r}6.20 \\
7.53 \\
8.93 \\
10.40 \\
11.91 \\
13.41 \\
14.88 \\
16.29\end{array}$ & $\begin{array}{l}1.129 \\
2.268 \\
3.421 \\
4.591 \\
5.781 \\
6.982 \\
8.191 \\
9.401\end{array}$ & $\begin{array}{l}55.41 \\
54.42 \\
53.59 \\
52.94 \\
52.46 \\
52.26 \\
51.79 \\
48.86\end{array}$ & $\begin{array}{l}49.08 \\
47.99 \\
46.89 \\
46.12 \\
45.37 \\
44.90 \\
44.26 \\
41.58\end{array}$ & $\begin{array}{c}S 8 \\
\text { Assume } \\
\varepsilon \text { constant }\end{array}$ & $\begin{array}{r}85 \\
n t\end{array}$ & $\begin{array}{l}1 \\
2 \\
3 \\
4 \\
5 \\
6 \\
7 \\
8\end{array}$ & $\begin{array}{r}6.55 \\
8.01 \\
9.47 \\
10.99 \\
12.56 \\
14.11 \\
15.62 \\
17.07\end{array}$ & $\begin{array}{l}1.146 \\
2.303 \\
3.476 \\
4.669 \\
5.883 \\
7.110 \\
8.344 \\
9.580\end{array}$ & $\begin{array}{l}50.49 \\
49.44 \\
48.51 \\
47.91 \\
47.51 \\
47.15 \\
46.32 \\
46.93\end{array}$ & $\begin{array}{l}44.06 \\
42.93 \\
41.87 \\
41.05 \\
40.38 \\
39.79 \\
38.86 \\
39.19\end{array}$ \\
\hline
\end{tabular}

Detection multiplicity.

byost probeble emission number for M-fold detection.

CMost probable impact number for M-fold detection. d Average energy per detector in the $M$ triggered detector 5 .

Average energy per gamma $E^{\prime}=-\frac{M}{M^{\prime}(M)} \overline{E(M)}$ 


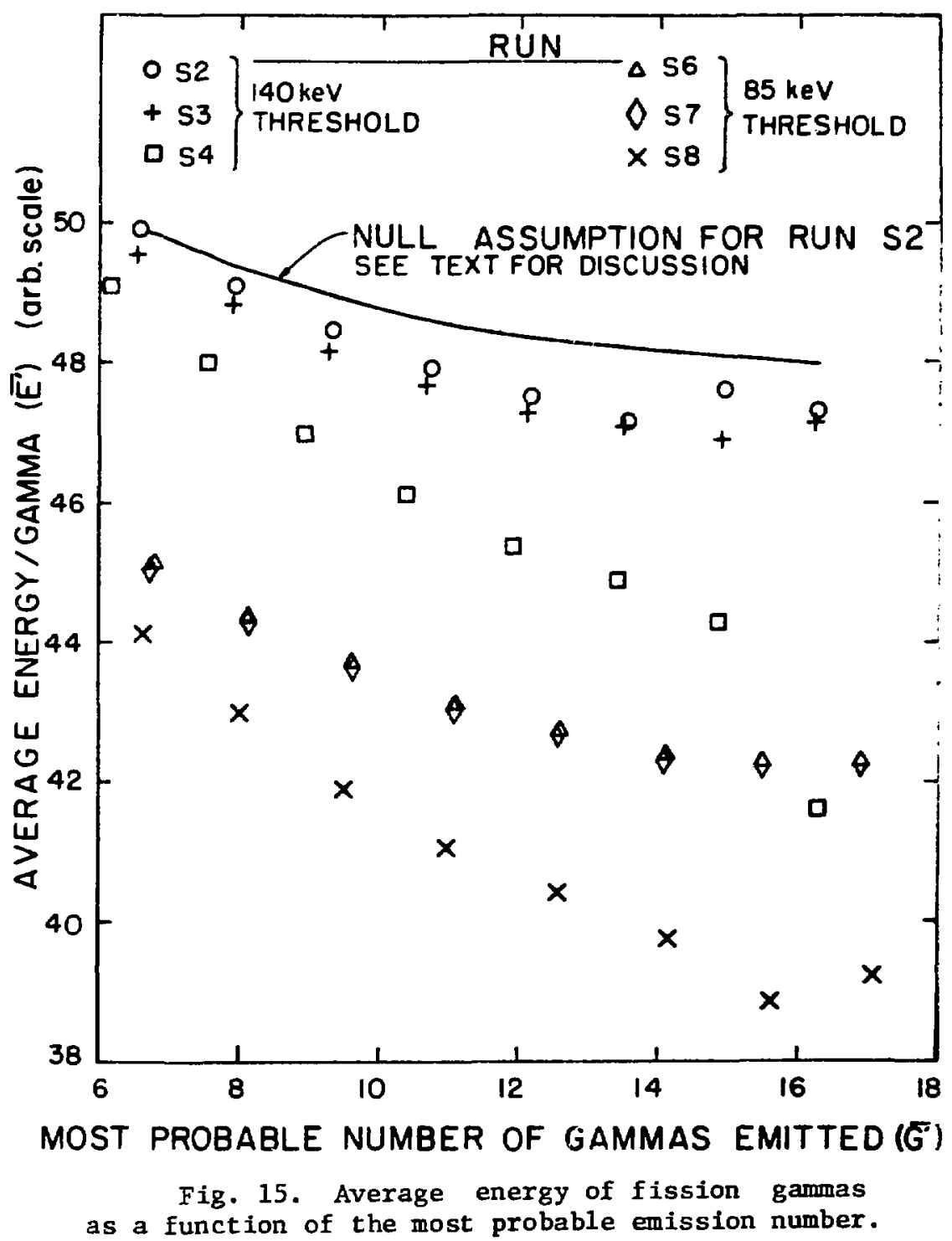


Is it real? For the moment, let us assume that it is not, that the average detected gamma energy is $E_{0}$ regardless of the emission multiplicity. In Table 2, we see that a substantial number of events under each multiplicity actually belong in the next lower multiplicity but have been "promoted" by a scattering. This might nave an effect on apparent energies somewhat like what has been observed in Figure 15. To be specific, let us examine the data for multiplicity 2 in Run 52 (Table 2). We see that for $2,782,169$ events of multiplicity 2 , an estimated 54,867 events belonged in multiplicity 1 .

At this point, we make two extremely conservative assumptions:

1. The scattered gamma can trigger the spurious count with zero energy input; tnat is, a single gamma which erroneously triggers a second detector still contributed only $E_{0}$ to the energy sum. This obviously ignores the minimum energy deposition required to trigger the second detector.

2. The erroneously counted gamma had an average energy to begin with. (obviously, a nigh energy gamma has a better chance of depositing enough energy to trigger the discriminator in the first detector and then scattering with enough remaining energy to trigger a second detector.)

Continuing with our assumptions, we estimate that the data acquisition program accumulated energy as follows for multiplicity 2:

From genuine 2-fold events:

$$
2 \times E_{0} \times(2,782,169-54,867)=5.4546 \cdot 10^{6} E_{0}
$$

From misplaced 1-fold events:

$$
1 \times E_{0} \times 54,867=\frac{0.0549 \cdot 10^{6} E_{0}}{5.5095 \cdot 10^{6} E_{0}}
$$


The data acquisition system assumes all 2-fold events are genuine and divides the energy sum by $2 \times 2,782,169$ to find that $\bar{E}(2)=0.990$ $E_{0}$. The solid line in Figure 15 shows the results of this kind of computation for all multiplicities in Run 52 . Despite the severity of the assumptions, the null hypothesis $\left(\bar{E}_{0}\right.$ constant) differs significantly from the actual data. We conclude that the effect is real. 
VII

\section{SOURCES OF BIAS}

There are several sources of spurious signals: for example, cosmic ray showers and fast neutrons. Occasionally, a genuine fission gamma will look like two fission gammas through a Compton scattering that enables it to trigger two separate detectors. Fast neutrons can easily cause scintillation signals in the hydrogenous plastic scintillators through proton recoils, but it is possible to discriminate against them by time of flight. The ${ }^{252} \mathrm{Cf}$ source foil is located at the center of the counter array. This gives a minimum flight distance of about $10 \mathrm{~cm}$ to the face of any of the gamma detectors; flight path to the edge of the detector face is around $11 \mathrm{~cm}$. Thus, even though they may penetrate some centimeters into the scintillator before interacting, the gammas traveling at $30 \mathrm{~cm} /$ nanosecond will interact, if at all, in less than approximately $2 / 3$ nanosecond. On the other hand, a 4-MeV neutron travels only $2.8 \mathrm{~cm} /$ nanosecond. There are few neutrons over $4 \mathrm{MeV}$. The estimated mean free path for a neutron in the scintillator is about $2 \mathrm{~cm}$. Taking this all into account, we can see that about 4-nanoseconds minimum flight time is required for a 4-MeV neutron and nearly 3 nanoseconds for an 8-MeV neutron. The time separation of the radiations can be seen in Figure 4. The channels are about 48 picoseconds wide. 
Aside from the fast neutrons, bias can be expected from the following causes:

1. In the fission detector:

a. False triggers from external causes such as cosmic rays or environmental radiation $\left(e . g .40_{K}\right)$.

b. False triggers from internal causes such as alphas, betas, neutrons, or nonfission gammas emitted by the $252 \mathrm{Cf}$ source.

2. In the eight gamma detectors:

a. False counts from exiernal causes such as cosmic rays or environmental radiation $\left(e \cdot g .40_{K}\right)$.

b. False counts from nonfission gammas emitted by the fission products in the source or from actual fission gammas that happen not to originate in the trigger fission.

c. False multiplicities arising from complex scattering events in the detectors or adjacent structure.

3. Simplification for convenience in analysis:

a. Assumption that all gamma detectors have same detection efficiency.

b. Assumption that gammas are detected with same efficienoy regardless of the multiplicity of the event.

We will take these in turn and discuss the experiments or analysis used to determine their influence on experimental results.

To evaluate la above, we removed the ${ }^{252} \mathrm{Cf}$ source and operated the otherwise normal experiment during two overnight runs for a total of about 30 hours. Not a single trigger was observed. This is surprising at first but plausible after some consideration. The scintillator on the fission detector is only $1 / 4 \mathrm{~mm}$ thick. Moreover, the detector is operated at such a high threshold as to reject approximately $6 \mathrm{MeV}$ alphas and approximately $70 \mathrm{MeV}$ fission fragments. Even 
if a suitably large energy were transferred to an electron in the scintillator, the mean free path for an electron of such energy is so long that most of the energy would be lost from the scintillator. We can ignore false triggers from this source.

Internal false triggers may occur due to alpha pile-up. In view of the very high threshold, no other source seems plausible. We can estimate an upper limit on the rate of occurrence. The total alpha emission from the ${ }^{252} \mathrm{Cf}$ is $24,000 \mathrm{sec}^{-1}$, of which one-half, or less, impinge directly on the scintillator. Because of the extremely narrow pulses in the Pilot $U$ scintillator and associated electronics, two pulses must occur within less than approximately 5 nanoseconds for effective pile-up to occur. These numbers lead to an estimate of approximately 0.7 alpha pile-ups per second, or less than one false trigger in a thousand.

A simple measurement was sufficient to evaluate the combined background from items $2 a$ and $2 b$ above. They are both simply counts from events not related to the trigger event. We substituted a signal from an electronic pulse generator for the trigger signal from the fission detector. Everything else was left exactly as for the actual experiment; the ${ }^{252} \mathrm{Cf}$ source remained in its normal position. With the gamma threshold set at $85 \mathrm{keV}$ (the lower of the two used), we counted (in all eight detectors combined) about one event in $10^{4}$ gates. This is a reflection of the stringency of the timing criterion and is small enougn to be neglected. 
The most important source of error was the scattering effect of real fission gammas ( $2 c$ above). A Compton scattering in one detector can produce a scattered gamma capable of triggering another detector. Early experiments indicated that was happening with statistically detectable frequency between adjacent detectors. We have previously described the snielding used to minimize this problem. However, it is geometrically impossible to shield nonadjacent detectors from each other without obstructing the detector's "view" of the source.

Another potential source of spurious coincidences is pair production in the lead shielding or other structure. The simultaneous annihilation gammas might also cause a spurious coincidence. Measurements of the gamma spectrum associated with fission show, as would be expected, lead $x$-rays (at $72.8,75.0,84.3$, and $87.3 \mathrm{keV}$ ) in coincidence with fission; hence these must have been created by genuine fission gammas. Do these $x$ rays constitute a source of false coincidences? It is barely possible that the last two might trigger a detector at the lower of the two thresholds used $(85 \mathrm{keV})$; if the parent gamma happened to trigger another detector, then the multiplicity would be erroneously enhanced ("promoted") by the spurious additional count.

Fortunately, there were simple ancillary experiments to measure the extent to which the californium data is biased by the above processes. We removed the ${ }^{252} \mathrm{Cf}$ source from the fission detector and substituted a source of ${ }^{54} \mathrm{Mn}$. This isotope decays by electron capture and emits a single gamma of $835 \mathrm{keV}$ (no betas). We increased the gain and decreased the threshold in the fission detector so that 
almost any scattering event in the scintillator would trigger an analysis cycle. A second, subsidiary experiment showed that the discriminator was in fact so low that further lowering of the threshold had very small effect on count rate and measured gamma-ray energy. This confirms the above assertion concerning "almost any scattering event."

The experiment was then operated in a normal fashion. Each countable event began with a scattering in the fission detector scintillator. The scattered gamma might then strike one of the eight gamma detectors and be recorded as a multiplicty of 1 . Occasionally, the gamma might be re-scattered and trigger one of the other seven detectors, registering a multiplicity of 2. Because the source emits a single gamma, the only way, ignoring background, to observe multiplicity 2 is by means of such a multiple scattering.

If we divide the number of times multiplicity 2 was observeu $b_{y}$ the number of times multiplicity 1 or 2 was observed, we have a direct measurement of the probability that a scattered gamma from one detector will trigger one of the other seven detectors. Division by 7 then gives the average probability that the scattered gamma will trigger a specified one of the other detectors.

The experiment was then repeated with a ${ }^{60}$ Co source. Cobalt-60 emits (within approximately 1 picoseconds of each other) two gammas of 1170 and $1330 \mathrm{keV}$. For the purpose of this experiment, we take them both as $1250 \mathrm{keV}$. Cobalt-50 also emits betas, but these were suppressed with a cover of polyeihylene. With this source, we may expect to observe a substantial number of events with a genuine multiplicity 2, and occasionally there will be an event of multiplicity 3 caused by 
a scattering as already described. We divide the number of times multiplicity 3 was ooserved by the number of times multiplicity 2 or 3 was observed. This gives us the probability that one of the two detectors with genuine events scatters a countable gamma into one of the other six detectors. Dividing this probability by 12 (the number of combinations), we obtain tne probability of scattering from one detector to another specified detector.

This is straightforward for the two sources, but the results must be adjusted for energy dependence before they can be used to correct counting data from ${ }^{252} \mathrm{Cf}$ in the experiment proper. The basis for correction is contained in the data from each run. Each operation of the experiment yields an average energy (on an arbitrary scale) for the events counted in the eight gamma detectors. The energies for ${ }^{252}$ Cf lie conveniently between those for ${ }^{54} \mathrm{Mn}$ and ${ }^{60} \mathrm{Co}$, so we can get the desired correction probability by simple interpolation. The above described procedure to obtain the corrections probabilities for ${ }^{252} \mathrm{Cf}$ is demonstrated in Table 5 .

We restate for the sake of clarity, the "false count probability" estimated for ${ }^{252} \mathrm{Cf}$ is the probability that a genuine fission gamma event in one detector will be associated with a spurious count by scattering into another specified detector.

These probabilities are not small; corrections were made by iteration. Suppose $\mathrm{n}_{3}$ genuine 3-fold coincidences occurred. There are 15 ways that a scattering event could falsely "promote" a 3-fold to a 4-fold coincidence (scattering from any of the 3 properly triggered detectors to any of the 5 detectors that should not have been 
TABLE 5

DETERMINATION OF FALSE COUNT PROBABILITY

\begin{tabular}{|c|c|c|c|c|c|c|}
\hline \multirow{2}{*}{$\begin{array}{c}\text { Threshold } \\
\text { (keV) }\end{array}$} & \multicolumn{2}{|c|}{$54 \mathrm{~m}$} & \multicolumn{2}{|c|}{6000} & \multicolumn{2}{|c|}{$252 \mathrm{Cf}$} \\
\hline & 85 & 140 & 85 & 140 & 85 & 140 \\
\hline $\begin{array}{l}\text { Counting } \\
\text { cime (sec) }\end{array}$ & $3,276.8$ & $3,276.8$ & $3,276.8$ & $3,276.8$ & & \\
\hline $\begin{array}{l}\text { Number of } \\
\text { singles }\end{array}$ & $\begin{array}{c}470,518 \\
(470,423)^{a}\end{array}$ & $\begin{array}{c}399,492 \\
(399,403)\end{array}$ & - & - & - & - \\
\hline $\begin{array}{l}\text { Number of } \\
\text { doubles }\end{array}$ & $\begin{array}{c}17,000 \\
(16,951)\end{array}$ & $\begin{array}{c}7,743 \\
(7,696)\end{array}$ & $\begin{array}{c}156,625 \\
(156,576)\end{array}$ & $\begin{array}{c}127,499 \\
(127,452)\end{array}$ & - & - \\
\hline $\begin{array}{l}\text { Number of } \\
\text { triples }\end{array}$ & - & - & $\begin{array}{c}8,844 \\
(8,834)\end{array}$ & $\begin{array}{c}4,738 \\
(4,728)\end{array}$ & - & - \\
\hline $\begin{array}{l}\text { Fraction } \\
\text { of false } \\
\text { doubles }{ }^{b}\end{array}$ & 0.03478 & 0.01890 & - & - & - & - \\
\hline $\begin{array}{l}\text { Fraction } \\
\text { of false } \\
\text { triples }\end{array}$ & - & - & 0.0534 & 0.03577 & - & - \\
\hline $\begin{array}{l}\text { False coumt } \\
\text { probabilityd }\end{array}$ & 0.0050 & 0.0027 & 0.0044 & 0.0030 & $(0.0045)^{e}$ & $(0.0029)^{\mathrm{e}}$ \\
\hline $\begin{array}{l}\text { Energy } \\
\text { (arbitrary } \\
\text { units) }\end{array}$ & 24.8 & 28.1 & 48.4 & 52.8 & 43.5 & 48.5 \\
\hline
\end{tabular}

acount corrected for background in parentheses.

b(Net doubles)/(Nht singles plus net doubles).

C(Net triples)/(Net doubles plus net triples).

din a specified detector.

Inferred probability of a false count in a specified detector obtained by interpolation on energy. 
triggered.) For example, if the discriminators were set at $140 \mathrm{keV}$, we would estimate that a certain number, $Q=15 \times 0.0029 \times \mathrm{n}_{3}$, of the 3-fold multiplicites have been erroneously counted as 4-fold multiplicities. Because we do not know $n_{3}$, the number of genuine 3-fold events, we use $n$ ' 3 ' the number of observed 3-fold events, as a reasonable estimate of $n_{3}$. We calculate $Q^{\prime}=15 \times 0.0029 \times n^{\prime}{ }_{3}$ and "retrieve" $Q$ ' events from the number of 4 -fold events cbserved. That is, we diminish the 4-fold count by $Q^{\prime}$ and increase the 3-fold count by the same number. This is done sequentially for all multiplicities except 0 multiplicity, which, of course, remains unchanged. After four iterations, we nave a stable set of numbers $n_{M}$, which is the best estimate of the true distribution we would have observed if there had been no intercommunication between detectors. The extent of these corrections is shown in Table 2.

For convenience in analysis, it was assumed that all the gamma detectors had the same efficiency (item $3 a$ above). In reality, the efficiencies varied up to $7 \%$ from the mean. Van de Werf[25] has also derived a general form of Eq. 5 for coincidences between detectors of unequal efficiency. For the purpose of examining this question, the probabilities of the eight detection multiplicities were calculated for a Poisson emission distribution under two assumptions:

1. Equal efficiencies for all gamma detectors (using Eq. 5)

2. Actual unequal efficiencies (using the general equation) Consider the differences between the actual efficiencies and the average efficiency as a set of eight perturbations on the average efficiency. To first order in these perturbations, there is no difference 
between 1 and 2 above. To second order, the differences in calculated detection multiplicities were all less than $1 / 3 \%$. This was quite small compared with the corrections made for scattering (Table 2), and no corrections were made for this effect.

The average gamma energy varies with the multiplicity, so tine detection efficiency must also vary with multiplicity. In applying Eq. 5, we tacitly assume efficiency does not depend on multiplicity (item 3b on page 60). Measurements with ${ }^{60} \mathrm{Co}$ and ${ }^{54} \mathrm{Mn}$ imply that the efficiency changes very little in the narrow energy region containing all the average energies associated with the various multiplicities. In the worst case, Run $S 8$, the spread is only approximately 15\%, and typically is much smaller. Based on the cobalt and manganese source measurements, we estimate a maximum difference in efficiency of 2\% between high and low multiplicity events. The effect of this assumed difference can be estimated from Eq. 20. The term with $\mathrm{C}_{4}$ in it contributes almost all of the probability of high multiplicity events, while the term with $\mathrm{C}_{3}$ contributes almost all of the probability of low multiplicity events. If we assume the efficiencies in the two terms differ by $2 \%$, we can estimate the effect on $c_{3}$ and $C_{4}$, given that the fitting process holds the left hand side constant at the experimental value. This leads to an estimate of $11 / 3 \%$ uncertainty for $C_{3}$ and $2 / 3 \%$ for $C_{4}$ (still considering Run $S 8$ ). The uncertainties for Run $\$ 4$ are about the same. For the remaining runs, they are reduced by about one half. 
The foregoing work leads to the following mathematical models for the multiplicity of gammas emitted in the spontaneous fission of ${ }^{252} \mathrm{Cf}$ :

For gamma threshold at $140 \mathrm{keV}$ :

$$
\pi(G)=0.675 \frac{(6.78)^{G} e^{-6.78}}{G !}+0.325 \frac{(9.92)^{G} e^{-9.92}}{G !}
$$

$$
(G \geq 0)
$$

For gamma threshold at $85 \mathrm{keV}$ :

$$
\pi(G)=0.682 \frac{(7.20)^{G} e^{-7.20}}{G !}+0.318 \frac{(10.71)^{G} e^{-10.72}}{G !}
$$

$$
(G \geq 0)
$$

No fundamental physical significance is attributed to these models. They are simply mathematical representations judged most suitable by the $\chi^{2}$ criterion.

There is a marked decrease in average gamma energy with increasing multiplicity. Nevertheless, the total gamma energy is greater for higher multiplicity; that is, the decrease in individual gamma energy is not large enough to offset the increase in the number of gammas.

An attempt was made to correlate neutron multiplicity with gamma multiplicity. A second 16-channel coincidence register was separately gated to observe discriminator pulses from the gamma detectors in the time window appropriate to the arrival of fast neutrons. The data acquisition program was adapted from the one described earlier. 
Preliminary runs were made and the system functioned as described. The problem arises in the discriminator deadtime (nominally 9-1/2 nanoseconds). If a detector detects a gamma, the deadtime in that detector channel extends well into the time window in which neutrons snould be detected. As a result, the deadtime corrections were large and could not be estimated with any degree of confidence. The data appeared not worth further analysis.

The correlation of gamma multiplicity with neutron multiplicity could be measured effectively by a fairly simple extension of the present experiment. If the entire gamma detector array in Figure 2 were enclosed in a so-called "barrel counter" (a large cavity neutron counter capable of accepting a full-sized 55-gallon drum), it would be possible with small software changes to count the neutrons after thermalization in the barrel counter walls. This would permit correlated measurements of gammas and neutrons, although it might be anticipated that the results would confirm those of Nifenecker.[11]

An attempt was also made to discover if any gamma energies were preferentially associated with low, moderate or high emission multiplicities. This required the addition of a lithium-drifted germanium detector (Geli) to the detector array and substantial modification of the data acquisition program. After each fission event in the fission detector, the Geli detector was queried. If it had detected a gamma, then the multiplicity detected by the eight plastic detectors was determined. Depending on whether the multiplicity was "low" (0-1), "moderate" (2-4), or "high" (5-8), the pulse height determined by the Geli was stored in one of three 4096-channel spectra. 
Here are some tentative results as an indication of the possibilities of this line of research. There were a total of nearly 100 resolvable gamma energies. These included energies agreeing with many of the 24 listed by Rumpold[8] and the 31 listed by Bowman ex al.[24] (Tnese two references do not find many lines in common.) The following gamma energies snow preferential emission:

\begin{tabular}{|c|c|}
\hline $\begin{array}{c}\text { With Low } \\
\text { Multiplicity } \\
\text { (kev) }\end{array}$ & $\begin{array}{c}\text { With High } \\
\text { Multiplicity } \\
\text { (kev) } \\
\end{array}$ \\
\hline $\begin{array}{l}145.2 \\
147.8 \\
150.0\end{array}$ & $\begin{array}{l}454.5 \\
470.6 \\
475.1\end{array}$ \\
\hline $\begin{array}{l}165.4 \\
166.9 \\
290.1\end{array}$ & $\begin{array}{l}572.7 \\
578.5 \\
585.9\end{array}$ \\
\hline 329.4 & \\
\hline 592.1 & \\
\hline
\end{tabular}

The above results come from a single 5-day run. This work requires handling large masses of data and deserves a separate, sustained exploration because of the potential value to the interpretation of nuclear structure. 
APPENDIX A

POSSIBLE MODELS FOR GAMMA EMISSION DISTRIBUTION

We have tested some twenty different analytical models. Their inclusion depends to a varying extent on mathematical convenience and plausibility with respect to the fission process. Here are the principal candidates to represent the multiplicity distribution of prompt fission gammas:

1. Poisson distribution

$$
\pi(n)=\frac{(\bar{n})^{n} e^{-\bar{n}}}{n !}
$$

This is mathematically the most convenient; its applicability is doubtful because it assumes independent events. The emission of gammas from a single fission cannot be considered independent because they draw on the same store of excitation energy.

2. Double Poisson

$$
\pi(n)=A \frac{\left(\bar{n}_{1}\right)^{n} e^{-n_{1}}}{n !}+(1-A) \frac{\left(\bar{n}_{2}\right)^{n} e^{-n_{2}}}{n !}
$$

This was suggested by Gordon Hansen as having more flexibility (3 parameters) while still being analytically convenient.

3. Pseudo-Gaussian distribution

$$
\pi(n)=c_{1} e^{-c_{2}(n-\bar{n})^{2}}
$$


This is termed pseudo-Gaussian because it adapts the Gaussian form to a discrete variable, the number of gammas emitted. This is a reasonable possibility because nature sometimes works this way.

4. Skewed pseudo-Gaussian

$$
\begin{aligned}
& \pi(n)=c_{1}(n)^{C_{3}} e^{-c_{2}\left(n-\bar{c}_{4}\right)^{2}} \quad(n \geq 1) \\
& \pi(0)=c_{5} e^{-c_{2}\left(-c_{4}\right)^{2}}
\end{aligned}
$$

This distribution provides more parameters to allow more flexibility in fitting. From a practical standpoint, setting $\pi(0)$ free makes it possible to accommodate errors which might arise from even a small fraction of false fission triggers.

5. Pseudo-Cauchy

$$
\pi(n)=\frac{C_{1}}{C_{2}+(n-\bar{n})^{2}}
$$

Nature sometimes acts this way, as for example, in the density of nuclear resonance levels.

5. Skewed pseudo-Cauchy

$$
\begin{aligned}
& \pi(n)=\frac{c_{1}(n)^{C_{3}}}{c_{2}+(n-\bar{n})^{2}} \quad(n \geq 1) \\
& \pi(0)=c_{4}
\end{aligned}
$$

This is simply an effort to add more flexibility to the foregoing. 
7. Pseudo-lognormal

$$
\pi(n)=\frac{C_{1} e^{-C_{2}\left(\ln N-C_{3}\right)^{2}}}{n}
$$

This is a little farfetched since the lognormal is more often associated with economic or biological distributions. However, it has been applied to the distribution of fragment sizes after a rock crushing operation.

8. A large number of very simple models were tried in the beginning and quickly discarded. They included:

a. Level distributions of varying lengths.

b. Ramp distributions of both positive and negative slope and varying lengths.

9. Finally, there was something termed a "broken stick" distribution for want of a better name. In the Hoffman paper, [7] we see that the total gamma energy in a single fission is roughly equal to the energy required to boil of one prompt neutron (approximately $7 \mathrm{MeV})$.

This leads to the following conjecture: suppose we visualize the total excitation energy of the fission fragments as a stick some 30 or so units (MeV) long. Suppose further that the fission divides the energy (breaks the stick) at some statistically distributed point. The fission fragments then de-excite by emitting prompt neutrons so long as the excitation energy is greater than that necessary to emit a neutron. Thereafter, gammas are emitted. On the average, the energy "left over" for gammas in each fission fragment would amount to about 
one nalf that necessary to emit a neutron, so this would give a total gamma yield equivalent to that required for one neutron. This assumption leads to a distribution in the approximate form of an inverted $V$ (ramp up, ramp down). It was soon found to be a poor candidate and was discarded. 


\section{APPENDIX B}

VAN DER WERF'S EQUATION

With the permission of the author, [25] we reproduce here (with ' different symbols) his derivation of the equation used in calculating the number (M) of detectors triggered by a shower of $G$ gammas on an array of $N$ identical counters of efficiency $\varepsilon$.

$$
\begin{gathered}
P_{N}(G, M)=\text { Prob. }\left\{M^{\prime} \text { gammas hit somewhere in the } N \text { detectors }\right\} \\
x \text { Prob. }\left\{M^{\prime} \text { gammas are distributed in exactly } M\right. \\
\text { detectors. }\}
\end{gathered}
$$

The first probability is simply the binomial distribution of hits and misses where the probability of a hit is $\mathrm{Ne}$.

Prob \{that $N$ detectors are hit by $M^{\prime}$ gammas =

$$
\left(\begin{array}{l}
G \\
M^{\prime}
\end{array}\right)(N E)^{M^{\prime}}(1-N \varepsilon)^{G-M^{\prime}}
$$

The second probability crimesponds to that of finding exactly $M$ boxes occupied if $M^{\prime}$ objects are distributed over $\mathbf{N}$ boxes.

Prob \{M' gammas are distributed in exactly $M$ detectors $\}=$

$$
\left(\begin{array}{l}
N \\
M
\end{array}\right) \frac{M !}{N^{\prime}} \quad d_{M^{\prime}}^{(M)}
$$

\footnotetext{
(M)

$\mathscr{d}_{M}$, is Stirling's number of the second kind, which gives the number of ways $M$ ' objects can be partitioned into $M$ nonempty subsets. In terms of a physical model, it is the number of ways $M$ ' gammas can
} 
trigger $M$ interchangeable detectors. To calculate the total number of ways that $M^{\prime}$ gammas can trigger $M$ detectors out of an $N$-detector array, the Stirling number is multiplied by:

1. $M$ ! to account for the fact that the detectors are identifiable

2. $\left(\begin{array}{l}N \\ M\end{array}\right)$ to account for the number of ways that $M$ detectors can be chosen from the $\mathrm{N}$ detectors in the array

When this product is divided by the total number of ways that the $M$ ' gammas can impinge on $N$ detectors $\left(N^{M^{\prime}}\right)$, we have an analytical expression (Eq. 33) for the second probability in Eq. 3l. Multiplying the two probabilities, we have:

$$
P_{N}(G, M)=\sum_{M^{\prime}=M^{\prime}}^{G}\left(\begin{array}{l}
G \\
M^{\prime}
\end{array}\right) \varepsilon^{M^{\prime}}(1-M E)^{G-M^{\prime}}\left(\begin{array}{l}
N \\
M
\end{array}\right) M ! \mathbb{M}_{M^{\prime}}^{(M)}
$$

Explicitly,

$$
{\stackrel{(}{M^{\prime}}}^{(M)}=\frac{1}{M !} \sum_{\ell=0}^{M}(-1)^{M-\ell}\left(\begin{array}{c}
M \\
\ell
\end{array}\right) \ell^{M \cdot}
$$

Because

$$
\mathscr{\mathcal { E }}_{M^{\prime}}^{(M)}=0 \text { for } M^{\prime}<M
$$

the two expressions can be combined and the summation allowed to run from $M^{\prime}=0$ instead of $M^{\prime}=M$. Rearranging, we have

$$
P_{N}(G, M)=\sum_{\ell=0}^{i 1}(-1)^{M-\ell}\left(\begin{array}{c}
M \\
\ell
\end{array}\right)\left(\begin{array}{l}
N \\
M
\end{array}\right)\left[\sum_{M^{\prime}=0}^{G}\left(\begin{array}{c}
G \\
M
\end{array}\right)(\ell \varepsilon)^{M^{\prime}}(1-N \varepsilon)^{G-M^{\prime}}\right]
$$


The term in brackets is the binomial expansion of

$[1-(N-\ell) E]^{G}$ so

$$
P_{N}(G, M)=\left(\begin{array}{c}
N \\
M
\end{array}\right) \sum_{\ell=0}^{M}(-1)^{M-\ell}\left(\begin{array}{c}
M \\
\ell
\end{array}\right)[1-(N-\ell) \varepsilon]^{G}
$$

which is the equation we have used to infer the emission distribution of gammas.

Equation 36 can be rearranged to express the probability tnat from a shower of $G$ gammas on $N$ detectors, exactly $M^{\prime}$ of those gammas trigger exactly $M$ detectors.

$$
P_{N}\left(G, M^{\prime}, M\right)=\left(\begin{array}{l}
N \\
M
\end{array}\right)\left(\begin{array}{l}
G \\
M^{\prime}
\end{array}\right)(1-N \varepsilon)^{G-M^{\prime}} \sum_{\ell=0}^{M}(-1)^{M-l}\left(\begin{array}{l}
M \\
\ell
\end{array}\right)(\ell \in)^{M^{\prime}}
$$

This equation we have used to calculate the average impact multiplicity $\overline{1}$. 


\section{APPENDIX C \\ FLOW CHARTS AND PROGRAM LISTINGS FOR THE \\ DATA ACQUISITION PROGRAM}

The experiment acquired data by means of two mutually dependent computer programs in the LeCroy 3500. A FORTRAN program, GEN, accepted keybr.urd commands to set a count time and begin data acquisition. This it did by calling an assembly language subprogram, ACQUl, contained in a listing file named GENMAC. ACQUl then controlled all data acquisition until expiration of the specified counting time. Control then returned to $\mathrm{GEN}$, which affcrded the operator a number of options as to what data would be in the output and in what form.

In this appendix, the flow charts are given first and then the listings for the above programs. 


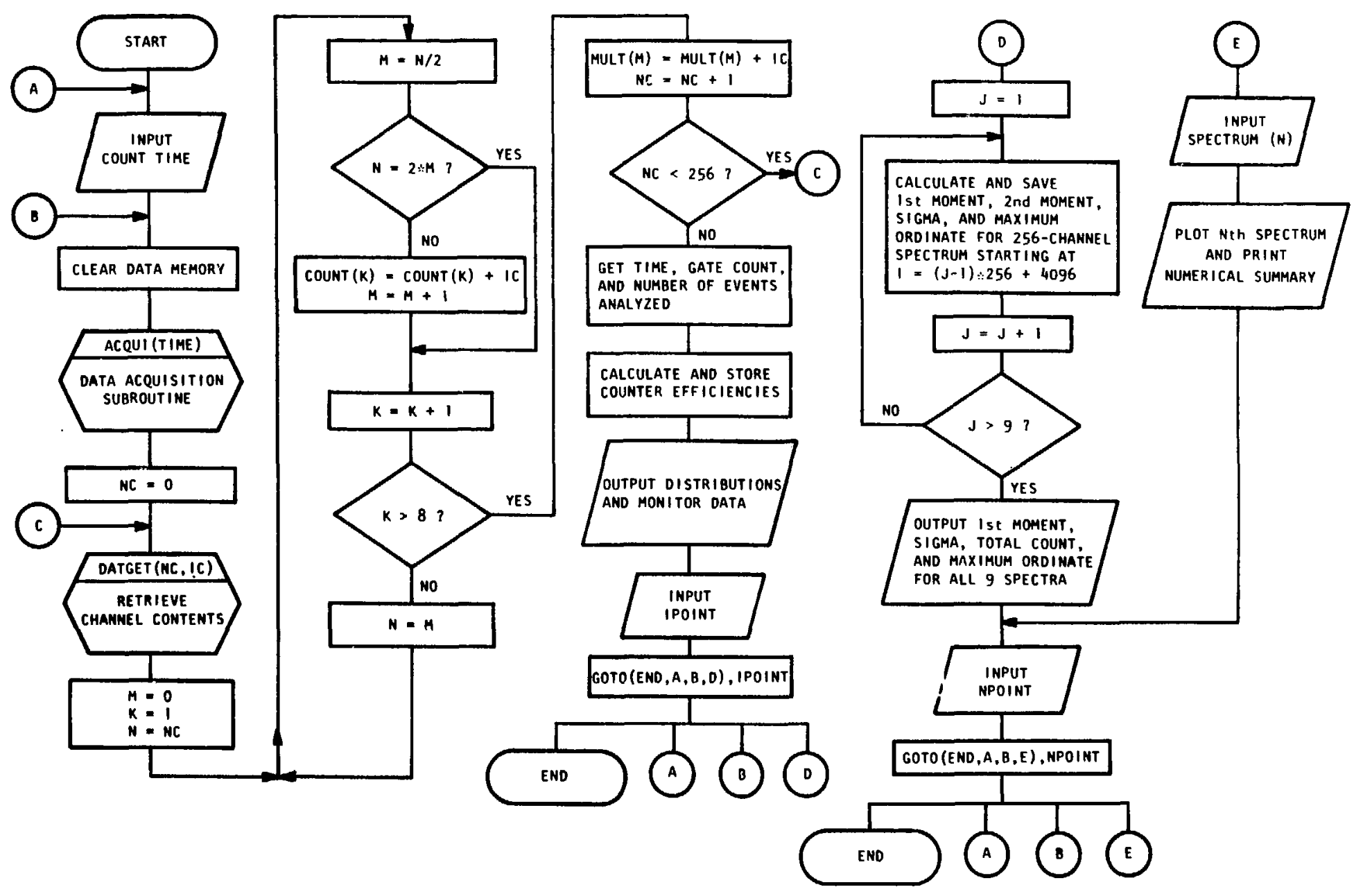

Fig. 16. Flow chart for the main data acquisition program. 


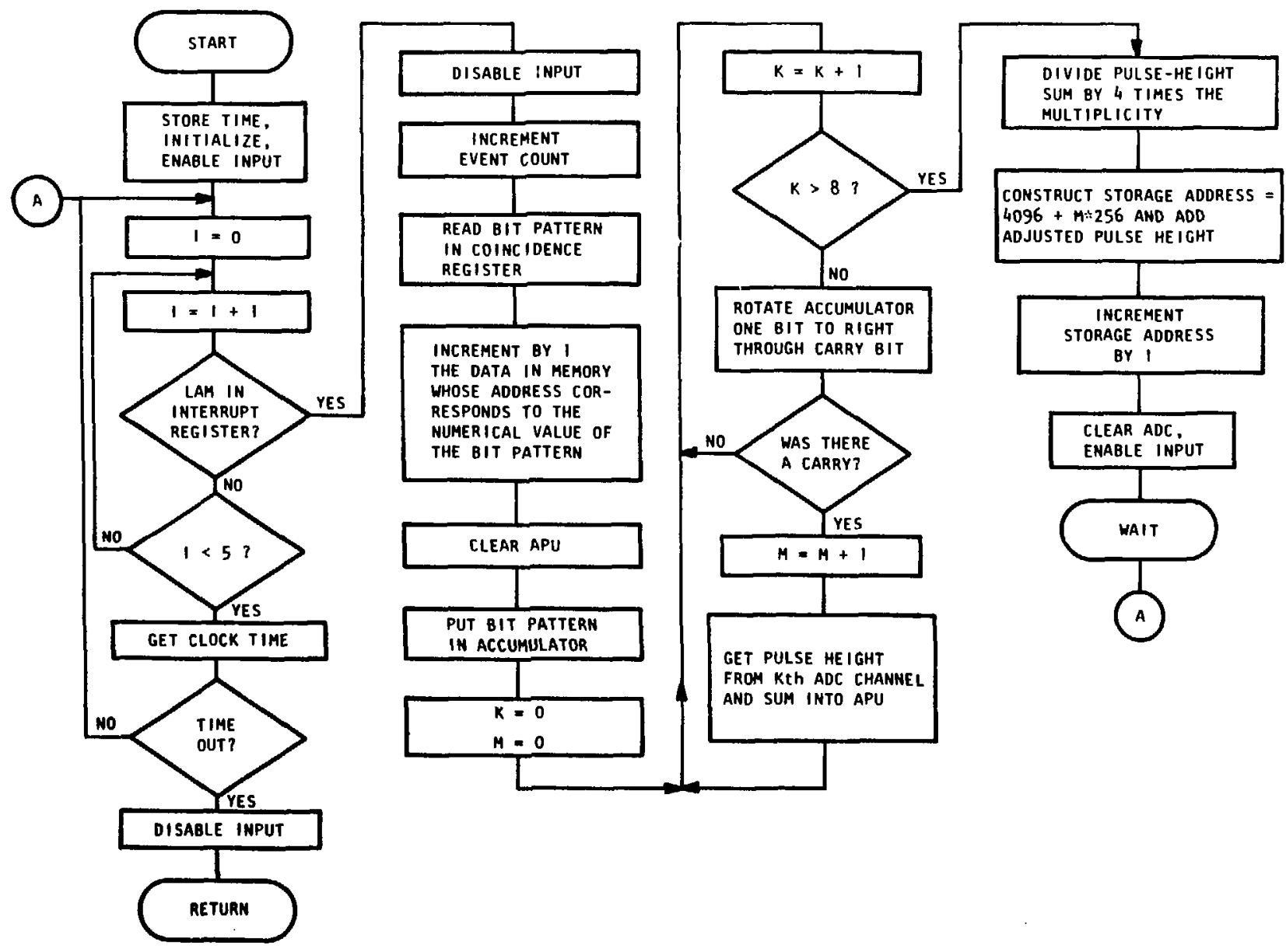

Fig. 17. Flow chart for the assembly language subroutine called by the main program to acquire data. 


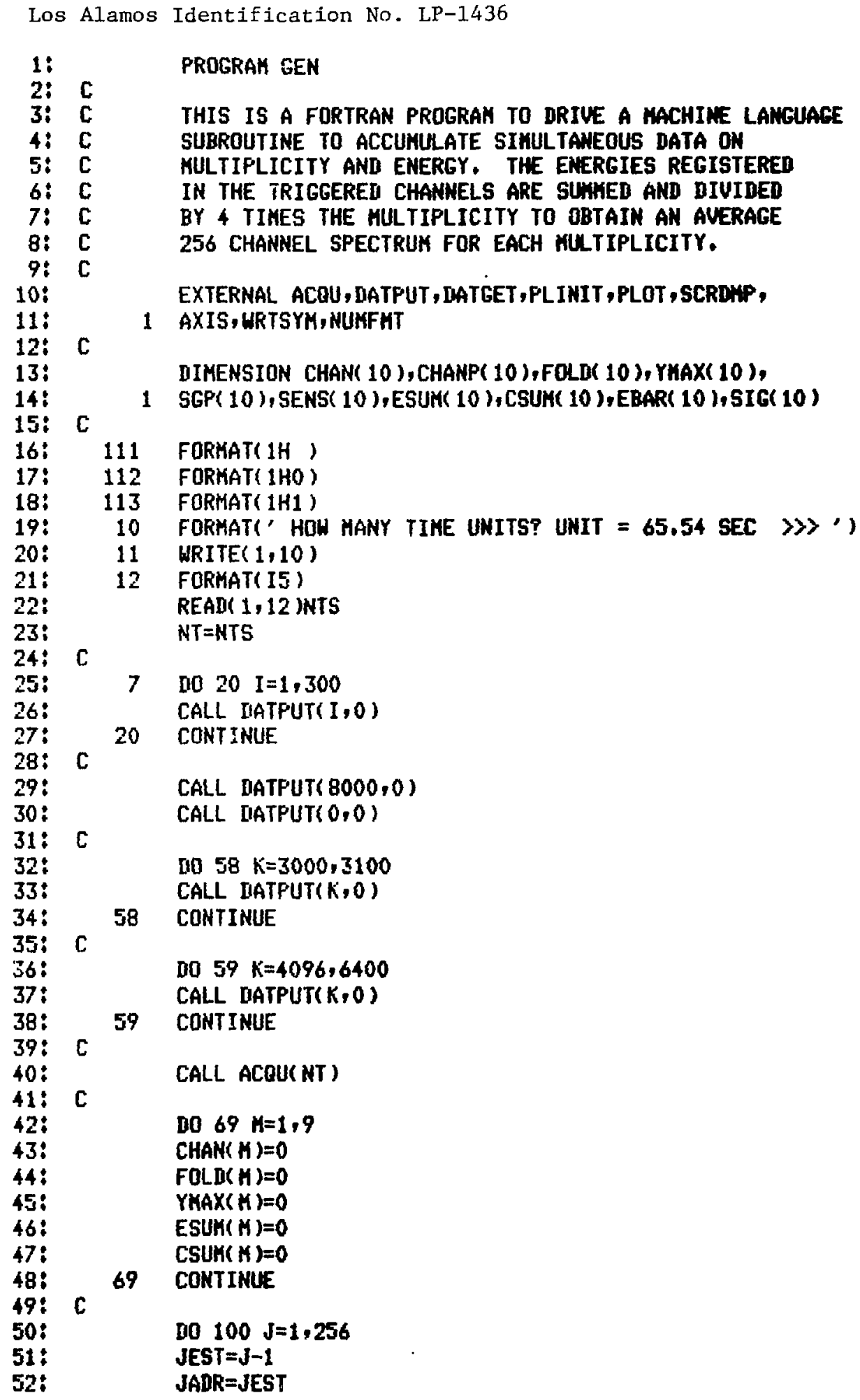




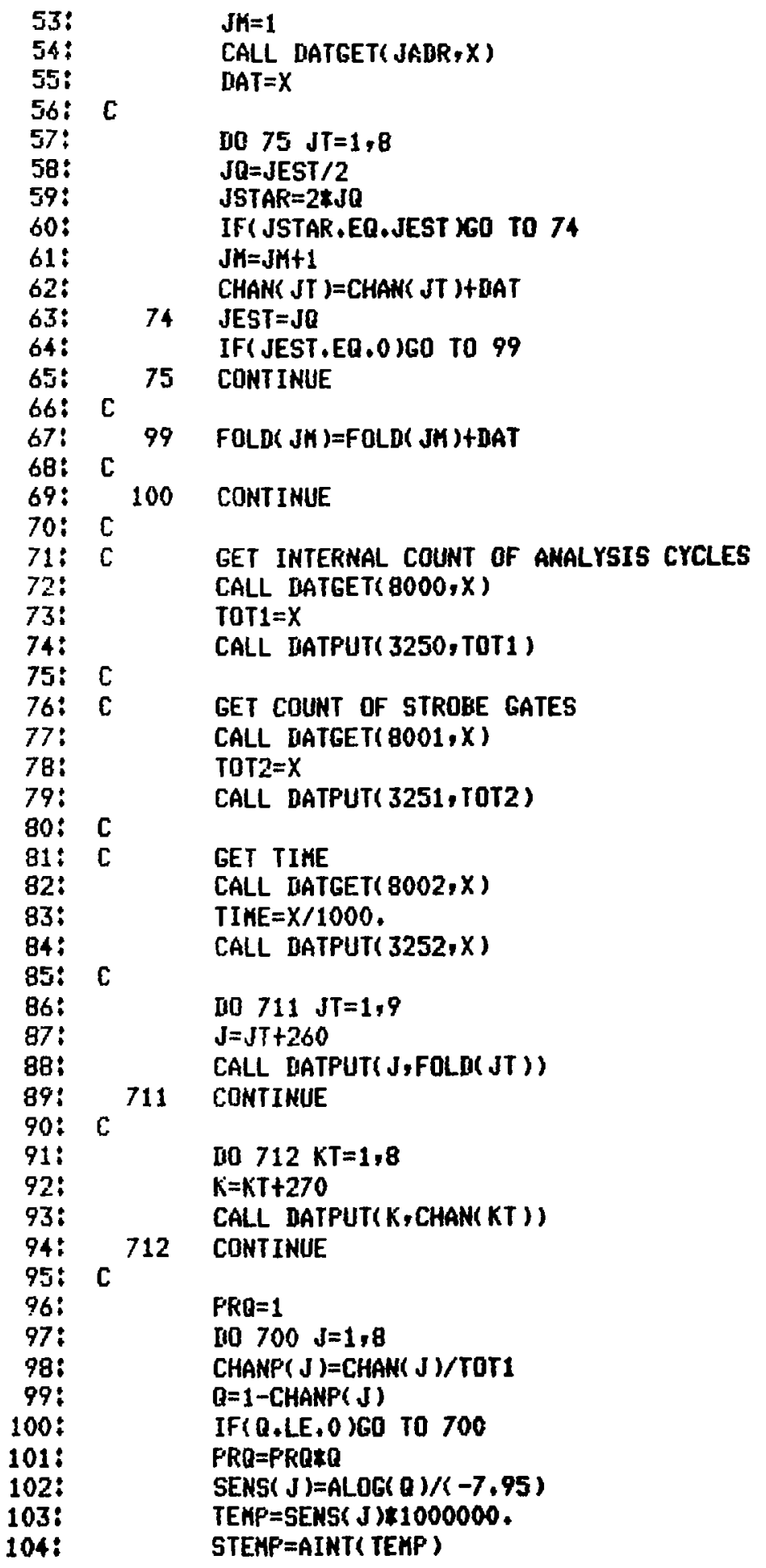




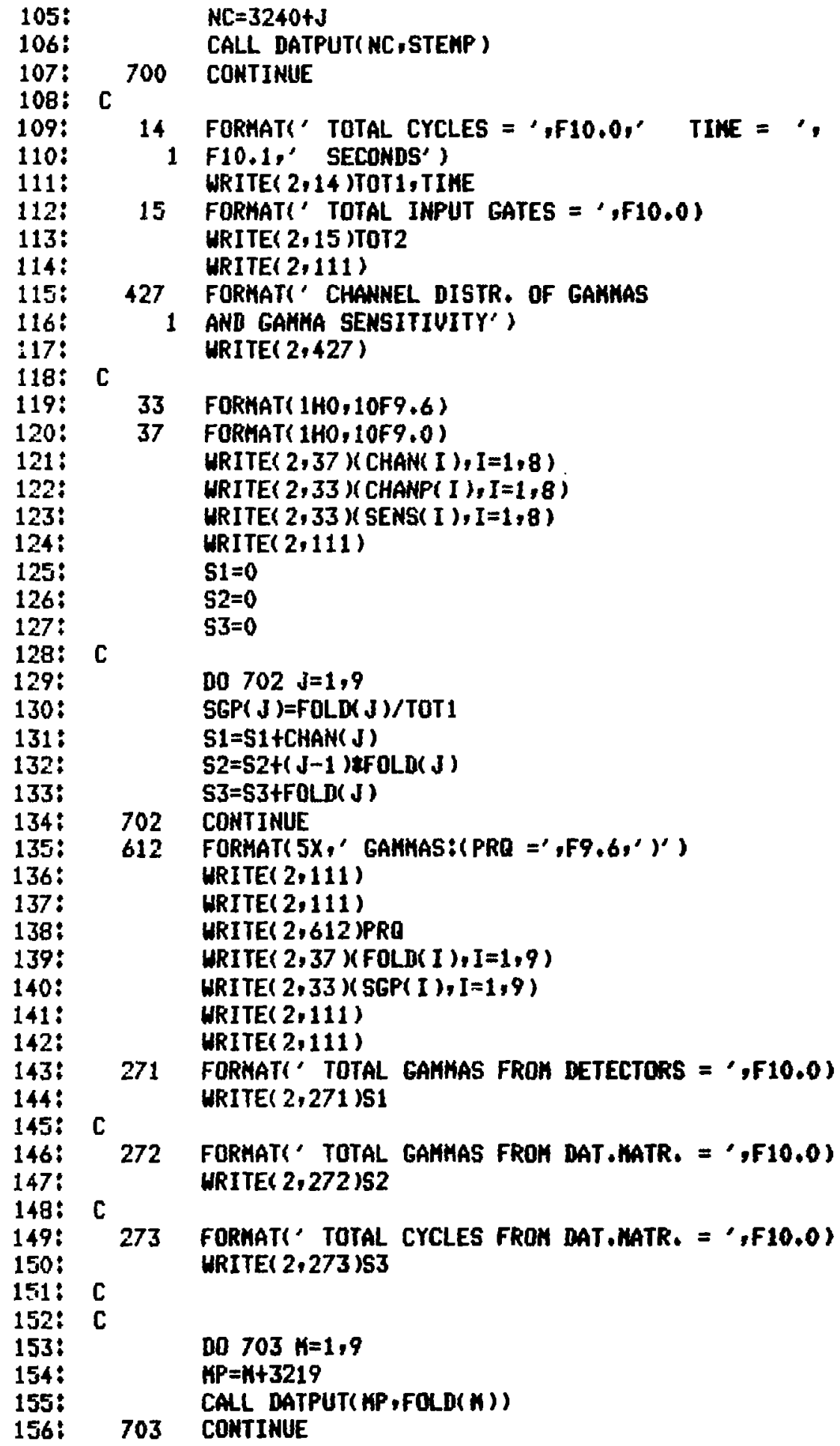




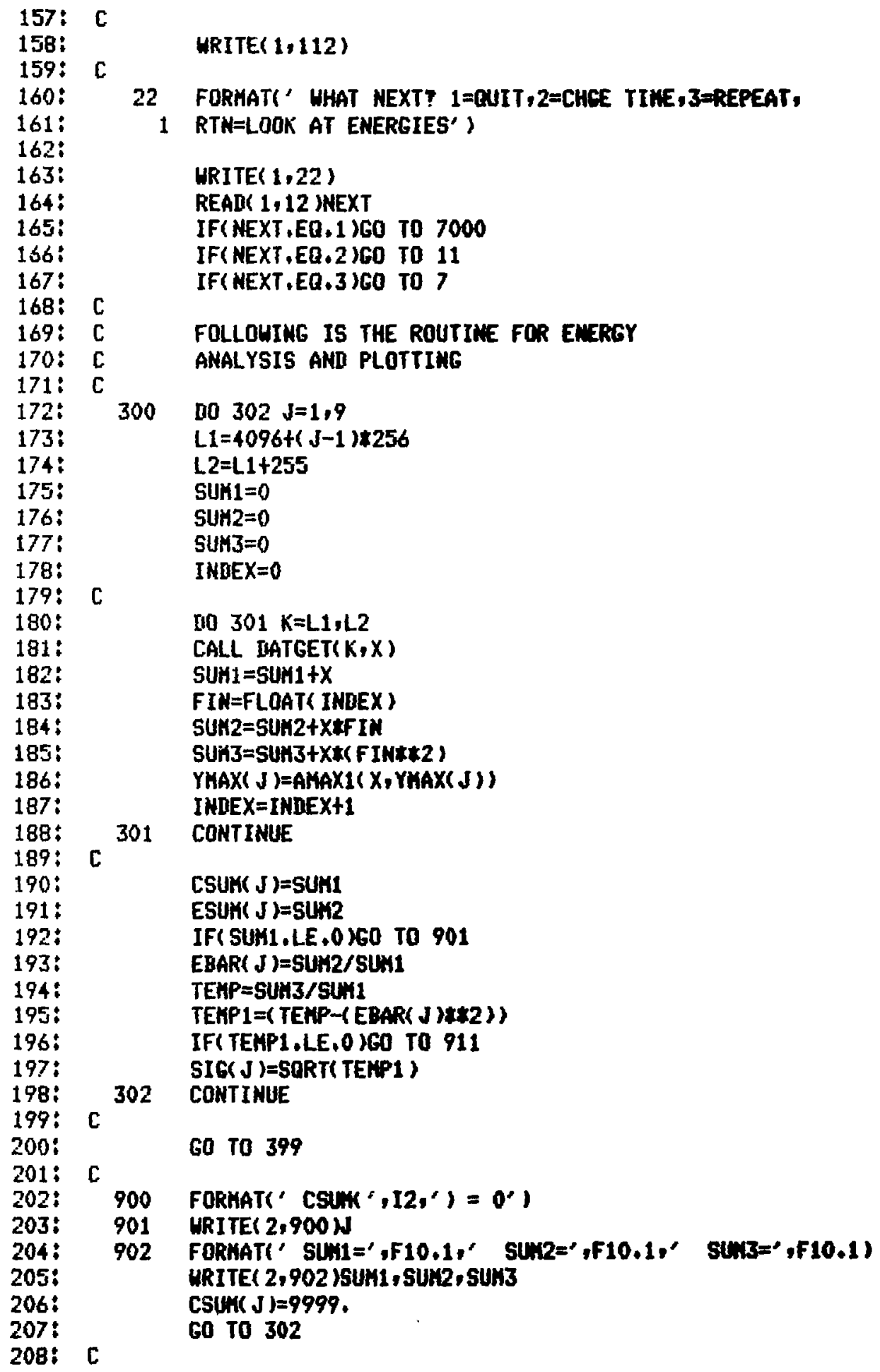




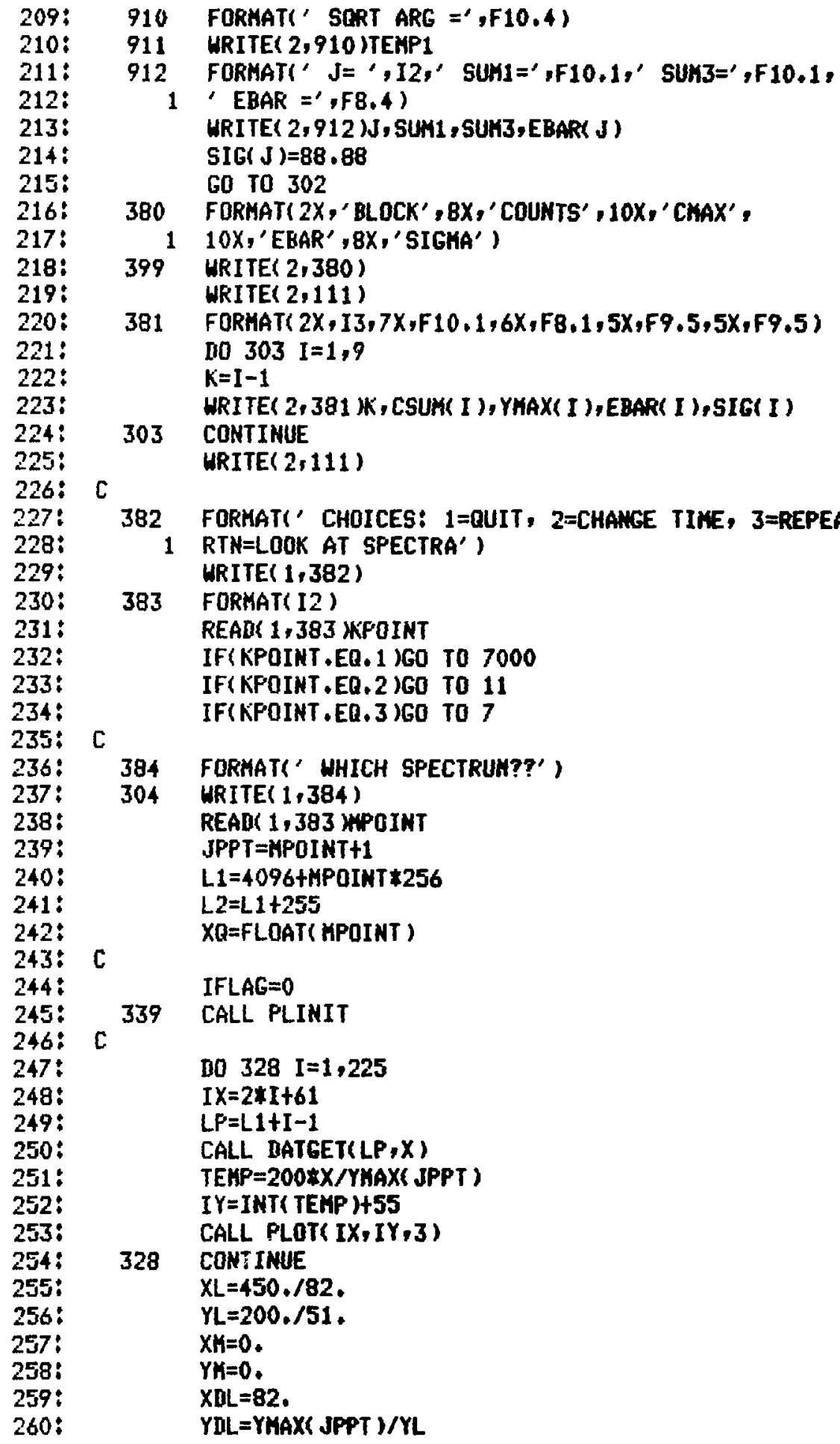




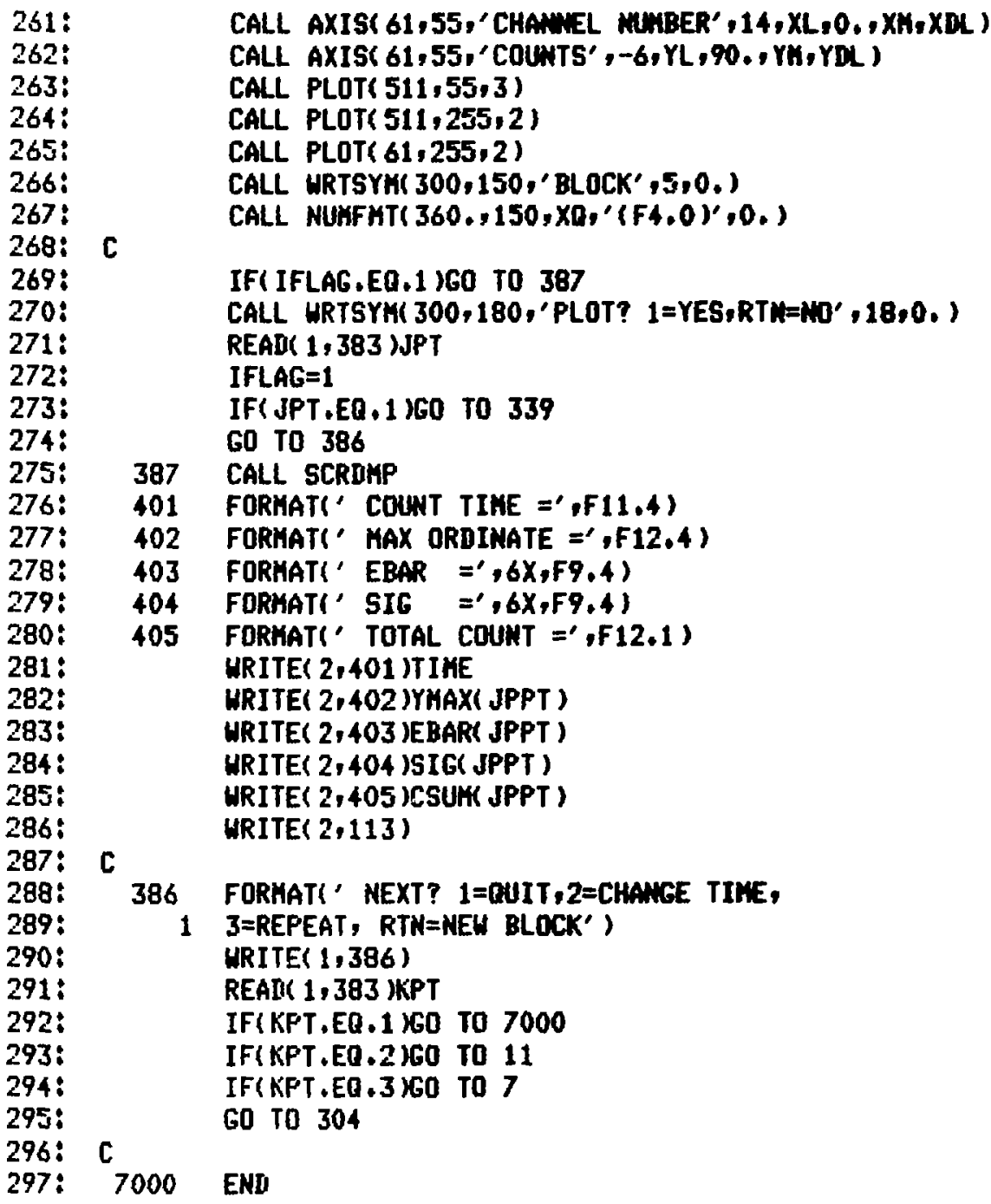




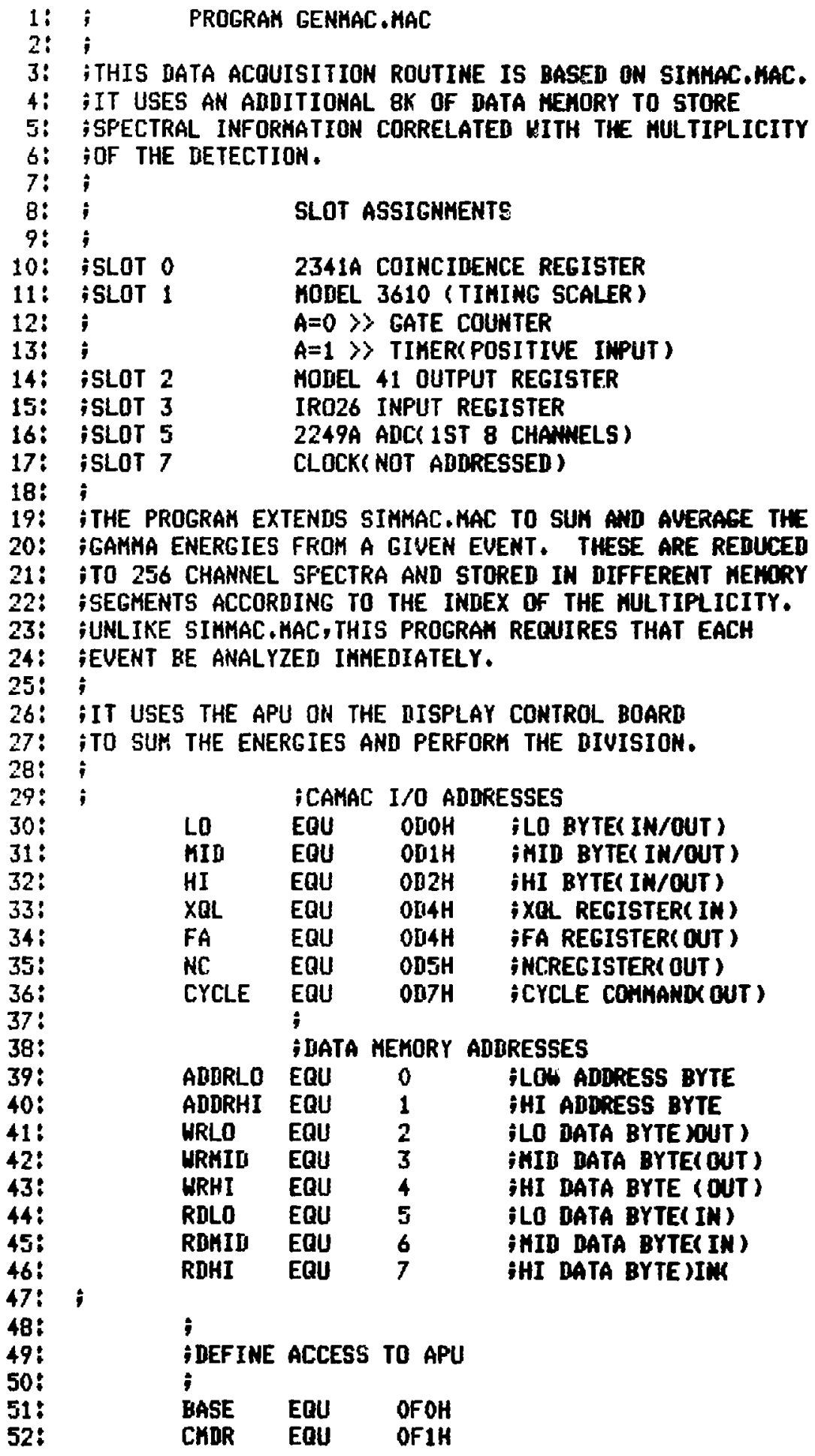




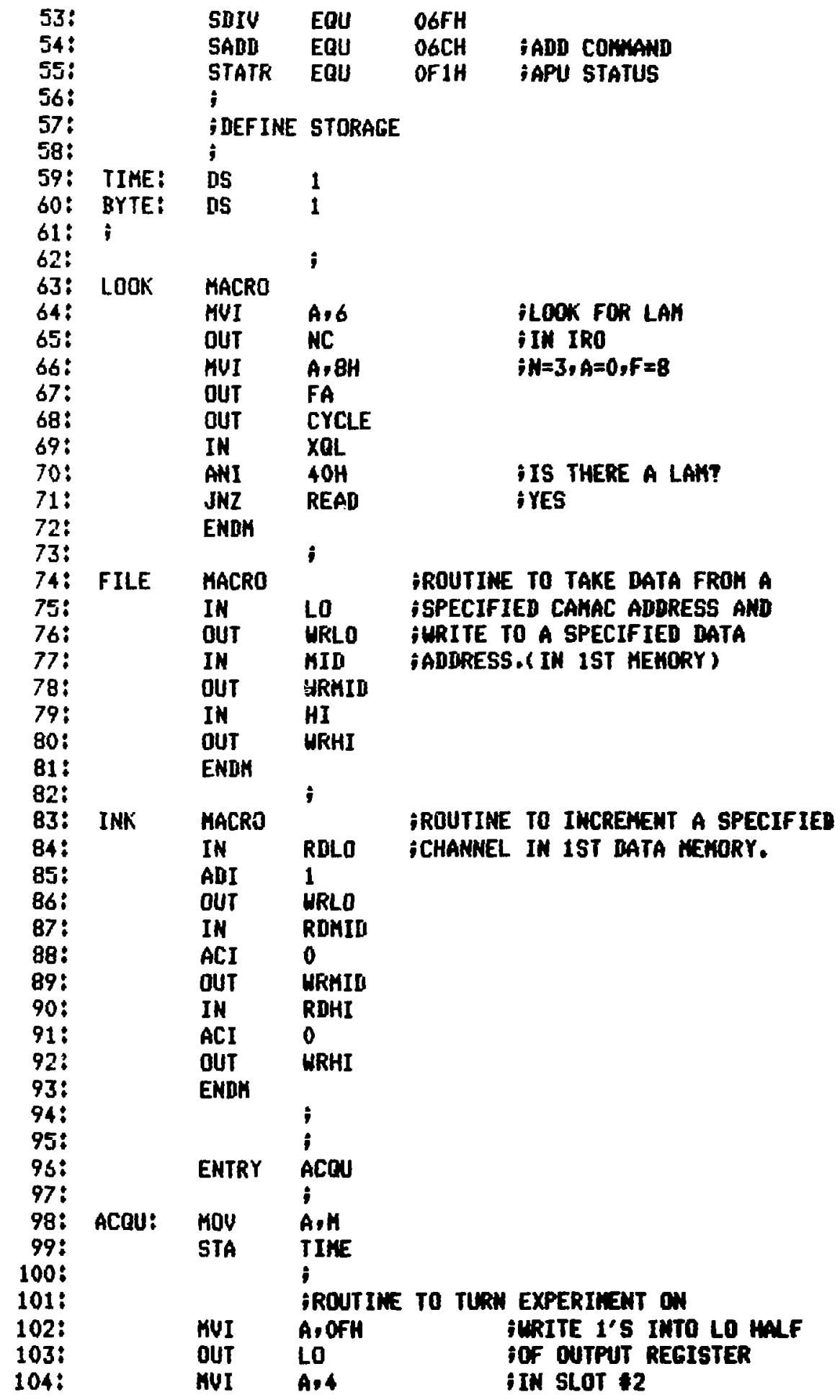




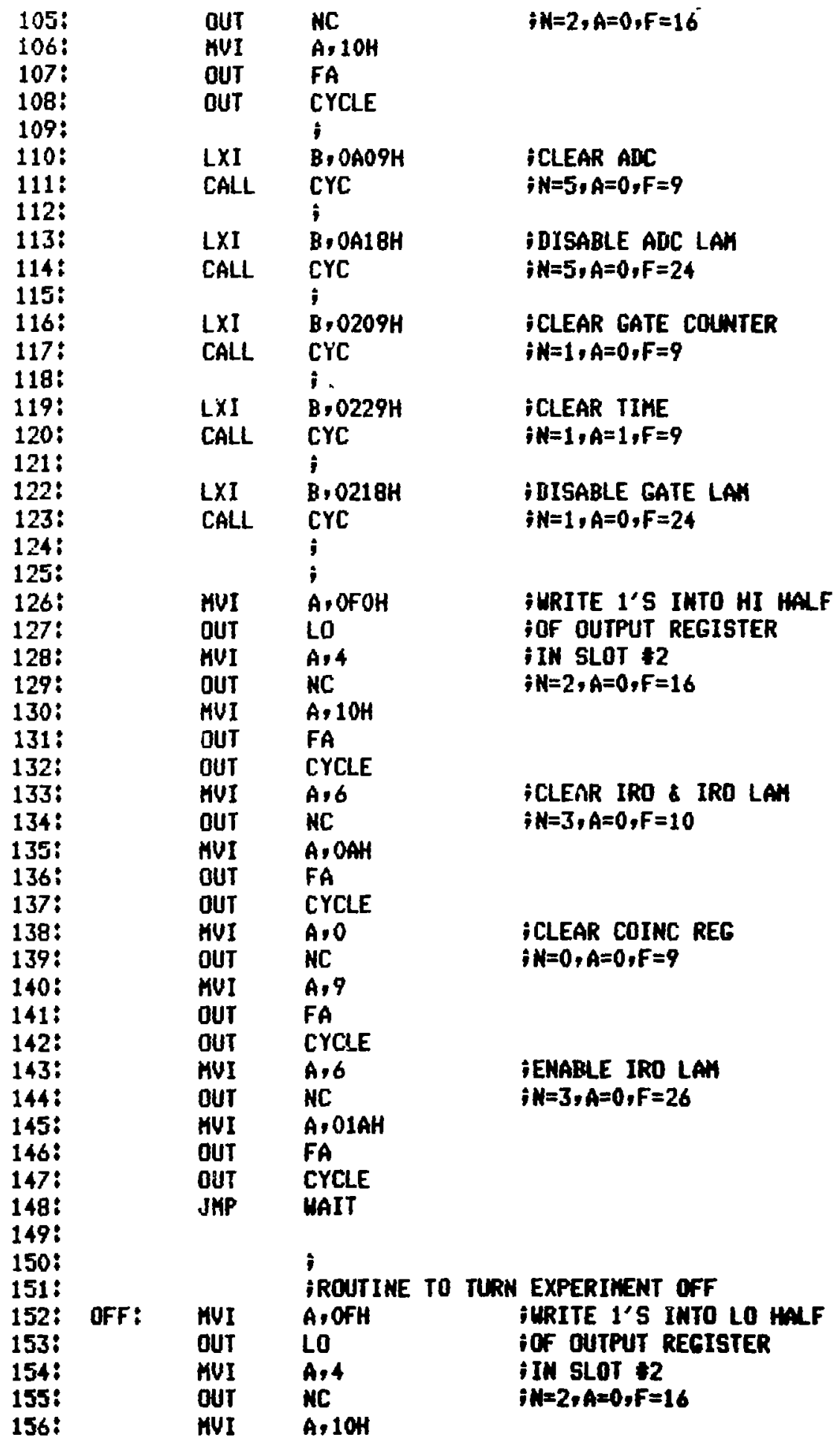




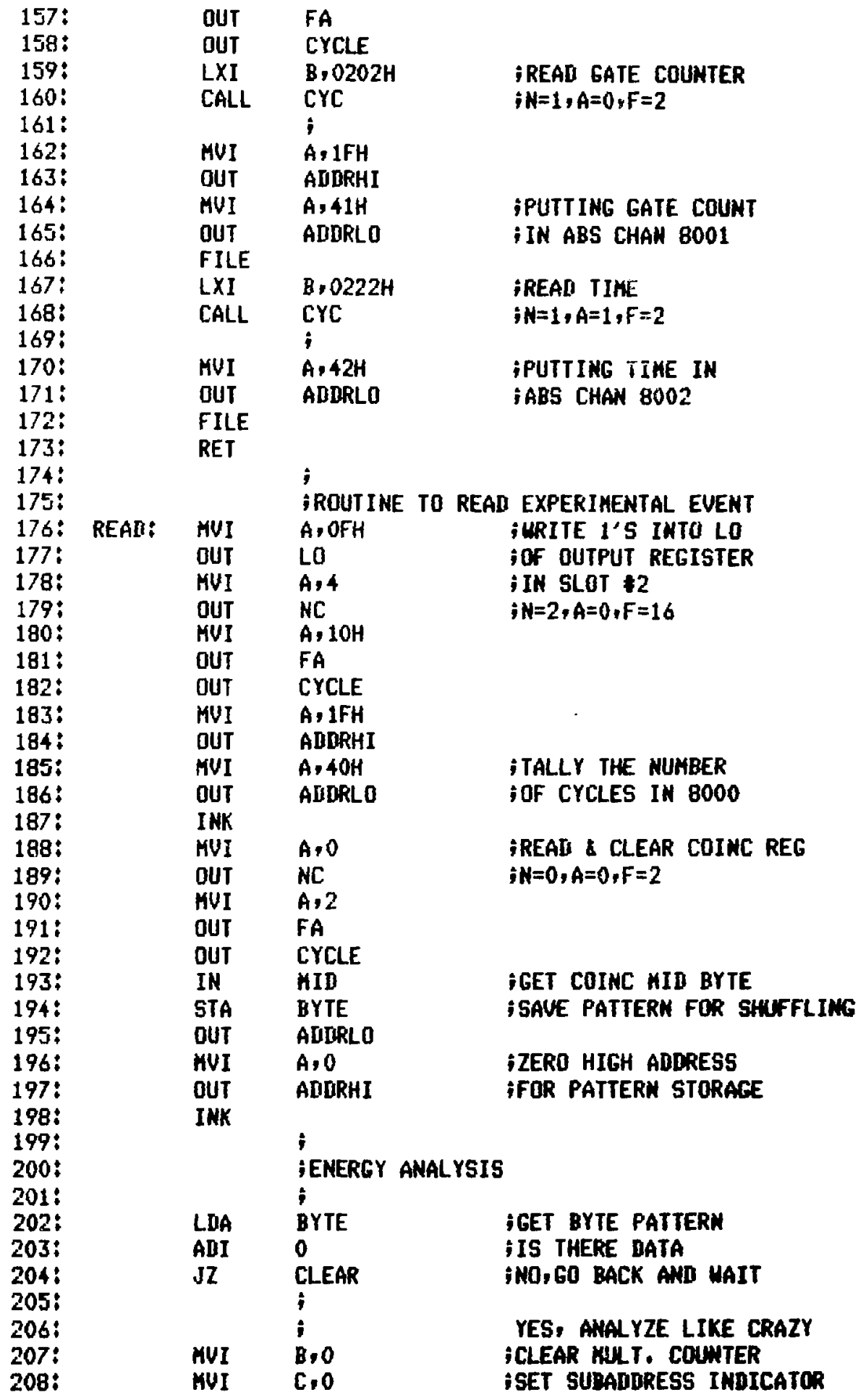




\begin{tabular}{|c|c|c|c|c|}
\hline $\begin{array}{l}209: \\
210:\end{array}$ & & $\begin{array}{l}\text { HUI } \\
\text { OUT }\end{array}$ & $\begin{array}{l}A, 0 \\
\text { BASE }\end{array}$ & \\
\hline $211 ;$ & & OUT & BASE & ;CLEAR APU TO ADD EMERGIES \\
\hline $212:$ & & RUI & 0,8 & ISET LOOP COUNTER \\
\hline 213: & & & $i$ & \\
\hline $\begin{array}{l}214 i \\
215 i\end{array}$ & LOOP: & $\begin{array}{l}\text { LDA } \\
\text { RAR }\end{array}$ & BYTE & ;GET BYTE PATTERK \\
\hline 216: & & STA & BYTE & \\
\hline $217 !$ & & JNC & AGAIN & \\
\hline 218: & & & ; & \\
\hline $217:$ & & INR & B & IYES, TALLY OME GAMMA \\
\hline $\begin{array}{l}220 ! \\
221:\end{array}$ & & $\begin{array}{l}\text { MCV } \\
\text { RRC }\end{array}$ & A,C & ;GET SUBADDRESS OF GAKHA \\
\hline $222:$ & & $\begin{array}{l}\text { RKL } \\
\text { RRC }\end{array}$ & & \\
\hline $223:$ & & RRC & & \\
\hline $224:$ & & ANI & $\mathrm{OEOH}$ & \\
\hline 225: & & OUT & FA & ;F $=0$, SO OUT TO FA \\
\hline 226: & & MUI & $A, O A H$ & $i N=5$ \\
\hline 227: & & OUT & $M C$ & \\
\hline $\begin{array}{l}228: \\
229:\end{array}$ & & OUT & CYCLE & iREAI IMDICATED HCA CHAMMEL \\
\hline $\begin{array}{l}229: \\
230 !\end{array}$ & & $\begin{array}{l}\text { In } \\
\text { DUT }\end{array}$ & BASE & ;PUT ADC DATA IN APU \\
\hline 231: & & IN & MID & ;FOR ADDING \\
\hline 232: & & OUT & BASE & \\
\hline 233: & & HVI & A, SADD & ;GET ADD COMHAND \\
\hline $\begin{array}{l}234: \\
235:\end{array}$ & & OUT & CMUR & 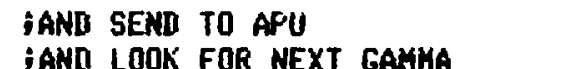 \\
\hline $236:$ & AGAIN: & INR & C & FADUANCE SUBADDRESS \\
\hline 237: & & DCR & & ¿CHECK OFF ONE ADC \\
\hline 238: & & $\mathrm{JZ}$ & CALC & IIF DOHE, CALC AUERAGE \\
\hline $\begin{array}{l}239: \\
240:\end{array}$ & & JHP & LOOP & IMOT DONE,LOOK FOR NEXT GAMMA \\
\hline 241: & CALC: & HOV & $A, B$ & ;GET MUKBER OF GAKMAS \\
\hline $242:$ & & RLC & & \\
\hline $\begin{array}{l}243: \\
244:\end{array}$ & & $\begin{array}{l}\text { RLC } \\
\text { ANI }\end{array}$ & & \\
\hline $\begin{array}{l}244: \\
245:\end{array}$ & & $\begin{array}{l}\text { ANI } \\
\text { OUT }\end{array}$ & $\begin{array}{l}\text { O3CH } \\
\text { BASE }\end{array}$ & $\begin{array}{l}\text {;KULT IPLY BY } 4 \\
\text {;LOH BYTE OF DIUISOR }\end{array}$ \\
\hline $246:$ & & KUI & $A: 0$ & \\
\hline $247 i$ & & OUT & BASE & ;HI BYTE OF DIVISOR \\
\hline 248: & & KUI & A.SDIV & ;GET DIVIDE COMAND \\
\hline $\begin{array}{l}249: \\
250:\end{array}$ & & OUT & CMDR & ;AND SEND TO APU \\
\hline $\begin{array}{l}250 ! \\
251: \\
252:\end{array}$ & & & & $\begin{array}{l}\text {;THIS GIUES AUERAGE ENERGY/4 } \\
\text {;GIUING A } 256 \text { CHANAEL SPECTRIM }\end{array}$ \\
\hline $\begin{array}{l}254: \\
255:\end{array}$ & & ADI & & \\
\hline $\begin{array}{l}255: \\
256:\end{array}$ & & JH & NTDN & \\
\hline 257 & & IN & $\begin{array}{l}\text { BASE } \\
\text { BASE }\end{array}$ & IAND USE LOU BYTE OF QUOTIENT \\
\hline 258: & & & & IFOR LOH ADDRESS \\
\hline $\begin{array}{l}259: \\
260:\end{array}$ & & $\begin{array}{l}\text { DUT } \\
\text { MUI }\end{array}$ & $\begin{array}{l}\text { ADDRLO } \\
\text { A,O1OH }\end{array}$ & \\
\hline
\end{tabular}




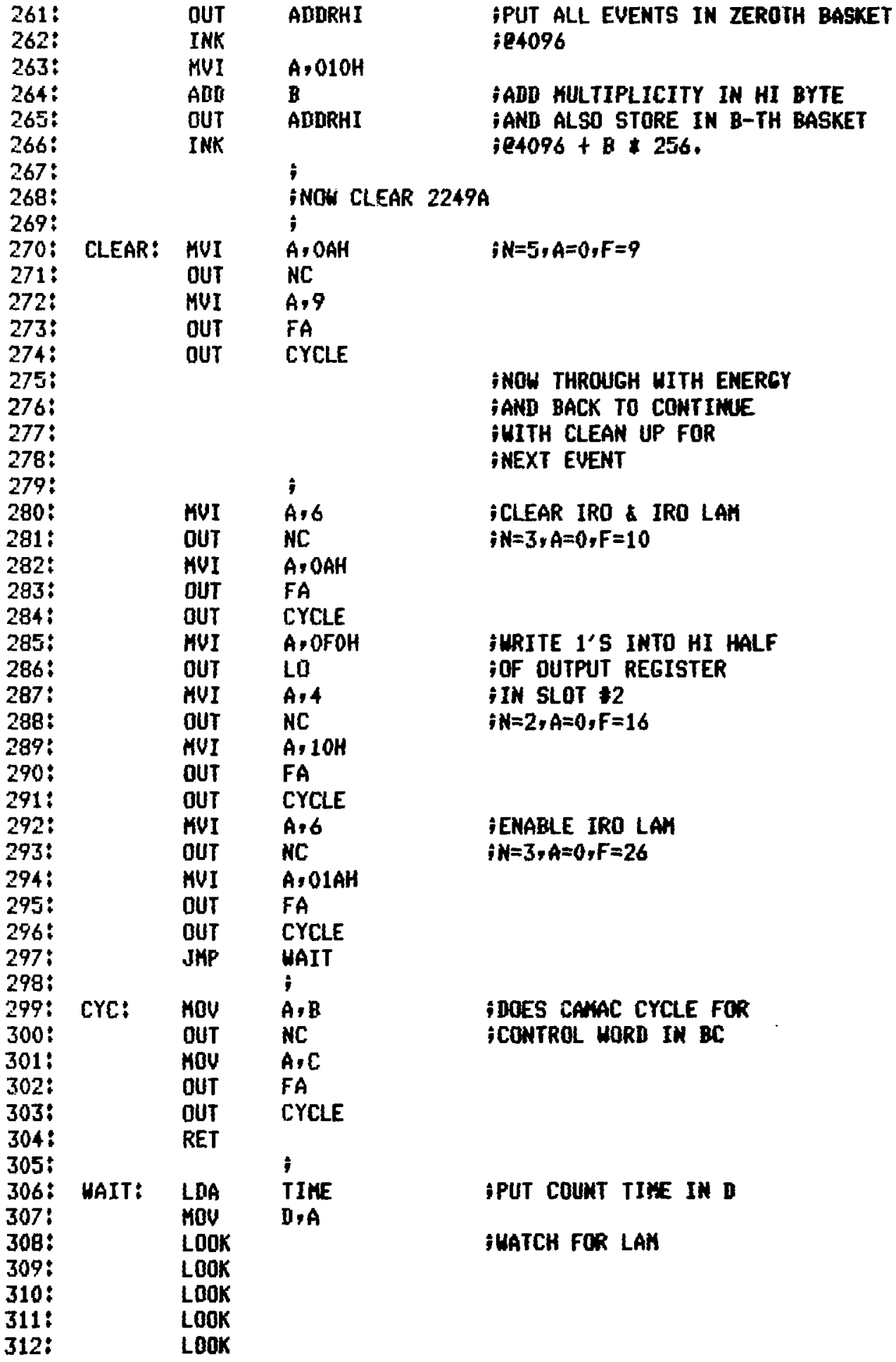




$\begin{array}{llll}313: & \text { HUI } & \text { A,O2H } & \text { iCHECK TIHE } \\ 314: & \text { OUT } & \text { NC } & \text { iREAD CLOCK } \\ 315: & \text { HUI } & A, 020 H & \text { iN=1,A=1,F=0 } \\ 316: & \text { OUT } & \text { FA } & \\ 317: & \text { OUT } & \text { CYCLE } & \text { iAND COMPARE } \\ 318: & \text { IN } & \text { HI } & \\ 319: & \text { CHP } & \text { D } & \text { IHITH TIHE IN D } \\ 320: & \text { JC } & \text { HAIT } & \text { iNOT OUT YET } \\ 321: & \text { JHP } & \text { OFF } & \text { iTIME IS OUT } \\ 322: & \text { END } & & \end{array}$




\section{ACKNOWLEDGMENTS}

I wish to acknowledge with gratitude the hospitality, interest, and assistance of the many Los Alamos people who contributed to this work. I thank William G. Davey, former $Q$ (Energy) Division Leader; and Carl Henry and E. J. Dowdy, former and present Group Leaders of Group Q-2 (Advanced Nuclear Technology) for their personal and official support. Special thanks go to Gordon Hansen for hours of counsel and encouragement, and for performing many supporting calculations. John Pratt contributed greatly in the procurement of equipment and in putting it in operation. Everyone in Q-2 contributed with a helping hand and a smile for a visitor.

I appreciate the courtesy of W. K. McFarlane and the Experimental Elementary Particle Physics Group of Temple University who loaned the high-performance photomultipliers and preamplifiers used in the main detector array. 
[ 1] EARL K. HYDE, Ed., The Nuclear Properties of the Heavy Elements, Vol. III, Chapt. 7, Prentice-Hall (1964).

[ 2] A. B. SMITH, P. A. FIELDS, and A. M. FRIEDMAN, Phys. Rev. 104, $699(1956)$.

[ 3] H. R. BOWMAN and S. G. THOMPSON, Proc. 2nd International Conference on Peaceful Uses of Atomic Energy (1958), Vol. 15, p. 212, United Nations, Geneva (1958).

[ 4] G. V. VAL'SKII, B. M. ALEKSANDROV, I. A. BARANOV, A. S. KRIVOKHATSKII, G. A. PETROV, and YU. S. PLEVA, Soviet Journal of Nuclear Physics, 10137 (1969).

[ 5] V. V. VERBINSKI, HANS WEBER, and R. E. SUND, Phys. Rev. C, 1, 1173 (1973).

[ 6] F. Pleasanton, Personal Communication (1974) to authors of following reference [Ref. 7].

[ 7] D. C. HOFFMAN and M. M. HOFFMAN, Annual Review of Nuclear Science, 24, 151-207 (1974).

[ 8] K. RUMPOLD, F. SEMTURS, P. WEINZIERI, Proc. IAEA Symposium on Physics and Chemistry of Fission, Vienna, Austria (1969), p. 519, International Atomic Energy Agency (1969).

[ 9] A. LAJTAI, L. JEKI, Gy. KLUGE, I. VINNARY, F. ENGARD, P. P. DYACHENKO, and B. D. KUZMINOV, Proc. IAEA Symposium on Physics and Chemistry of Fission, Rochester, New York (1973), Vol. II, p. 249, International Atomic Energy Agency (1974).

[10] K. SKARSVAG, Phys. Rev. C 22, 638 (1980).

[11] H. NIFENECKER, C. SIGNARBIEUX, M. RIBRAG, J. POITOU, and J. MATUSZEK, Nuclear Physies 1189,285 (1972).

[12] V. S. RAMAMURTHY, R. K. CHOUDHURY, and J. C. MOHANKRISHNA, Pramana (India), 8, 322 (1977).

[13] L. E. GLENDENIN, J. P. UNIK, and H. C. GRIFFIN, Proc. IAEA Symposium on Physics and Chemistry of Fission, p. 369 , Vol. I, Salzburg, Austria (1965), International Atomic Energy Agency (1965). 
[14] S. A. E. JOHANSSON and P. KLEINHEINZ, Alpha-Beta- and Gamma-Ray Spectrometry, KAI SIEGBAHN, Ed., North Holland Publishing Co., Amsterdam, Holland (1968).

[15] Nuclear Enterprises, Inc., San Carlos, California.

[16] Radio Corporation of America.

[17] C. M. LEDERER and V. S. SHIRLEY, Eds., Table of Isotopes, 7th ed., p. 1512, John Wiley \& Sons.

[18] Amperex Sales Corp., Slatersville, Rhode Island.

[19] Phillips Scientific, Suffern, New York.

[20] LeCroy Research Systems, Spring Valley, New York.

[21] E. NARDI, Nucl. Instr. and Meth., 95 (1979) 229.

[22] R. EVANS, The Atomic Nucleus, pp. 674-692, McGraw-Hill (1955).

[23] S. Y. VAN DER WERF, Nucl. Instr. and Meth., 153 (1978) 221.

[24] H. R. BOWMAN, S. G. THOMPSON, R. L. WATSON, S. S. KAPOOR, and J. O. RASMUSSEN, Proc. IAEA Symposium on Physics \& Chemistry of Fission, Salzburg, Austria (1965), Vol. II, p. 125, International Atomic Energy Agency (1965).

[25] S. Y. VAN DER WERF, Personal Communication, 1981. 